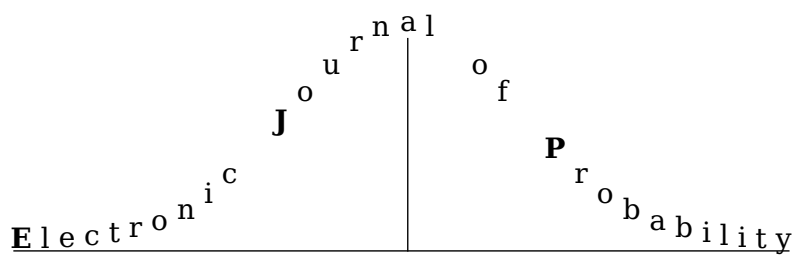

Electron. J. Probab. 24 (2019), no. 33, 1-38.

ISSN: 1083-6489 https://doi.org/10.1214/19-EJP299

\title{
Continuous-state branching processes with competition: duality and reflection at infinity
}

\author{
Clément Foucart*
}

\begin{abstract}
The boundary behavior of continuous-state branching processes with quadratic competition is studied in whole generality. We first observe that despite competition, explosion can occur for certain branching mechanisms. We obtain a necessary and sufficient condition for $\infty$ to be accessible in terms of the branching mechanism and the competition parameter $c>0$. We show that when $\infty$ is inaccessible, it is always an entrance boundary. In the case where $\infty$ is accessible, explosion can occur either by a single jump to $\infty$ (the process at $z$ jumps to $\infty$ at rate $\lambda z$ for some $\lambda>0$ ) or by accumulation of large jumps over finite intervals. We construct a natural extension of the minimal process and show that when $\infty$ is accessible and $0 \leq \frac{2 \lambda}{c}<1$, the extended process is reflected at $\infty$. In the case $\frac{2 \lambda}{c} \geq 1, \infty$ is an exit of the extended process. When the branching mechanism is not the Laplace exponent of a subordinator, we show that the process with reflection at $\infty$ gets extinct almost surely. Moreover absorption at 0 is almost sure if and only if Grey's condition is satisfied. When the branching mechanism is the Laplace exponent of a subordinator, necessary and sufficient conditions are given for a stationary distribution to exist. The Laplace transform of the latter is provided. The study is based on classical time-change arguments and on a new duality method relating logistic CSBPs with certain generalized Feller diffusions.
\end{abstract}

Keywords: continuous-state branching process; generalized Feller diffusion; branching process with interaction; explosion; coming down from infinity; entrance boundary; reflecting boundary; Lamperti's time change; duality.

AMS MSC 2010: 60J80; 60J70; 92D25.

Submitted to EJP on September 26, 2018, final version accepted on March 24, 2019.

Supersedes arXiv: 1711.06827.

\section{Introduction}

Continuous-state branching processes (CSBPs for short) have been defined by Jiřina [Jiř58] and Lamperti [Lam67a] for modelling the size of a random continuous population whose individuals reproduce and die independently with the same law. Lamperti

\footnotetext{
*Université Paris 13, Laboratoire Analyse, Géométrie \& Applications UMR 7539 Institut Galilée, France. E-mail: foucart@math. univ - paris13.fr
} 
[Lam67b] and Grimvall [Gri74] have shown that these processes arise as scaling limits of Galton-Watson Markov chains. Their laws are characterised in terms of a LévyKhintchine function $\Psi$ (called a branching mechanism). A shortcoming of CSBPs for modelling population lies in their degenerate longterm behavior. In the long run, a CSBP either tends to 0 or to $\infty$. On the event of extinction, the process can decay indefinitely or be absorbed at 0 in finite time. Similarly, on the event of non-extinction, the CSBP can grow indefinitely or be absorbed at $\infty$ in finite time. The latter event is called explosion and occurs typically when the process performs infinitely many large jumps in a finite time with positive probability.

Since the sixties, several generalizations of CSBPs have been defined to overcome various unrealistic properties of pure branching processes. Lambert [Lam05] has introduced a generalization of these processes by incorporating pairwise interactions between individuals. These processes, called logistic continuous-state branching processes, are the random analogues of the logistic equation

$$
\mathrm{d} z_{t}=\gamma z_{t} \mathrm{~d} t-\frac{c}{2} z_{t}^{2} \mathrm{~d} t
$$

where, informally speaking, the Malthusian growth $\gamma z_{t} \mathrm{~d} t$ is replaced by the full dynamics of a continuous-state branching process. For instance, when the mechanism $\Psi$ of the CSBP reduces to $\Psi(z)=\frac{\sigma^{2}}{2} z^{2}-\gamma z$, the process $\left(Z_{t}, t \geq 0\right)$ is the logistic Feller diffusion

$$
\mathrm{d} Z_{t}=\sigma \sqrt{Z_{t}} \mathrm{~d} B_{t}+\gamma Z_{t} \mathrm{~d} t-\frac{c}{2} Z_{t}^{2} \mathrm{~d} t .
$$

The negative quadratic drift represents additional deaths occurring due to pairwise fights among individuals. Intuitively, these fights can be interpreted as competition (for resources for instance). We refer to Le, Pardoux and Wakolbinger [LPW13] and Berestycki, Fittipaldi and Fontbona [BFF17] for a study of the competition at the level of the genealogy. In a logistic CSBP, individuals and their progenies are not independent of one another, and the branching property, from which all properties of CSBPs can be deduced, is lost. One of the main reasons for interest in logistic CSBPs is to provide a model of a population with possible self-limiting growth.

The objective of this article is to study these processes with most general mechanisms and to understand precisely how the competition regulates the growth. We shall study the nature of the boundaries 0 (extinction of the population) and $\infty$ (explosion of the population). Throughout this article, we follow the terminology of Feller, introduced in [Fel54], for classifying boundaries of a diffusion (see Section 2 for their meaning). The state of the art is as follows. In the continuous case (1.2), Feller tests provide that $\infty$ is an entrance and 0 an exit. For a general mechanism $\Psi$, the logistic CSBP has (typically unbounded) positive jumps and such general tests do not exist. Lambert in [Lam05] has found a set of sufficient conditions on the mechanism $\Psi$ for $\infty$ to be an entrance boundary. Under these conditions, it is also shown in [Lam05] that the competition alone has no impact on the extinction of the population. In other words, the boundary 0 is an exit if and only if the branching mechanism $\Psi$ satisfies Grey's condition. The entrance property from $\infty$ coincides with the notion of coming down from infinity observed in many stochastic models. We refer for instance to Cattiaux et al. [CCL ${ }^{+}$09] and Li [Li18] for recent related works and to Donnelly [Don91] for a classical result in coalescent theory.

We shall choose a different path than [Lam05] and study directly the semigroup of logistic CSBPs. A rather surprising first phenomenon is that the competition does not always prevent explosion. Some reproduction laws have large enough tails for $\infty$ to be accessible (meaning for explosion to occur). We provide a necessary and sufficient condition for $\infty$ to be inaccessible and show that under this condition the boundary $\infty$ is 
an entrance. Since the competition pressure increases with the size of the population, one may wonder if some compensation occurs near the boundary $\infty$ for a general mechanism $\Psi$. The main contribution of this article is to answer the following question. Is it possible for a logistic continuous-state branching process to leave and return to $\infty$ in finite time? We shall indeed see that the reproduction can be strong enough for explosion to occur and the quadratic competition strong enough to instantaneously push the population size back into $[0, \infty)$ after explosion. This phenomenon is captured by the notion of regular instantaneous reflecting boundary. By reflecting, we mean here that the Lebesgue measure of $\left\{t \geq 0, Z_{t}=\infty\right\}$ is zero almost surely. The boundary is instantaneous in the sense that starting from $\infty$ the process enters immediately $(0, \infty)$. Only in some cases, for which explosion is made by a single jump, the boundary $\infty$ is an exit. We stress also that it may well occur that the population goes extinct after exploding, so that $\infty$ is not always recurrent.

In order to classify the boundaries as explained above, we need to define an extension of the minimal process in $[0, \infty]$. This requires in general a deep study of the minimal semi-group. However, since processes with competition do not satisfy the branching property, most arguments for CSBPs are not applicable. The resolvent of logistic CSBPs is rather involved and we will not discuss all possibilities of extensions in this article but only construct a natural one by approximation. We first establish a duality between nonexplosive logistic continuous-state branching processes and some generalizations of the logistic Feller diffusion process (1.2). Namely we will show that when $\infty$ is inaccessible for the process $\left(Z_{t}, t \geq 0\right)$, for any $x \geq 0, z \in[0, \infty)$ and $t \geq 0$,

$$
\mathbb{E}_{z}\left(e^{-x Z_{t}}\right)=\mathbb{E}_{x}\left(e^{-z U_{t}}\right)
$$

where $\left(U_{t}, t \geq 0\right)$ is a solution to

$$
\mathrm{d} U_{t}=\sqrt{c U_{t}} \mathrm{~d} B_{t}-\Psi\left(U_{t}\right) \mathrm{d} t, \quad U_{0}=x .
$$

We shall see that the condition for $\infty$ to be inaccessible for $\left(Z_{t}, t \geq 0\right)$ is precisely given by Feller's test for 0 to be an exit of $\left(U_{t}, t \geq 0\right)$. We stress that the SDE above does not always have a unique solution as 0 can be regular for certain non-Lipschitz mechanisms $\Psi$. It is precisely for such mechanisms that $\infty$ will be regular for logistic CSBPs. Heuristically, if (1.3) holds for some processes $\left(Z_{t}, t \geq 0\right)$ and $\left(U_{t}, t \geq 0\right)$, then the entrance boundaries of $\left(Z_{t}, t \geq 0\right)$ will be classified in terms of exit boundaries of $\left(U_{t}, t \geq 0\right)$. We refer to Cox and Rösler [CR84] and Liggett [Lig05] for a study of boundaries by duality of semi-groups. The identity (1.3) provides a representation of the semi-group of any non-explosive process with competition and will allow us to construct an extended process over $[0, \infty]$ with $\infty$ reflecting as a limit of non-explosive processes. We highlight that this construction is different from the classical Itô's concatenation procedure for building recurrent extensions. In particular, our approach is not based on a measure theoretical description of the excursions from $\infty$ but on a direct description of the extended semi-group.

A very similar phenomenon of reflection at $\infty$ has been recently observed by Kyprianou et al. [KPRS17] for a certain exchangeable fragmentation-coalescence process (EFC process). We shall observe the same phase transition between the reflecting boundary case and the exit boundary one. We highlight that contrary to the process studied in [KPRS17], a logistic CSBP can reach $\infty$ by accumulation of large jumps over a finite interval of time. Discrete logistic branching processes are closely related to the number of fragments in some EFC processes, see Berestycki [Ber04] and Lambert [Lam05]. We refer also to Bertoin and Kortchemski [BK16, Section 5.3].

The duality (1.3) has been observed in a spatial context for the branching mechanism $\Psi(u)=\frac{\sigma^{2}}{2} u^{2}-\gamma u$ by Horridge and Tribe [HT04] for the logistic SPDE, see also 
Hutzenthaler and Wakolbinger [HW07]. Lastly, other competition mechanisms than the quadratic drift have been studied. We refer for instance to the monograph of Pardoux [Par16] and Ba and Pardoux [BP15] for some generalisations of the logistic Feller diffusions. It is worth noticing that the relation (1.3) does not hold for general competition mechanims.

The paper is organised as follows. In Section 2, we recall some known facts about CSBPs and define minimal logistic CSBPs through a martingale problem. We state our main results in Section 3 and provide some examples. In Section 4, we show how to solve the martingale problem by time-changing an Ornstein-Uhlenbeck type process. Some first properties of the minimal process, such as a criterion for its explosion, are derived from this time-change. In Section 5 , we gather the possible behaviors of the diffusion $\left(U_{t}, t \geq 0\right)$ at its boundaries. Then, in Section 6, we deal with the entrance property at $\infty$ of non-explosive logistic CSBPs. Explosive ones are studied in Sectio 7, where we define and study an extension of the minimal process with $\infty$ either regular or exit. Lastly, in the Appendix, we provide the calculations needed for classifying the boundaries of $\left(U_{t}, t \geq 0\right)$ according to $\Psi$ and the parameter $c$.

\section{Preliminaries}

As we will use Feller's terminology repeatedly, we briefly recall how to classify boundaries. Consider a process valued in an interval $[a, b]$ with $a, b \in \overline{\mathbb{R}}$ and $a<b$,

- the boundary $b$ is said to be accessible if there is a positive probability that it will be reached in finite time (the process can enter into $b$ ). If $b$ is accessible, then either the process cannot get out from $b$ and the boundary $b$ is said to be an exit or the process can get out from $b$ and the boundary $b$ is called a regular boundary.

- If the boundary $b$ is inaccessible, then either the process cannot get out from $b$, and the boundary $b$ is said to be natural or the process can get out from $b$ and the boundary $b$ is said to be an entrance.

Notation. We denote by $[0, \infty]$ the extended half-line and by $C_{b}([0, \infty])$ to be the space of continuous real-valued functions defined over $[0, \infty]$. Since $[0, \infty]$ is compact, any function $f \in C_{b}([0, \infty])$ is bounded. We set $\mathcal{D}([0, \infty])$ the space of càdlàg functions from $\mathbb{R}_{+}$to $[0, \infty]$. For any interval $I \subset \mathbb{R}$, we denote by $C_{c}^{2}(I)$ the space of continuous functions over $I$ with compact support that have continuous first two derivatives.

We recall the definition and some basic properties of continuous-state branching processes without competition. Most of the sequel can be found in Chapter 12 of Kyprianou's book [Kyp14]. A CSBP is a Feller process $\left(X_{t}, t \geq 0\right)$ valued in $[0, \infty]$ satisfying the branching property: for any $z, z^{\prime} \geq 0, t \geq 0$ and $x>0$

$$
\mathbb{E}_{z+z^{\prime}}\left[e^{-x X_{t}}\right]=\mathbb{E}_{z}\left[e^{-x X_{t}}\right] \mathbb{E}_{z^{\prime}}\left[e^{-x X_{t}}\right]
$$

The branching and Markov properties ensure the existence of a map $(x, t) \mapsto u_{t}(x)$ such that for all $x>0$ and all $t, s \geq 0, u_{t}(x) \in(0, \infty)$,

$$
\mathbb{E}_{z}\left[e^{-x X_{t}}\right]=e^{-z u_{t}(x)} \text { and } u_{s+t}(x)=u_{s} \circ u_{t}(x) .
$$

Silverstein in [Sil68, Theorem 4, page 1046] has shown that the map $t \mapsto u_{t}(x)$ is the unique solution to a non-linear ordinary differential equation

$$
\frac{\mathrm{d}}{\mathrm{d} t} u_{t}(x)=-\Psi\left(u_{t}(x)\right) \text { for all } x \in(0, \infty)
$$

where $\Psi$ is a Lévy-Khintchine function of the form

$$
\Psi(z)=-\lambda+\frac{\sigma^{2}}{2} z^{2}+\gamma z+\int_{0}^{+\infty}\left(e^{-z x}-1+z x \mathbb{1}_{\{x \leq 1\}}\right) \pi(\mathrm{d} x)
$$


with $\lambda \geq 0, \gamma \in \mathbb{R}, \sigma \geq 0$, and $\pi$ a Borel measure supported on $\mathbb{R}_{+}$satisfying

$$
\int_{0}^{+\infty}\left(1 \wedge x^{2}\right) \pi(\mathrm{d} x)<+\infty .
$$

Note that $\Psi(z)=\log \mathbb{E}\left[e^{-z Y_{1}}\right]$ for any $z \geq 0$, where $\left(Y_{t}, t \geq 0\right)$ is a spectrally positive Lévy process sent to $\infty$ at rate $\lambda \geq 0$. The function $\Psi$ is thus convex, infinitely differentiable on $(0, \infty)$, with $\Psi(0)=-\lambda$ and $\Psi^{\prime}(0+) \in[-\infty, \infty)$. Further, $-\Psi$ is the Laplace exponent of subordinator if and only if for any $z>0, \Psi(z)<0$. In such a case, $\Psi$ has the form

$$
\Psi(z)=-\lambda-\delta z-\int_{0}^{\infty}\left(1-e^{-z x}\right) \pi(\mathrm{d} x)
$$

where $\int_{0}^{\infty}(1 \wedge x) \pi(\mathrm{d} x)<\infty$ and $\delta \geq 0$ is a drift parameter. We refer for instance to [Kyp14, Section 8.1] for these properties. Any branching mechanism is Lipschitz on compact subsets of $(0, \infty)$ and thus the deterministic equation (2.2) admits a unique solution.

As in [Sil68], we interpret the killing term with parameter $\lambda$ as the possibility for the CSBP to jump to $\infty$ in finite time. Since for any $t \geq 0$ and any $x \in(0, \infty), u_{t}(x) \in(0, \infty)$, according to the semi-group equation (2.1), $\infty$ and 0 are either natural or exit boundaries. Grey [Gre74] classifies further the boundaries $\infty$ and 0 of a CSBP as follows.

- The boundary $\infty$ is accessible if and only if $\int_{0+} \frac{\mathrm{d} u}{|\Psi(u)|}<+\infty$.

- The boundary 0 is accessible if and only if $\int^{\infty} \frac{\mathrm{d} u}{|\Psi(u)|}<\infty$ (Grey's condition).

The integral conditions above ensure respectively the existence of a non-degenerate solution of (2.2) started from $x=0+$ and $x=\infty$. It is important to note that $\lambda=0$ is necessary for $\infty$ to be inaccessible but not sufficient. Indeed, the process can explode continuously by having unbounded paths over finite time intervals. A basic example is provided by the stable mechanism $\Psi(z)=-z^{\alpha}$ for $\alpha \in(0,1)$ which satisfies $\int_{0+} \frac{d u}{|\Psi(u)|}<$ $\infty$.

We now recall the long-term behavior of CSBPs. We refer to Theorem 12.5 in [Kyp14] for the following classification. Denote by $\rho$ the largest positive zero of $\Psi$, namely, $\rho:=\inf \{x>0, \Psi(x) \geq 0\}$, with the convention $\inf \emptyset=\infty$. For any $z \in[0, \infty]$,

$$
\mathbb{P}_{z}\left(X_{t} \underset{t \rightarrow \infty}{\longrightarrow} 0\right)=e^{-z \rho} \text { and } \mathbb{P}_{z}\left(X_{t} \underset{t \rightarrow \infty}{\longrightarrow} \infty\right)=1-e^{-z \rho} .
$$

When $-\Psi$ is the Laplace exponent of a subordinator, $\rho=\infty$ and the process goes to $\infty$ almost surely. Note also that there is a constant $\mathbf{d}>0$ such that for any large enough $u,-\frac{\Psi(u)}{u} \leq \mathbf{d}$ and thus $\int^{\infty} \frac{\mathrm{d} u}{|\Psi(u)|}=\infty$. When $-\Psi$ is not the Laplace exponent of a subordinator, $\Psi(u)>0$ for any large enough $u, \rho<\infty$ and the process goes to 0 with positive probability. If moreover $\int^{\infty} \frac{\mathrm{d} u}{\Psi(u)}=\infty$ then $X_{t} \underset{t \rightarrow \infty}{\longrightarrow} 0$ with positive probability albeit $X_{t}>0$ for all $t \geq 0$ almost surely. In the latter case, we say that 0 is attracting.

A classical construction of a CSBP with mechanism $\Psi$ is by time-changing the spectrally positive Lévy process $\left(Y_{t}, t \geq 0\right)$ (whose Laplace exponent is $-\Psi$ ) with the inverse of an additive functional. See for instance Lamperti [Lam67a], Caballero, Lambert and Uribe-Bravo [CLUB09]. In particular, the sample paths of a càdlàg CSBP have no negative jumps and are non-decreasing when $-\Psi$ is the Laplace exponent of a subordinator. This time-change leads to the following form for the generator of $\left(X_{t}, t \geq 0\right)$. For any $f \in C_{c}^{2}((0, \infty))^{1}$

$$
\mathscr{G} f(z):=-\lambda z f(z)+\frac{\sigma^{2}}{2} z f^{\prime \prime}(z)-\gamma z f^{\prime}(z)+z \int_{0}^{\infty}\left(f(z+u)-f(z)-u 1_{[0,1]}(u) f^{\prime}(z)\right) \pi(\mathrm{d} u) .
$$

\footnotetext{
${ }^{1}$ the space of twice continuously differentiable functions vanishing outside a compact subset of $(0, \infty)$.
} 
To incorporate quadratic competition, one considers an additional negative quadratic drift in the generator above and set

$$
\mathscr{L} f(z):=\mathscr{G} f(z)-\frac{c}{2} z^{2} f^{\prime}(z) .
$$

Definition 2.1. A minimal logistic continuous-state branching process is a càdlàg Markov process $\left(Z_{t}^{\min }, t \geq 0\right)$ on $[0, \infty]$ with 0 and $\infty$ absorbing, satisfying the following martingale problem (MP). For any function $f \in C_{c}^{2}((0, \infty))$, the process

$$
t \in[0, \zeta) \mapsto f\left(Z_{t}^{\min }\right)-\int_{0}^{t} \mathscr{L} f\left(Z_{s}^{\min }\right) \mathrm{d} s
$$

is a martingale, with $\zeta:=\inf \left\{t \geq 0 ; Z_{t}^{\min } \notin(0, \infty)\right\}$.

By definition the minimal process remains at the boundary once it has reached it. In particular, by setting $\zeta_{\infty}:=\inf \left\{t \geq 0, Z_{t}^{\min }=\infty\right\}$, on the event $\left\{\zeta=\zeta_{\infty}\right\}$, we have that $Z_{t}^{\min }=\infty$ for any $t \geq \zeta_{\infty}$. As already observed by Lambert [Lam05], one way to construct a minimal logistic CSBP is by time-changing an Ornstein-Uhlenbeck type process. The problem of explosion is not discussed in [Lam05] and we shall give out some details in Section 4.

In the sequel, we say that a Markov process $\left(Z_{t}, t \geq 0\right)$ extends the minimal process if $\left(Z_{t}, t \geq 0\right)$ takes its values in $[0, \infty]$ and $\left(Z_{t \wedge \zeta_{\infty}}, t \geq 0\right) \stackrel{\mathcal{L}}{=}\left(Z_{t}^{\min }, t \geq 0\right)$. Note that elementary return processes restarting after explosion from states in $(0, \infty)$ are ruled out from our study. We will only be interested in the existence of a continuous extension $\left(Z_{t}, t \geq 0\right)$, for which $Z_{t} \longrightarrow \infty$, almost surely, as $t \rightarrow \zeta_{\infty}+$. Moreover, the boundary $\infty$ will be said to be instantaneous if $\mathbb{P}_{\infty}(T=0)=1$ with $T:=\inf \left\{t>0, Z_{t}<\infty\right\}$ and reflecting, if $\mathbb{P}_{\infty}\left(Z_{t}=\infty\right)=0$ for any $t>0$, (equivalently the set $\left\{t>0, Z_{t}=\infty\right\}$ has a Lebesgue measure zero).

As explained in the introduction, the semi-group of logistic CSBPs will be represented in terms of a certain diffusion. For any mechanism $\Psi$ of the form (2.3), we call $\Psi$ generalized Feller diffusion, the minimal diffusion $\left(U_{t}, t<\tau\right)$ solving

$$
\mathrm{d} U_{t}=\sqrt{c U_{t}} \mathrm{~d} B_{t}-\Psi\left(U_{t}\right) \mathrm{d} t, \quad U_{0}=x
$$

where $\left(B_{t}, t \geq 0\right)$ is a Brownian motion and $\tau:=\inf \left\{t ; U_{t} \notin(0, \infty)\right\}$. As $u \mapsto \sqrt{u}$ is $1 / 2$ Hölder and $\Psi$ is locally Lipschitz, standard results (see e.g. [RY99, Section 3, Chapter IX]) ensure the existence and uniqueness of a strong solution to Equation (2.7) up to time $\tau$. Note that when $c=0$, the SDE (2.7) coincides with the ODE (2.2) and that (1.3) coincides with (2.1).

\section{Main results}

Theorem 3.1 (Accessibility of $\infty$ ). Assume $c>0$. The boundary $\infty$ is inaccessible for $\left(Z_{t}^{\min }, t \geq 0\right)$ if and only if

$$
\mathcal{E}:=\int_{0}^{\theta} \frac{1}{x} \exp \left(\frac{2}{c} \int_{x}^{\theta} \frac{\Psi(u)}{u} \mathrm{~d} u\right) \mathrm{d} x=\infty, \text { for some (and then for all) } \theta>0 .
$$

Remark 3.2. Note that when $\lambda>0, \int_{0}^{\theta} x^{\frac{2 \lambda}{c}-1} \mathrm{~d} x<\infty, \Psi(u) \underset{u \rightarrow 0+}{\longrightarrow} \Psi(0+)=-\lambda$ and thus $\mathcal{E}<\infty$. We shall see however that $\lambda>0$ is not necessary for $\infty$ to be accessible.

Theorem 3.1 is established in Section 4 . The next three theorems introduce extensions in $[0, \infty]$ of the minimal process. The usual convention $0 \cdot \infty=\infty \cdot 0=0$ is taken. In particular, note that $e^{-0 . z}=1$ for all $z \in[0, \infty]$. We first establish that if $\infty$ is inaccessible then it is an entrance boundary. 
Theorem 3.3 (Infinity as entrance boundary). Assume $\mathcal{E}=\infty$. The Markov process $\left(Z_{t}^{\min }, t \geq 0\right)$ can be extended in $[0, \infty]$ to a Feller process $\left(Z_{t}, t \geq 0\right)$ with $\infty$ as an entrance boundary. The boundary 0 is an exit of the diffusion $\left(U_{t}, t \geq 0\right)$ solution to (2.7), and the semi-group of $\left(Z_{t}, t \geq 0\right)$ satisfies for all $t \geq 0$, all $z \in[0, \infty]$, all $x \in[0, \infty)$

$$
\mathbb{E}_{z}\left(e^{-x Z_{t}}\right)=\mathbb{E}_{x}\left(e^{-z U_{t}}\right) .
$$

The proof of Theorem 3.3 is in Section 6 and relies on a duality at the level of generators between the minimal logistic CSBP with branching mechanism $\Psi$ and the $\Psi$-generalized Feller diffusion, see the forthcoming Lemma 5.1.

In the sequel, we focus on the case $\mathcal{E}<\infty$, for which $\infty$ is accessible. We will construct extensions of the minimal process as limits of non-explosive logistic CSBPs. For any Lévy measure $\pi$ and any $x \geq 0$, set $\bar{\pi}(x):=\pi([x, \infty))$. Given $\Psi$ of the form (2.3) and $k \geq 1$, set $\pi_{k}:=\pi_{\mid(0, k)}+(\bar{\pi}(k)+\lambda) \delta_{k}$ and define a branching mechanism $\Psi_{k}$ by

$$
\Psi_{k}(z):=\frac{\sigma^{2}}{2} z^{2}+\gamma z+\int_{0}^{\infty}\left(e^{-z x}-1+z x \mathbb{1}_{x \in(0,1)}\right) \pi_{k}(\mathrm{~d} x) .
$$

Plainly, for any $k \geq 1,\left|\Psi_{k}^{\prime}(0+)\right|<\infty$ and thus $\mathcal{E}_{k}:=\int_{0}^{\theta} \frac{1}{x} \exp \left(\frac{2}{c} \int_{x}^{\theta} \frac{\Psi_{k}(u)}{u} \mathrm{~d} u\right) \mathrm{d} x=\infty$. By Theorem 3.1, the minimal logistic CSBP with mechanism $\Psi_{k}$ does not explode. Theorem 3.3 ensures the existence of a càdlàg logistic CSBP, $\left(Z_{t}^{(k)}, t \geq 0\right)$, with mechanism $\Psi_{k}$ and $\infty$ as entrance boundary.

We will show that the sequence of processes $\left(Z_{t}^{(k)}, t \geq 0\right)$ converges towards a process $\left(Z_{t}, t \geq 0\right)$ with $\infty$ either regular or exit. The boundary will be regular, instantaneous and reflecting when $0 \leq \frac{2 \lambda}{c}<1$, and an exit when $\frac{2 \lambda}{c} \geq 1$.

Theorem 3.4 (Infinity as regular reflecting boundary). Assume $\mathcal{E}<\infty$ and $0 \leq \frac{2 \lambda}{c}<1$. The sequence of processes $\left(Z_{t}^{(k)}, t \geq 0\right)$ converges weakly in $\mathcal{D}([0, \infty])$ towards a Feller process $\left(Z_{t}, t \geq 0\right)$, extending $\left(Z_{t}^{\min }, t \geq 0\right)$, with $\infty$ regular instantaneous reflecting. The semi-group of $\left(Z_{t}, t \geq 0\right)$ satisfies for all $t \geq 0$, all $z \in[0, \infty]$ and $x \in[0, \infty)$,

$$
\mathbb{E}_{z}\left(e^{-x Z_{t}}\right)=\mathbb{E}_{x}\left(e^{-z U_{t}^{0}}\right)
$$

where $\left(U_{t}^{0}, t \geq 0\right)$ is solution to (2.7) with 0 regular absorbing.

Theorem 3.5 (Infinity as exit boundary). Assume $\frac{2 \lambda}{c} \geq 1$ (so that $\mathcal{E}<\infty$ ). The sequence of processes $\left(Z_{t}^{(k)}, t \geq 0\right)$ converges weakly in $\mathcal{D}([0, \infty])$ towards a Feller process $\left(Z_{t}, t \geq\right.$ $0)$, extending $\left(Z_{t}^{\min }, t \geq 0\right)$, with $\infty$ an exit. The boundary 0 is an entrance of the diffusion $\left(U_{t}, t \geq 0\right)$ solution to (2.7) and the semi-group of $\left(Z_{t}, t \geq 0\right)$ satisfies for all $t \geq 0$, all $z \in[0, \infty]$ and $x \in(0, \infty)$,

$$
\mathbb{E}_{z}\left(e^{-x Z_{t}}\right)=\mathbb{E}_{x}\left(e^{-z U_{t}}\right) .
$$

The proof of Theorem 3.4 and Theorem 3.5 can be found in Section 7. It relies on uniform convergence of semi-groups and generators for the sequence of processes $\left(Z_{t}^{(k)}, t \geq 0\right)$.

In the next statements, $\left(Z_{t}, t \geq 0\right)$ refers to the extended logistic CSBP valued in $[0, \infty]$, as defined above, whose boundary $\infty$ is either an entrance, regular reflecting, or an exit. The following corollary classifies the boundary 0 .

Corollary 3.6 (Zero as exit or natural boundary).

i) Assume $\int^{\infty} \frac{\mathrm{d} z}{|\Psi(z)|}<\infty$ then 0 is an exit boundary of $\left(Z_{t}, t \geq 0\right)$ and $\infty$ is an entrance boundary of $\left(U_{t}, t \geq 0\right)$.

ii) Assume $\int^{\infty} \frac{\mathrm{d} z}{|\Psi(z)|}=\infty$ then 0 is a natural boundary of $\left(Z_{t}, t \geq 0\right)$ and $\infty$ is a natural boundary of $\left(U_{t}, t \geq 0\right)$. 

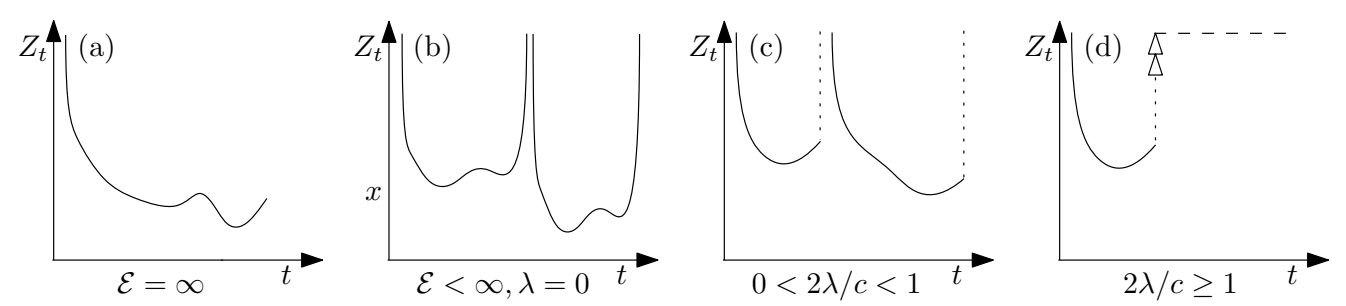

Figure 1: Symbolic representation of the four behaviors at $\infty$.

Table 1: Boundaries of $Z$ and boundaries of $U$.

\begin{tabular}{c|l} 
Boundary of $Z$ & Boundary of $U$ \\
\hline$\infty$ entrance & 0 exit \\
\hline$\infty$ regular reflecting & 0 regular absorbing \\
\hline$\infty$ exit & 0 entrance \\
\hline 0 exit & $\infty$ entrance \\
\hline 0 natural & $\infty$ natural \\
\hline
\end{tabular}

The boundary behaviors found in Theorems 3.3, 3.4 and 3.5 and Corollary 3.6 are summarized in Table 1.

When $-\Psi$ is the Laplace exponent of a subordinator (hence the associated CSBP has non-decreasing sample paths) and the boundary $\infty$ is regular or entrance, the logistic CSBP may have a stationary distribution. The next theorem yields a necessary and sufficient condition for a stationary distribution to exist and provides its Laplace transform.

Theorem 3.7 (Stationarity). Assume $\Psi(z)<0$ for all $z>0$, and write $\Psi$ in the form (2.4)

$$
\Psi(z)=-\lambda-\delta z-\int_{0}^{\infty}\left(1-e^{-z u}\right) \pi(\mathrm{d} u)
$$

with $\lambda \geq 0, \delta \geq 0$ and $\int_{0}^{\infty}(1 \wedge u) \pi(\mathrm{d} u)<\infty$. Assume $0 \leq \frac{2 \lambda}{c}<1$ and define the condition (A) as follows

$$
\text { (A) : }(\delta=0 \text { and } \bar{\pi}(0)+\lambda \leq c / 2) \text {. }
$$

- If (A) is satisfied then $\left(Z_{t}, t \geq 0\right)$ converges in probability to 0 .

- If (A) is not satisfied then $\left(Z_{t}, t \geq 0\right)$ converges in law towards the distribution supported on $\left(\frac{2 \delta}{c}, \infty\right)$ whose Laplace transform is

$$
L: x \in \mathbb{R}_{+} \mapsto \mathbb{E}\left[e^{-x Z_{\infty}}\right]:=\frac{\int_{x}^{\infty} \exp \left(\int_{\theta}^{y} \frac{2 \Psi(z)}{c z} \mathrm{~d} z\right) \mathrm{d} y}{\int_{0}^{\infty} \exp \left(\int_{\theta}^{y} \frac{2 \Psi(z)}{c z} \mathrm{~d} z\right) \mathrm{d} y} .
$$

Remark 3.8. Note that the formula (3.1) does not depend on $\theta$. Moreover, the condition in Theorem 3.7 for the existence of a non-degenerate stationary distribution can be rephrased as follows. The condition (A) is not satisfied if and only if at least one of the following holds

$$
\lim _{u \rightarrow \infty} \frac{\Psi(u)}{u}=-\delta \neq 0, \pi((0,1))=\infty, \bar{\pi}(0)+\lambda>\frac{c}{2} .
$$


This already appears with $\lambda=0$ and a moment assumption in [Lam05, Theorem 3.4]. One can easily see from the Laplace transform that $\lambda=0$ and $\int^{\infty} \log (x) \pi(\mathrm{d} x)<\infty$ are necessary and sufficient conditions for the stationary distribution to admit a first moment.

The next theorem completes Theorem 3.7 by classifying the long-term behavior of the logistic CSBP when $-\Psi$ is not the Laplace exponent of a subordinator or when the boundary $\infty$ is an exit. When $\infty$ is regular reflecting or an entrance and $-\Psi$ is not the Laplace exponent of a subordinator, the extended process $\left(Z_{t}, t \geq 0\right)$ gets extinct almost surely. When $\infty$ is an exit and $-\Psi$ is the Laplace exponent of a subordinator, the process gets absorbed at $\infty$ almost surely. Lastly, if $-\Psi$ is not the Laplace exponent of a subordinator then the process, with $\infty$ exit, has a positive probability of extinction.

Theorem 3.9 (Long-term behaviors). Consider $\left(Z_{t}, t \geq 0\right)$ started from $z \in(0, \infty)$.

1) If $0 \leq \frac{2 \lambda}{c}<1$ and $\Psi(z) \geq 0$ for some $z>0$ then

1-1) If $\int^{\infty} \frac{\mathrm{d} u}{\Psi(u)}=\infty$, then $Z_{t}>0$ for any $t \geq 0$ a.s. and $Z_{t} \underset{t \rightarrow \infty}{\longrightarrow} 0$ a.s.

1-2) If $\int^{\infty} \frac{\mathrm{d} u}{\Psi(u)}<\infty$, then $\left(Z_{t}, t \geq 0\right)$ will be absorbed at 0 in finite time almost surely.

2) If $\frac{2 \lambda}{c} \geq 1$ and $\Psi(z)<0$ for all $z>0$ then $\left(Z_{t}, t \geq 0\right)$ will be absorbed at $\infty$ in finite time almost surely.

3) If $\frac{2 \lambda}{c} \geq 1$ and $\Psi(z) \geq 0$ for some $z>0$ then

$$
\mathbb{P}_{z}\left(Z_{t} \underset{t \rightarrow \infty}{\longrightarrow} 0\right)=\frac{\int_{0}^{\infty} e^{-z u} \frac{1}{u} \exp \left(-\int_{\theta}^{u} \frac{2 \Psi(v)}{c v} \mathrm{~d} v\right) \mathrm{d} u}{\int_{0}^{\infty} \frac{1}{u} \exp \left(-\int_{\theta}^{u} \frac{2 \Psi(v)}{c v} \mathrm{~d} v\right) \mathrm{d} u} \in(0,1)
$$

and for any $t \geq 0, Z_{t}>0 \mathbb{P}_{z}$-almost surely if and only if $\int^{\infty} \frac{\mathrm{d} u}{\Psi(u)}=\infty$.
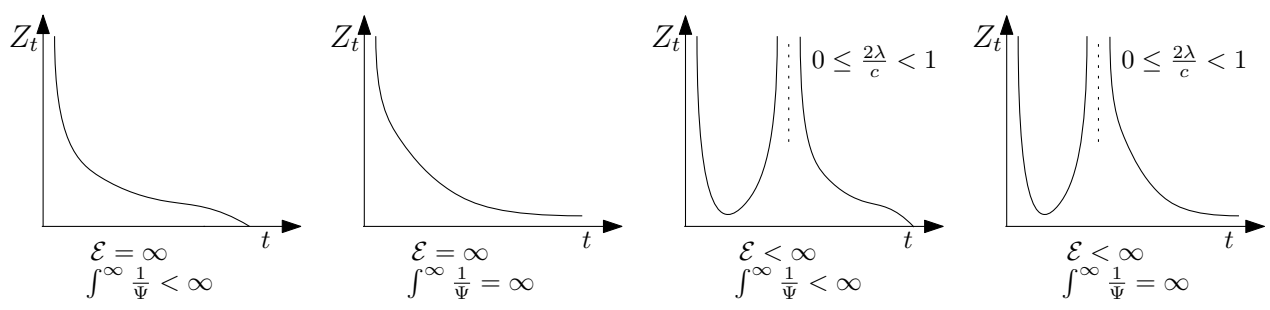

Figure 2: Symbolic representation of the two possible behaviors at 0 in the nonsubordinator case with $\infty$ entrance or reflecting.

The proofs of Corollary 3.6, Theorems 3.7 and 3.9 are handled separately in Section 6.2 and Section 7 according to $\mathcal{E}=\infty$ or $\mathcal{E}<\infty$, respectively.

We provide now several examples for which different behaviors at infinity occur.

Example 3.10. Consider $\alpha \in(0,2], \alpha \neq 1$ and $\Psi(z)=(\alpha-1) z^{\alpha}$. Since $\int_{0} \frac{|\Psi(z)|}{z} \mathrm{~d} z<\infty$, we have that $\mathcal{E}=\infty$ and $\infty$ is an entrance boundary (case (a) in Figure 1). For any $t \geq 0$, $z \in[0, \infty]$ and $x \in[0, \infty)$

$$
\mathbb{E}_{z}\left(e^{-x Z_{t}}\right)=\mathbb{E}_{x}\left(e^{-z U_{t}}\right) \text { with } \mathrm{d} U_{t}=\sqrt{c U_{t}} \mathrm{~d} B_{t}+(1-\alpha) U_{t}^{\alpha} \mathrm{d} t, U_{0}=x,
$$

the boundary 0 of $\left(U_{t}, t \geq 0\right)$ being an exit. As recalled in Section 2, when $\alpha \in(0,1)$, the CSBP without competition explodes, so that here competition prevents explosion. 
Moreover, when $\alpha \in(0,1)$, condition (A) is not satisfied and a computation from (3.1) yields

$$
L: x \mapsto \frac{\Gamma\left(1 / \alpha, c_{\alpha} x^{\alpha}\right)}{\Gamma(1 / \alpha)}
$$

with $c_{\alpha}=\frac{2}{c} \frac{1-\alpha}{\alpha}$ and $\Gamma(1 / \alpha, x):=\int_{x}^{\infty} e^{-t} t^{1 / \alpha-1} \mathrm{~d} t$ for any $x \geq 0$, is the incomplete Gamma function.

Example 3.11. Let $\lambda>0$ and $\Psi(x)=-\lambda$ for all $x \geq 0$.

i) If $\frac{2 \lambda}{c}<1$ then $\infty$ is regular reflecting (case (c) in Figure 1). For any $t \geq 0, z \in[0, \infty]$ and $x \in[0, \infty)$

$$
\mathbb{E}_{z}\left(e^{-x Z_{t}}\right)=\mathbb{E}_{x}\left(e^{-z U_{t}^{0}}\right) \text { with } \mathrm{d} U_{t}^{0}=\sqrt{c U_{t}^{0}} \mathrm{~d} B_{t}+\lambda \mathrm{d} t, U_{0}^{0}=x,
$$

the boundary 0 of $\left(U_{t}^{0}, t \geq 0\right)$ being regular absorbing.

ii) If $\frac{2 \lambda}{c} \geq 1$ then $\infty$ is an exit (case (d) in Figure 1). For any $t \geq 0, z \in[0, \infty]$ and $x \in(0, \infty)$

$$
\mathbb{E}_{z}\left(e^{-x Z_{t}}\right)=\mathbb{E}_{x}\left(e^{-z U_{t}}\right) \text { with } \mathrm{d} U_{t}=\sqrt{c U_{t}} \mathrm{~d} B_{t}+\lambda \mathrm{d} t, U_{0}=x,
$$

the boundary 0 of $\left(U_{t}, t \geq 0\right)$ being an entrance.

Remark 3.12. Roughly speaking, the latter example can be seen as the continuous-state analogue of the number of fragments in a fast-fragmentation-coalescence process as defined in [KPRS17]. Note in particular that the same phase transition between $\infty$ reflecting and exit boundary occurs. However, contrary to the process in [KPRS17], the process $\left(Z_{t}, t \geq 0\right)$ has no stationary distribution over $(0, \infty)$ as condition $(\mathbf{A})$ is satisfied when $\frac{2 \lambda}{c}<1$.

The conditions $\int_{0} \frac{|\Psi(x)|}{x} \mathrm{~d} x<\infty$ and $\lambda>0$ are not necessary for having respectively $\mathcal{E}=\infty$ and $\mathcal{E}<\infty$. The following proposition, whose proof can be found at the beginning of the Appendix, allows us to construct explicit Lévy measures for which $\infty$ is regular or entrance.

Proposition 3.13. Assume $\lambda=0$, the integrals $\int_{0} \frac{|\Psi(u)|}{u} \mathrm{~d} u$ and $\int^{\infty} \log (u) \pi(\mathrm{d} u)$ have the same nature. In particular $\int^{\infty} \log (u) \pi(\mathrm{d} u)<\infty$ implies $\mathcal{E}=\infty$. Set

$$
\mathcal{E}^{\prime}:=\int_{0}^{\theta} \frac{1}{x} \exp \left(-\frac{2}{c} \int_{1}^{\infty} e^{-x v} \frac{\bar{\pi}(v)}{v} \mathrm{~d} v\right) \mathrm{d} x
$$

then $\mathcal{E}<\infty$ if and only if $\mathcal{E}^{\prime}<\infty$. Moreover there exists a universal ${ }^{2}$ constant $\kappa>0$ and $C_{1}, C_{2}$ two non-negative constants such that

$$
C_{1} \int_{0}^{\theta} \frac{1}{x} \exp \left(-\frac{2 \kappa}{c} \int_{1}^{1 / x} \frac{\bar{\pi}(u)}{u} \mathrm{~d} u\right) \mathrm{d} x \leq \mathcal{E} \leq C_{2} \int_{0}^{\theta} \frac{1}{x} \exp \left(-\frac{2}{c \kappa} \int_{1}^{1 / x} \frac{\bar{\pi}(u)}{u} \mathrm{~d} u\right) \mathrm{d} x .
$$

Proposition 3.13 and a Tauberian theorem (see e.g Theorem 5.13 in [Kyp14]), yields the following example with $\lambda=0$. Note that a phase transition occurs between entrance and regular.

Example 3.14. Consider $\lambda=0, \sigma \geq 0, \gamma \in \mathbb{R}$ and set $\pi(\mathrm{d} u)=\frac{\alpha}{u(\log u)^{\beta+1}} \mathbb{1}_{\{u \geq e\}} \mathrm{d} u$ for some $\alpha>0, \beta>0$.

i) If $\beta>1$ or $\beta=1$ and $\frac{2 \alpha}{c} \leq 1$ then $\mathcal{E}=\infty$ and $\infty$ is an entrance boundary (case (a) in Figure 1).

\footnotetext{
${ }^{2}$ in the sense that it does not depend on the Lévy measure $\pi$
} 
ii) If $\beta=1$ and $\frac{2 \alpha}{c}>1$ then $\mathcal{E}<\infty$ and $\infty$ is a regular reflecting boundary (case (b) in Figure 1).

iii) If $\beta \in(0,1)$, then $\mathcal{E}<\infty$ and $\infty$ is a regular reflecting boundary (case (b) in Figure 1).

If $\Psi$ is in the form (7) with $\delta=0$ then the process has a stationary distribution if and only if $\bar{\pi}(0)=\frac{\alpha}{\beta}>\frac{c}{2}$.

\section{Minimal process and time-change}

We will prove in this section that the martingale problem (MP) for the minimal logistic CSBP is well-posed. We study then the event of extinction and explosion of the minimal process. In particular Theorem 3.1 follows from the forthcoming Lemma 4.5. As described in [Lam05, Definition 3.2], one way to construct a logistic CSBP is to start from an Ornstein-Uhlenbeck type process and to time change it in Lamperti's manner. The problem of explosion was not addressed in [Lam05] and lies at the heart of our study. We provide therefore some details. We start by recalling some known results about Ornstein-Uhlenbeck type processes. Consider $\left(Y_{t}, t \geq 0\right)$ a spectrally positive Lévy process with Laplace exponent $-\Psi$, killed at $\infty$ at an independent exponential random variable $\mathbf{e}_{\lambda}$ with parameter $\lambda:=-\Psi(0) \geq 0$. Set $\left(R_{t}, t \geq 0\right)$ the process satisfying

$$
R_{t}=z+Y_{t}-\frac{c}{2} \int_{0}^{t} R_{s} \mathrm{~d} s .
$$

There is a unique process $\left(R_{t}, t \geq 0\right)$ satisfying (4.1), see Sato [Sat13, Chapter 3, Section 17 page 104]. By definition it is called an Ornstein-Uhlenbeck type process with Lévy process $\left(Y_{t}, t \geq 0\right)$ and parameter $c / 2$. The process $\left(R_{t}, t \geq 0\right)$ can be written as follows

$$
R_{t}=z e^{-\frac{c}{2} t}+\int_{0}^{t} e^{-\frac{c}{2}(t-s)} \mathrm{d} Y_{s} .
$$

Unkilled Ornstein-Uhlenbeck type processes have been deeply studied by Hadjiev [Had85], Sato and Yamazato [SY84] and Shiga [Shi90]. From Lemma 17.1 in Sato [Sat13], one has for any $\theta>0$ and any $s \geq 0$

$$
\mathbb{E}_{z}\left(e^{-\theta R_{s}}\right)=\exp \left(-\theta e^{-\frac{c}{2} s} z+\int_{0}^{s} \Psi\left(e^{-\frac{c}{2} u} \theta\right) \mathrm{d} u\right) .
$$

In particular, by letting $\theta$ to 0 , we see that the process $\left(R_{t}, t \geq 0\right)$ will never reach $\infty$ in finite time if it is not killed. In the unkilled case, it is shown in [Shi90] that if $\left(Y_{t}, t \geq 0\right)$ is not a subordinator then the process $\left(R_{t}, t \geq 0\right)$ is irreducible in $\mathbb{R}$. Namely, for any $a \in \mathbb{R}$, if $\sigma_{a}:=\inf \left\{t \geq 0, R_{t} \leq a\right\}$ then $\mathbb{P}_{z}\left(\sigma_{a}<\infty\right)>0$ for any $z>0$. On the other hand, if $-\Psi$ is the Laplace exponent of a subordinator with drift $\delta \geq 0$, then the process $\left(R_{t}, t \geq 0\right)$ is irreducible in $\left(\frac{2 \delta}{c}, \infty\right)$. Moreover, the process can be positive recurrent, null-recurrent or transient. [Shi90, Theorem 1.1] states that $\left(R_{t}, t \geq 0\right)$ is recurrent (in a pointwise sense) if $\mathcal{E}=\infty$ and transient if $\mathcal{E}<\infty$, where we recall

$$
\mathcal{E}=\int_{0}^{\theta} \frac{1}{x} \exp \left(\frac{2}{c} \int_{x}^{\theta} \frac{\Psi(u)}{u} \mathrm{~d} u\right) \mathrm{d} x .
$$

If $\lambda=-\Psi(0)>0$, then one can easily see that $\mathcal{E}<\infty$, so that explosion by a single jump can be seen as a particular case of transience.

We work in the sequel with the process $\left(R_{t}, t \geq 0\right)$ stopped on first entry into $(-\infty, 0)$. For any $a \in[0, \infty)$, define $\sigma_{a}:=\inf \left\{t \geq 0, R_{t} \leq a\right\}$. Since $R$ has no negative jumps, 
$\sigma_{a}=\inf \left\{t \geq 0, R_{t}<a\right\}$ a.s. Define the additive functional,

$$
t \mapsto \theta_{t}:=\int_{0}^{t \wedge \sigma_{0}} \frac{\mathrm{d} s}{R_{s}} \in[0, \infty] .
$$

We first check that for any $t<\sigma_{0}, \theta_{t}<\infty$ a.s. Note that $\left(\sigma_{1 / n}, n \geq 1\right)$ increases towards $\sigma_{0}$ almost surely as $n$ goes to $\infty$ and for any $t<\sigma_{0}$, there exists a large enough $n$ such that $t<\sigma_{1 / n}$ almost surely. Therefore, $\inf _{s \leq t} R_{s} \geq \inf _{s \leq \sigma_{1 / n}} R_{s} \geq 1 / n$ a.s. and $\theta_{t} \leq n t<\infty$ a.s. Define now the right-inverse of $\left(\theta_{t}, t \geq 0\right)$

$$
t \mapsto C_{t}:=\inf \left\{u \geq 0: \theta_{u}>t\right\} \in[0, \infty] .
$$

The Lamperti time-change of the stopped process $\left(R_{t}, t \geq 0\right)$ is the process $\left(Z_{t}^{\min }, t \geq 0\right)$ defined by

$$
Z_{t}^{\min }= \begin{cases}R_{C_{t}} & 0 \leq t<\theta_{\infty} \\ 0 & t \geq \theta_{\infty} \text { and } \sigma_{0}<\infty \\ \infty & t \geq \theta_{\infty} \text { and } \sigma_{0}=\infty\end{cases}
$$

A first consequence of this definition is that the process $\left(Z_{t}^{\min }, t \geq 0\right)$ hits its boundaries if and only if $\theta_{\infty}<\infty$. Recall $\zeta_{\infty}$ and set $\zeta_{0}:=\inf \left\{t \geq 0, Z_{t}^{\min }=0\right\}$. On the one hand, if $\sigma_{0}<\infty$ then $\zeta_{\infty}=\infty$ and $\zeta_{0}=\theta_{\infty}=\int_{0}^{\sigma_{0}} \frac{\mathrm{d} s}{R_{s}}$. On the other hand, if $\sigma_{0}=\infty$ then $\zeta_{0}=\infty$ and $\zeta_{\infty}=\theta_{\infty}=\int_{0}^{\infty} \frac{\mathrm{d} s}{R_{s}}$. Note that if $\lambda>0$, then $R_{s}=\infty$ for any $s \geq \mathbf{e}_{\lambda}$ and the last integral is finite.

Recall (2.6) and the martingale problem (MP) defining the minimal logistic CSBP.

Lemma 4.1. The process $\left(Z_{t}^{\min }, t \geq 0\right)$ is a minimal logistic continuous-state branching process.

Proof. Notice first that if the process $\left(Z_{t}^{\min }, t \geq 0\right)$, as defined above, hits 0 or $\infty$, then it is absorbed. Denote by $\mathscr{L}^{Y}$ the generator of the (possibly killed) Lévy process $\left(Y_{t}, t \geq 0\right)$ and $\mathscr{L}^{R}$ the generator of $\left(R_{t}, t \geq 0\right)$, which acts on $C_{c}^{2}([0, \infty))$ as follows

$$
\mathscr{L}^{R} f(z)=\mathscr{L}^{Y} f(z)-\frac{c}{2} z f^{\prime}(z) .
$$

By Itô's formula (or by applying [SY84, Theorem 3.1]), one can see that the process $\left(f\left(R_{t}\right)-\int_{0}^{t} \mathscr{L}^{R} f\left(R_{s}\right) \mathrm{d} s, t \geq 0\right)$ is a local martingale. By definition of the time-change, for any $t \in\left[0, \theta_{\infty}\right), \int_{0}^{C_{t}} \frac{\mathrm{d} s}{R_{s}}=t$ and then $C_{t}=\int_{0}^{t} Z_{s}^{\min } \mathrm{d} s$. A (continuous) time-change of a local martingale remains a local martingale, see e.g [RY99, Proposition V.1.5]. Hence

$$
t \in\left[0, \theta_{\infty}\right) \mapsto f\left(R_{C_{t}}\right)-\int_{0}^{C_{t}} \mathscr{L}^{R} f\left(R_{s}\right) \mathrm{d} s=f\left(Z_{t}^{\min }\right)-\int_{0}^{t} Z_{s}^{\min } \mathscr{L}^{R} f\left(Z_{s}^{\min }\right) \mathrm{d} s
$$

is a local martingale. By definition, for any $z \geq 0, \mathscr{L} f(z)=z \mathscr{L}^{R} f(z)$ and since $f$ has compact support, $\mathscr{L} f$ is bounded. Therefore the above local martingale has paths which are bounded on time-intervals $[0, t]$, so that it is a true martingale and $\left(Z_{t}^{\min }, t \geq 0\right)$ solves (MP).

\section{Lemma 4.2. There exists a unique minimal logistic CSBP.}

Proof. We have seen above how to construct a solution to the martingale problem. Only uniqueness has to be justified. Consider any solution $\left(Z_{t}, t<\zeta\right)$ to the martingale problem (MP). Set $C_{t}:=\int_{0}^{t} Z_{s} \mathrm{~d} s$ for $t<\zeta$ and $C_{t}:=C_{\zeta}$ for all $t \geq \zeta$. Let $\theta_{t}:=\inf \{s \geq$ $\left.0: C_{s}>t\right\}$ and $R_{t}:=Z_{\theta_{t}}$ for any time $t \in\left[0, C_{\zeta}\right)$. By definition, $R_{C_{t}}=Z_{t}$ and thus 
$C_{\zeta}=\inf \left\{t \geq 0 ; R_{t} \notin(0, \infty)\right\}$. As in Lemma 4.1, but in the opposite direction, one sees that the process $\left(R_{t}, t<C_{\zeta}\right)$ solves the same martingale problem as an OrnsteinUhlenbeck type process (with parameters $\tilde{\Psi}=\Psi+\lambda$ and $c / 2$ ) stopped on first entry into $(-\infty, 0)$. The Ornstein-Uhlenbeck type process is uniquely defined in law (see [Sat13, Chapter 3, section 17] where existence and pathwise uniqueness of the solution to (4.1) are established). Moreover, one can readily check that $C_{t}=\inf \left\{s \geq 0, \int_{0}^{s} \frac{\mathrm{d} u}{R_{u}}>t\right\}$, which entails that the law of $\left(Z_{t}, t \geq 0\right)$ is uniquely determined by the law of $\left(R_{t}, t \geq 0\right)$.

We now gather some path properties of minimal logistic CSBPs obtained directly by time-change.

Lemma 4.3. Assume that $-\Psi$ is not the Laplace exponent of a subordinator. If $\mathcal{E}=\infty$, then for any $z>0$

$$
\mathbb{P}_{z}\left(Z_{t}^{\min } \underset{t \rightarrow \infty}{\longrightarrow} 0\right)=1
$$

If $\mathcal{E}<\infty$, then

$$
\mathbb{P}_{z}\left(Z_{t}^{\min } \underset{t \rightarrow \infty}{\longrightarrow} 0\right)=\frac{\int_{0}^{\infty} \frac{1}{x} e^{-z x-\int_{\theta}^{x} \frac{2 \Psi(y)}{c y} \mathrm{~d} y} \mathrm{~d} x}{\int_{0}^{\infty} \frac{1}{x} e^{-\int_{\theta}^{x} \frac{2 \Psi(y)}{c y} \mathrm{~d} y} \mathrm{~d} x} \in(0,1) .
$$

Proof. By construction, $\left\{Z_{t}^{\text {min }} \underset{t \rightarrow \infty}{\longrightarrow} 0\right\}=\left\{\sigma_{0}<\infty\right\}$ with $\sigma_{0}:=\inf \left\{t \geq 0, R_{t} \leq 0\right\}$. According to Patie [Pat05, Proposition 3], for any $z>a \geq 0$ and $\mu>0$

$$
\mathbb{E}_{z}\left[e^{-\mu \sigma_{a}}\right]=\frac{\int_{0}^{\infty} x^{\frac{2 \mu}{c}-1} e^{-z x-\int_{\theta}^{x} \frac{2 \Psi(y)}{c y} \mathrm{~d} y} \mathrm{~d} x}{\int_{0}^{\infty} x^{\frac{2 \mu}{c}-1} e^{-a x-\int_{\theta}^{x} \frac{2 \Psi(y)}{c y} \mathrm{~d} y} \mathrm{~d} x} .
$$

For the sake of completeness, a short proof of (4.5) is provided at the end of the Appendix. We show now that if $\mathcal{E}=\infty$, then for any $z \in(0, \infty), \mathbb{P}_{z}\left(\sigma_{0}<\infty\right)=1$. For any $\mu \geq 0$, set

$$
g_{\mu}(x):=x^{\frac{2 \mu}{c}-1} e^{-\int_{\theta}^{x} \frac{2 \Psi(y)}{c y} \mathrm{~d} y} .
$$

We first check that for any $\mu \geq 0$ and any $a>0, \int_{a}^{\infty} g_{\mu}(x) \mathrm{d} x<\infty$. By assumption $-\Psi$ is not the Laplace exponent of a subordinator, so there exists $b \in(a, \infty)$ such that for all $u \geq b, \Psi(u) \geq \Psi(b)>\mu$. Then, for some constant $C$,

$$
\begin{aligned}
\int_{b}^{\infty} g_{\mu}(x) \mathrm{d} x & =C \int_{b}^{\infty} x^{\frac{2 \mu}{c}-1} e^{-\int_{b}^{x} \frac{2 \Psi(u)}{c u} \mathrm{~d} u} \mathrm{~d} x \leq C \int_{b}^{\infty} x^{\frac{2 \mu}{c}-1} e^{-\int_{b}^{x} \frac{2 \Psi(b)}{c u} \mathrm{~d} u} \mathrm{~d} x \\
& \leq C \int_{b}^{\infty} x^{-\left(\frac{2 \Psi(b)}{c}-\frac{2 \mu}{c}\right)-1} \mathrm{~d} x<\infty
\end{aligned}
$$

and $\int_{a}^{\infty} g_{\mu}(x) \mathrm{d} x=\int_{a}^{b} g_{\mu}(x) \mathrm{d} x+\int_{b}^{\infty} g_{\mu}(x) \mathrm{d} x<\infty$. By (4.5),

$$
\mathbb{E}_{z}\left[e^{-\mu \sigma_{0}}\right]=\frac{\int_{0}^{\infty} e^{-z x} g_{\mu}(x) \mathrm{d} x}{\int_{0}^{\infty} g_{\mu}(x) \mathrm{d} x} \geq \frac{\int_{0}^{a} e^{-z x} g_{\mu}(x) \mathrm{d} x}{\int_{0}^{a} g_{\mu}(x) \mathrm{d} x+\int_{a}^{\infty} g_{\mu}(x) \mathrm{d} x} \geq e^{-a z} \frac{1}{1+\frac{\int_{a}^{\infty} g_{\mu}(x) \mathrm{d} x}{\int_{0}^{a} g_{\mu}(x) \mathrm{d} x}} .
$$

For any $\mu \leq \frac{c}{4}$, and $x \geq a, g_{\mu}(x)=x^{\frac{2 \mu}{c}-1} g_{0}(x) \leq a^{-1 / 2} g_{0}(x)$. By Lebesgue's theorem $\lim _{\mu \rightarrow 0} \int_{a}^{\infty} g_{\mu}(\bar{x}) \mathrm{d} x=\int_{a}^{\infty} g_{0}(x) \mathrm{d} x<\infty$. For any $a \in(0,1]$ and any $x \in(0, a), g_{\mu}(x)$ increases towards $g_{0}(x)$ as $\mu$ goes to 0 . An application of the monotone convergence theorem yields that $\lim _{\mu \rightarrow 0} \int_{0}^{a} g_{\mu}(x) \mathrm{d} x=\int_{0}^{a} g_{0}(x) \mathrm{d} x$, which is infinite since by assumption $\mathcal{E}=\infty$. Therefore,

$$
\frac{\int_{a}^{\infty} g_{\mu}(x) \mathrm{d} x}{\int_{0}^{a} g_{\mu}(x) \mathrm{d} x} \underset{\mu \rightarrow 0}{\longrightarrow} 0
$$


and the inequality (4.6) entails $\mathbb{P}_{z}\left(\sigma_{0}<\infty\right) \geq e^{-a z}$ for any $a \in(0,1]$. One concludes that $\mathbb{P}_{z}\left(\sigma_{0}<\infty\right)=1$. Similar arguments show that if $\mathcal{E}<\infty$, then

$$
\mathbb{P}_{z}\left(\sigma_{0}<\infty\right)=\lim _{\mu \rightarrow 0} \mathbb{E}_{z}\left[e^{-\mu \sigma_{0}}\right]=\frac{\int_{0}^{\infty} x^{-1} e^{-z x-\int_{\theta}^{x} \frac{2 \Psi(y)}{c y} \mathrm{~d} y} \mathrm{~d} x}{\int_{0}^{\infty} x^{-1} e^{-\int_{\theta}^{x} \frac{2 \Psi(y)}{c y} \mathrm{~d} y} \mathrm{~d} x} \in(0,1) .
$$

The time-change allows one to get directly some interesting identities. Here we give the Laplace transform of the so-called total progeny.

Lemma 4.4. Assume that $-\Psi$ is not the Laplace exponent of a subordinator. Set $\zeta_{a}:=$ $\inf \left\{t \geq 0 ; Z_{t}^{\min } \leq a\right\}$. For any $z>a \geq 0$ and $\mu>0$, one has

$$
\mathbb{E}_{z}\left[e^{-\mu \int_{0}^{\zeta a} Z_{s}^{\min } \mathrm{d} s}\right]=\frac{\int_{0}^{\infty} x^{\frac{2 \mu}{c}-1} e^{-z x-\int_{\theta}^{x} \frac{2 \Psi(y)}{c y} \mathrm{~d} y} \mathrm{~d} x}{\int_{0}^{\infty} x^{\frac{2 \mu}{c}-1} e^{-a x-\int_{\theta}^{x} \frac{2 \Psi(y)}{c y} \mathrm{~d} y} \mathrm{~d} x} .
$$

Proof. By the time-change transformation from $\left(R_{t}, t \geq 0\right)$ to $\left(Z_{t}^{\min }, t \geq 0\right)$, we have that $\sigma_{a}=\int_{0}^{\zeta_{a}} Z_{s}^{\min } \mathrm{d} s$ a.s and the statement follows directly by (4.5).

The next lemma establishes Theorem 3.1.

Lemma 4.5 (Explosion). The minimal process explodes with positive probability if and only if $\mathcal{E}<\infty$.

Proof. On the event $\left\{\sigma_{0}<\infty\right\},\left(Z_{t}^{\min }, t \geq 0\right)$ converges towards 0 almost surely and thus does not explode. We then focus on the event $\left\{\sigma_{0}=\infty\right\}$. If $\lambda>0$ then explosion is trivial. Assume now $\lambda=0$. By construction, the process $\left(Z_{t}^{\min }, t \geq 0\right)$ explodes if and only if $\int^{\infty} \frac{1}{R_{s}} \mathrm{~d} s<\infty$.

Assume first $\mathcal{E}=\infty$. Recall that [Shi90, Theorem 1.1] ensures that the process $\left(R_{t}, t \geq 0\right)$ is recurrent. Let $a>0$ and $b_{0}:=0$, consider the stopping times $a_{n}:=\inf \{t>$ $\left.b_{n-1}, R_{t} \leq a\right\}$ and $b_{n}:=\inf \left\{t>a_{n}, R_{t}>2 a\right\}$ for any $n \geq 1$. By recurrence, for any $n \geq 1$, $a_{n}<\infty$ and $b_{n}<\infty$ almost surely. We see that

$$
\int_{0}^{\infty} \frac{\mathrm{d} s}{R_{s}} \geq \sum_{n \geq 1} \int_{a_{n}}^{b_{n}} \frac{1}{R_{s}} \mathrm{~d} s \geq \sum_{n \geq 1} \frac{b_{n}-a_{n}}{2 a} .
$$

By the Markov property, the random variables $\left(b_{n}-a_{n}, n \geq 1\right)$ are i.i.d and positive. Plainly

$$
\mathbb{E}_{z}\left[e^{-\int_{0}^{\infty} \frac{\mathrm{d} s}{R_{s}}}, \sigma_{0}=\infty\right] \leq \mathbb{E}_{z}\left[e^{-\frac{1}{a} \sum_{n \geq 1}\left(b_{n}-a_{n}\right)}\right]=0 .
$$

Therefore $\theta_{\infty}=\infty$. Hence, $Z_{t}^{\min }<\infty$ for all $t>0$ and $\infty$ is inaccessible.

Consider now the case $\mathcal{E}<\infty$, the unstopped process $\left(R_{t}, t \geq 0\right)$ is transient, and the event $\left\{\sigma_{0}=\infty\right\}$ has positive probability. We check that the integral $\int_{0}^{\infty} \frac{1}{R_{s}} \mathrm{~d} s$ is finite almost surely on the event $\left\{\sigma_{0}=\infty\right\}$. Recall the Laplace transform (4.3), one has

$$
\mathbb{E}_{z}\left(e^{-\theta R_{s}}\right)=\exp \left(-\theta e^{-\frac{c}{2} s} z+\int_{0}^{s} \Psi\left(e^{-\frac{c}{2} u} \theta\right) \mathrm{d} u\right)
$$

By the change of variables $v=e^{-\frac{c}{2} u} \theta$, we get $\int_{0}^{s} \Psi\left(e^{-\frac{c}{2} u} \theta\right) \mathrm{d} u=\int_{\theta e^{-\frac{2}{c} s}}^{\theta} \frac{2 \Psi(v)}{c v} \mathrm{~d} v$, therefore

$$
\mathbb{E}_{z}\left(e^{-\theta R_{s}}\right)=\exp \left(-\theta e^{-\frac{c}{2} s} z+\int_{\theta e^{-\frac{c}{2} s}}^{\theta} \frac{2 \Psi(v)}{c v} \mathrm{~d} v\right)
$$

and the same change of variables $x=\theta e^{-\frac{c}{2} s}$ provides,

$$
\int_{0}^{\infty} \mathbb{E}_{z}\left(e^{-\theta R_{s}}\right) \mathrm{d} s=\frac{2}{c} \int_{0}^{\theta} \frac{1}{x} \exp \left(-x z+\int_{x}^{\theta} \frac{2 \Psi(v)}{c v} \mathrm{~d} v\right) \mathrm{d} x .
$$


Since $\mathcal{E}<\infty$, the last integral is finite for any $\theta>0$. Let $b>0$. By Tonelli, one has

$$
\begin{aligned}
\int_{0}^{\infty} \mathbb{E}_{z}\left(\frac{1-e^{-b R_{s}}}{R_{s}}, \sigma_{0}=\infty\right) \mathrm{d} s & =\int_{0}^{b} \int_{0}^{\infty} \mathbb{E}_{z}\left(e^{-\theta R_{s}}, \sigma_{0}=\infty\right) \mathrm{d} s \mathrm{~d} \theta \\
& \leq \int_{0}^{b} \int_{0}^{\infty} \mathbb{E}_{z}\left(e^{-\theta R_{s}}\right) \mathrm{d} s \mathrm{~d} \theta \\
& =\frac{2}{c} \int_{0}^{b} \mathrm{~d} \theta \int_{0}^{\theta} \frac{1}{x} \exp \left(-x z+\int_{x}^{\theta} \frac{2 \Psi(v)}{c v} \mathrm{~d} v\right) \mathrm{d} x
\end{aligned}
$$

The upper bound is finite since $\theta \in(0, b) \mapsto \int_{0}^{\theta} \frac{1}{x} e^{\int_{x}^{\theta} \frac{2 \Psi(v)}{c v} \mathrm{~d} v} \mathrm{~d} x$ is bounded. Thus

$$
\mathbb{E}_{z}\left(\int_{0}^{\infty} \frac{1-e^{-b R_{s}}}{R_{s}} \mathrm{~d} s, \sigma_{0}=\infty\right)<\infty
$$

We deduce then that on the event $\left\{\sigma_{0}=\infty\right\}, \int_{0}^{\infty} \frac{1-e^{-b R_{s}}}{R_{s}} \mathrm{~d} s<\infty$ a.s. Since $\mathcal{E}<\infty$, $R_{s} \underset{s \rightarrow \infty}{\longrightarrow} \infty$ a.s on the event $\left\{\sigma_{0}=\infty\right\}$ and $\frac{1-e^{-b R_{s}}}{R_{s}} \underset{s \rightarrow \infty}{\sim} \frac{1}{R_{s}}$ a.s. Therefore

$$
\mathbb{P}_{z}\left(\int_{0}^{\infty} \frac{\mathrm{d} s}{R_{s}}<\infty \mid \sigma_{0}=\infty\right)=1,
$$

and the process $\left(Z_{t}^{\min }, t \geq 0\right)$ explodes.

We have seen in Lemma 4.3 that extinction occurs with positive probability when $\mathcal{E}<\infty$ and $-\Psi$ is not the Laplace exponent of a subordinator. Therefore, there is a positive probability for the process to not explode. We see now that explosion is almost sure in the subordinator case.

Lemma 4.6. When $\mathcal{E}<\infty$, explosion is almost sure if and only if $-\Psi$ is the Laplace exponent of a subordinator.

Proof. We have seen in the proof of Lemma 4.5 that when $\mathcal{E}<\infty$, the following two events coincide

$$
\left\{\zeta_{\infty}=\infty\right\}=\left\{\sigma_{0}<\infty\right\} .
$$

In the non-subordinator case, one has $\mathbb{P}_{z}\left(\sigma_{0}=\infty\right)<1$ since the unstopped process $\left(R_{t}, t \geq 0\right)$ is irreducible in $(-\infty, \infty]$. Assume that $-\Psi$ is the Laplace exponent of a subordinator with drift $\delta \geq 0$ (possibly killed at rate $\lambda$ ). We show that $\sigma_{0}=\infty$ a.s. Let $\left(Y_{t}, t \geq 0\right)$ denote the subordinator with Laplace exponent $-\Psi$. Since $Y_{t} \geq z+\delta t$ for all $t \geq 0 \mathbb{P}_{z}$-a.s, a comparison argument in (4.1) entails that $R_{t} \geq r_{t}$ for all $t \geq 0$, $\mathbb{P}_{z}$-a.s, with $\left(r_{t}, t \geq 0\right)$ the solution to $\mathrm{d} r_{t}=\delta \mathrm{d} t-\frac{c}{2} r_{t} \mathrm{~d} t$ with $r_{0}=z$. We deduce that $R_{t} \geq e^{-\frac{c}{2} t} z+\frac{2 \delta}{c}\left(1-e^{-\frac{c}{2} t}\right)>0$ for all $t \geq 0, \mathbb{P}_{z}$-a.s. This entails $\mathbb{P}_{z}\left(\sigma_{0}=\infty\right)=1$ for any $z>0$.

Remark 4.7. When $-\Psi$ is the Laplace exponent of a subordinator, the Ornstein-Uhlenbeck type process is irreducible in $\left(\frac{2 \delta}{c}, \infty\right)$. Namely, for any $z \in\left(\frac{2 \delta}{c}, \infty\right)$, the process starting from $z$ hits any value in $\left(\frac{2 \delta}{c}, \infty\right)$ with positive probability. Since $\left(Z_{t}^{\min }, 0 \leq t<\zeta_{\infty}\right)$ is obtained by time-change of $\left(R_{t}, t \geq 0\right)$, it is therefore also irreducible in $\left(\frac{2 \delta}{c}, \infty\right)$.

When $\lambda>0$, the process has a positive probability to explode by a jump and one may wonder if it can explode continuously. We show in the next lemma that this is not possible. In other words, the two types of explosion (continuous and by a jump) cannot occur for a given process.

Lemma 4.8. If $\lambda>0$, then the minimal process always explodes by a jump to $\infty$. 
Proof. Let $\left(Z_{t}^{\min }, t \geq 0\right)$ be a minimal logistic CSBP with $\lambda>0$. By the time-change, $\left(R_{t}, t \geq 0\right):=\left(Z_{\theta_{t}}^{\min }, t \geq 0\right)$ is an Ornstein-Uhlenbeck type process killed at some exponential random variable $\mathbf{e}_{\lambda}$ and stopped at its first entry in $(-\infty, 0)$. Since for any $s<\mathbf{e}_{\lambda}$, $R_{s}<\infty$, we have that $Z_{t}^{\min }=R_{C_{t}}<\infty$ for any $t<\theta_{\mathbf{e}_{\lambda}}$. Therefore, the process cannot explode before $\theta_{\mathbf{e}_{\lambda}}$ and on the event $\left\{\sigma_{0}=\infty\right\}$, explosion is made by a single jump which occurs at time $\theta_{\mathbf{e}_{\lambda}}=\int_{0}^{\mathbf{e}_{\lambda}} \frac{\mathrm{d} s}{R_{s}}$.

Remark 4.9. We have seen that when the Ornstein-Uhlenbeck type process $\left(R_{t}, t \geq 0\right)$ is transient, the logistic CSBP explodes. Therefore, a logistic CSBP cannot grow indefinitely without exploding. This is a striking difference with CSBPs where indefinite growth with no explosion can occur when the Lévy process $\left(Y_{t}, t \geq 0\right)$ drifts "slowly" towards $\infty$.

\section{$5 \quad \Psi$-generalized Feller diffusions}

Consider a branching mechanism $\Psi$ as in (2.3). Recall that by $\Psi$-generalized Feller diffusion, we mean the diffusion $\left(U_{t}, t<\tau\right)$ with $\tau:=\inf \left\{t \geq 0 ; U_{t} \notin(0, \infty)\right\}$ solution to the sde

$$
U_{t}=x+\int_{0}^{t} \sqrt{c U_{s}} \mathrm{~d} B_{s}-\int_{0}^{t} \Psi\left(U_{s}\right) \mathrm{d} s
$$

where $\left(B_{t}, t \geq 0\right)$ is a Brownian motion. For all $x \in[0, \infty)$ and $z \in[0, \infty)$, set

$$
e_{x}(z):=e^{-x z} .
$$

The following observation is our starting point in the study of logistic continuous-state branching processes by duality.

Lemma 5.1 (Generator duality). For all $x \in[0, \infty)$ and $z \in[0, \infty)$,

$$
\mathscr{L} e_{x}(z)=\Psi(x) z e_{x}(z)+\frac{c}{2} x z^{2} e_{x}(z)=\mathscr{A} e_{z}(x)
$$

with

$$
\mathscr{A} f(x):=\frac{c}{2} x f^{\prime \prime}(x)-\Psi(x) f^{\prime}(x) .
$$

Proof. Recall $\mathscr{L}$ in (2.5). One can readily check that for all $x$ and $z$ in $[0, \infty)$,

$$
\mathscr{L} e_{x}(z)=\Psi(x) z e_{x}(z)+\frac{c}{2} x z^{2} e_{x}(z)=-\Psi(x) \frac{\partial e_{z}(x)}{\partial x}+\frac{c}{2} x \frac{\partial^{2} e_{z}(x)}{\partial x^{2}} .
$$

Intuitively, integrating each side in (5.2) should provide a duality at the level of semigroups of the form:

$$
\mathbb{E}_{z}\left(e^{-x Z_{t}^{\min }}\right)=\mathbb{E}_{x}\left(e^{-z U_{t}}\right) .
$$

The study of the boundaries 0 and $\infty$ of $\left(U_{t}, t \geq 0\right)$ would then provide the nature of boundaries $\infty$ and 0 of $\left(Z_{t}^{\min }, t \geq 0\right)$. However, there is not a unique semi-group associated with $\mathscr{A}$ as several boundary conditions are possible. Some precautions are then needed while showing the above duality.

We gather in the sequel, the boundary conditions of the diffusion. The proofs of the following statements are rather technical and postponed until the Appendix.

Lemma 5.2 (Boundaries). The boundaries 0 and $\infty$ of the diffusion with generator $\mathscr{A}$ are classified as follows:

1) The boundary 0 is an exit if $\mathcal{E}=\int_{0}^{\theta} \frac{1}{x} \exp \left(\frac{2}{c} \int_{x}^{\theta} \frac{\Psi(u)}{u} \mathrm{~d} u\right) \mathrm{d} x=\infty$, regular if $\mathcal{E}<\infty$ and $0 \leq \frac{2 \lambda}{c}<1$, and an entrance if $\frac{2 \lambda}{c} \geq 1$. 
2) The boundary $\infty$ is inaccessible. It is an entrance boundary if $\int^{\infty} \frac{\mathrm{d} x}{|\Psi(x)|}<\infty$ and a natural one if $\int^{\infty} \frac{\mathrm{d} x}{|\Psi(x)|}=\infty$.

When $\mathcal{E}<\infty$, the boundary 0 is regular and there are several possibilities for extending the minimal diffusion after $\tau$. In the next lemma, we denote by $\left(U_{t}^{0}, t \geq 0\right)$ the diffusion (5.1) with 0 either regular absorbing (if $\mathcal{E}<\infty$ ) or exit (if $\mathcal{E}=\infty$ ). This diffusion may have different long-term behaviors according to the branching mechanism $\Psi$. We classify these behaviors, stating in particular necessary and sufficient conditions over $\Psi$ for the process to escape to $\infty$ with positive probability.

Lemma 5.3 (Exit law from $(0, \infty)$ ). Assume $0 \leq \frac{2 \lambda}{c}<1$.

1) Assume there exists $z>0$, such that $\Psi(z) \geq 0$ (i.e $-\Psi$ is not the Laplace exponent of a subordinator), then for all $x \geq 0$,

$$
\mathbb{P}_{x}\left(\lim _{t \rightarrow \infty} U_{t}^{0}=0\right)=1 .
$$

2) Assume $\Psi$ is of the form

$$
\Psi(v)=-\lambda-\delta v-\int_{0}^{\infty}\left(1-e^{-v u}\right) \pi(\mathrm{d} u)
$$

with $\delta \geq 0$ and $\int_{0}^{\infty}(1 \wedge u) \pi(\mathrm{d} u)<\infty$. Recall the condition

$$
\text { (A) } \delta=0 \text { and } \bar{\pi}(0)+\lambda \leq c / 2
$$

i) If (A) is satisfied then for all $x \geq 0, \mathbb{P}_{x}\left(\lim _{t \rightarrow \infty} U_{t}^{0}=0\right)=1$.

ii) If (A) is not satisfied then for all $x \geq 0$,

$$
\mathbb{P}_{x}\left(\lim _{t \rightarrow \infty} U_{t}^{0}=0\right)=1-\mathbb{P}_{x}\left(\lim _{t \rightarrow \infty} U_{t}^{0}=\infty\right)=\frac{\int_{x}^{\infty} \exp \left(\int_{\theta}^{y} \frac{2 \Psi(z)}{c z} \mathrm{~d} z\right) \mathrm{d} y}{\int_{0}^{+\infty} \exp \left(\int_{\theta}^{y} \frac{2 \Psi(z)}{c z} \mathrm{~d} z\right) \mathrm{d} y}
$$

\section{Infinity as an entrance boundary}

In all this section, we assume $\mathcal{E}=\infty$ (and thus $\lambda=0$ ). Recall from Lemma 4.5 that it ensures the inaccessibility of $\infty$ for the process $\left(Z_{t}^{\min }, t \geq 0\right)$ and from Lemma 5.2-(1) that 0 is an exit for the $\Psi$-generalized Feller diffusion. In Section 6.1, we proceed to prove Theorem 3.3. It will follow from Lemma 6.1 and Lemma 6.3. In Section 6.2, we establish Corollary 3.6, Theorem 3.7 and Theorem 3.9 in the case $\mathcal{E}=\infty$.

\subsection{Duality and entrance law}

The boundary 0 being an exit, there is no ambiguity at the boundary, and we simply call $\left(U_{t}, t \geq 0\right)$ the $\Psi$-generalized Feller diffusion solution to (5.1).

Lemma 6.1 (Duality lemma). Assume $\mathcal{E}=\infty$. For all $z \in[0, \infty)$ and $x \in(0, \infty)$, the following duality holds

$$
\mathbb{E}_{z}\left[e^{-x Z_{t}^{\min }}\right]=\mathbb{E}_{x}\left[e^{-z U_{t}}\right]
$$

Proof. Recall $\left(R_{t}, t \geq 0\right)$ the Ornstein-Uhlenbeck type process. For any $x>0$, by Itô's formula, one sees that the process

$$
\left(e^{-x R_{t}}-\int_{0}^{t} \mathscr{L}^{R} e_{x}\left(R_{s}\right) \mathrm{d} s, t \geq 0\right)
$$


is a local martingale. Recall $t \mapsto C_{t}$, the process

$$
\left(M_{t}^{Z^{\min }}, t \geq 0\right):=\left(e^{-x Z_{t}^{\min }}-\int_{0}^{t} \mathscr{L} e_{x}\left(Z_{s}^{\min }\right) \mathrm{d} s, t \geq 0\right)
$$

satisfies $\left(M_{t}^{Z^{\min }}, t \geq 0\right)=\left(M_{C_{t}}^{R}, t \geq 0\right)$ and is therefore a local martingale. Since $x>0$, we have that $z \mapsto \mathscr{L} e_{x}(z)$ is bounded and thus $\left(M_{t}^{Z^{\min }}, t \geq 0\right)$ is a martingale. Consider now $\left(U_{t}, t \geq 0\right)$ a $\Psi$-generalized Feller diffusion independent of $\left(Z_{t}^{\min }, t \geq 0\right)$. Similarly, by applying Itô's formula, we have that for any $z \in[0, \infty)$,

$$
\left(M_{t}^{U}, t \geq 0\right):=\left(e^{-z U_{t}}-\int_{0}^{t} \mathscr{A} e_{z}\left(U_{s}\right) \mathrm{d} s, t \geq 0\right)
$$

is a martingale. Recall the generator duality in Lemma $5.1, \mathscr{A} e_{z}(x)=\mathscr{L} e_{x}(z)$ and set $g(z, x):=\mathscr{L} e_{x}(z)$. We apply Ethier-Kurtz's duality result [EK86, Corollary 4.15 page 196] (in their notation $\left.\alpha=\beta=0, \tau=\infty, \sigma=\tau_{\epsilon}\right)$ to the process $\left(Z_{t}^{\min }, t \geq 0\right)$ and the stopped process $\left(U_{t \wedge \tau_{\epsilon}}, t \geq 0\right)$ at time $\tau_{\epsilon}:=\inf \left\{t \geq 0, U_{t} \leq \epsilon\right\}$. For the corollary to be applicable, an integrability condition, called (4.50) in [EK86] is required. We will verify this condition after and shall see the role played by $\tau_{\epsilon}$. We obtain, for $x \geq \epsilon$

$$
\begin{aligned}
\mathbb{E}_{z}\left[e^{-x Z_{t}^{\min }}\right]-\mathbb{E}_{x}\left[e^{-z U_{t \wedge \tau_{\epsilon}}}\right] & =\int_{0}^{t} \mathbb{E}\left[\mathbb{1}_{t-s>\tau_{\epsilon}} g\left(Z_{s}^{\min }, U_{(t-s) \wedge \tau_{\epsilon}}\right)\right] \mathrm{d} s \\
& =\mathbb{E}\left[\int_{0}^{t-\tau_{\epsilon} \wedge t} \mathscr{L} e_{\epsilon}\left(Z_{s}^{\min }\right) \mathrm{d} s\right] .
\end{aligned}
$$

Since the process $t \mapsto e^{-\epsilon Z_{t}^{\min }}-\int_{0}^{t} \mathscr{L} e_{\epsilon}\left(Z_{s}^{\min }\right) \mathrm{d} s$ is a martingale and $\tau_{\epsilon}$ is independent of $\left(Z_{t}^{\min }, t \geq 0\right)$, we see from the last equality that

$$
\mathbb{E}_{x}\left[e^{-z U_{t \wedge \tau_{\epsilon}}}\right]-\mathbb{E}_{z}\left[e^{-x Z_{t}^{\min }}\right]=-\mathbb{E}\left[\int_{0}^{t-\tau_{\epsilon} \wedge t} \mathscr{L} e_{\epsilon}\left(Z_{s}^{\min }\right) \mathrm{d} s\right]=e^{-\epsilon z}-\mathbb{E}_{z}\left[e^{\left.-\epsilon Z_{t-t \wedge \tau_{\epsilon}}^{\min }\right]} .\right.
$$

By letting $\epsilon$ to $0, \tau_{\epsilon} \underset{\epsilon \rightarrow 0}{\longrightarrow} \tau_{0}$ a.s and the last equality provides

$$
\mathbb{E}_{x}\left[e^{-z U_{t \wedge \tau_{\epsilon}}}\right]-\mathbb{E}_{z}\left[e^{-x Z_{t}^{\min }}\right] \underset{\epsilon \rightarrow 0}{\longrightarrow} 1-\mathbb{P}_{z}\left(Z_{t-t \wedge \tau_{0}}^{\min }<\infty\right) .
$$

By Lemma 4.5, we know that under the condition $\mathcal{E}=\infty$ the process $\left(Z_{t}^{\min }, t \geq 0\right)$ does not explode. Therefore the limit above is 0 and $\mathbb{E}_{z}\left[e^{-x Z_{t}^{\min }}\right]=\mathbb{E}_{x}\left[e^{-z U_{t \wedge \tau_{0}}}\right]$ for all $x>0$ and $z \in[0, \infty)$. On the other hand, by Lemma 5.2, under the condition $\mathcal{E}=\infty, 0$ is an exit of the diffusion and thus

$$
\mathbb{E}_{z}\left[e^{-x Z_{t}^{\min }}\right]=\mathbb{E}_{x}\left[e^{-z U_{t}}\right]
$$

It remains to verify the technical condition (4.50) in Ethier-Kurtz [EK86] page 192. Namely, for any $T>0$ and $\epsilon>0$ fixed, we need to show that the random variables $\sup _{s, t \leq T} \exp \left(-U_{s \wedge \tau_{\epsilon}} Z_{t}^{\min }\right)$ and

$$
\sup _{s, t \leq T}\left|g\left(Z_{t}^{\min }, U_{s \wedge \tau_{\epsilon}}\right)\right|, \text { where } g(z, u)=\Psi(u) z e^{-u z}+\frac{c}{2} u z^{2} e^{-u z}
$$

are integrable. Since $\sup _{s, t \leq T} \exp \left(-U_{s \wedge \tau_{\epsilon}} Z_{t}^{\text {min }}\right)$ is clearly bounded by 1 , we only need to focus on $\sup _{s, t \leq T}\left|g\left(Z_{t}^{\text {min }}, U_{s \wedge \tau_{\epsilon}}\right)\right|$. The stopping time $\tau_{\epsilon}$ will play an important role at this stage. For any mechanism $\Psi$, if $u \geq \epsilon>0$, then $|\Psi(u)| \leq b_{\epsilon} u^{2}$ for some $b_{\epsilon}>0$. Recall 
$x \geq \epsilon>0$. Since for all $s \geq 0, U_{s \wedge \tau_{\epsilon}} \geq \epsilon$ a.s. under $\mathbb{P}_{x}, u z^{2} e^{-u z} \leq \frac{2}{u}$ and $z e^{-\epsilon z} \leq \frac{1}{\epsilon}$ for any $u>0$ and $z>0$, we have that

$$
\begin{aligned}
\left|g\left(Z_{t}^{\min }, U_{s \wedge \tau_{\epsilon}}\right)\right| & =\left|\Psi\left(U_{s \wedge \tau_{\epsilon}}\right) Z_{t}^{\min } e^{-U_{s \wedge \tau_{\epsilon}} Z_{t}^{\min }}+\frac{c}{2} U_{s \wedge \tau_{\epsilon}}\left(Z_{t}^{\min }\right)^{2} e^{-U_{t \wedge \tau_{\epsilon}} Z_{t}^{\min }}\right| \\
& \leq b_{\epsilon} U_{s \wedge \tau_{\epsilon}}^{2} Z_{t}^{\min } e^{-U_{s \wedge \tau_{\epsilon}} Z_{t}^{\min }}+\frac{c}{2} U_{s \wedge \tau_{\epsilon}}\left(Z_{t}^{\min }\right)^{2} e^{-U_{s \wedge \tau_{\epsilon}} Z_{t}^{\min }} \\
& \leq b_{\epsilon} U_{s \wedge \tau_{\epsilon}}^{2} Z_{t}^{\min } e^{-\epsilon Z_{t}^{\min }}+\frac{c}{U_{s \wedge \tau_{\epsilon}}} \\
& \leq \frac{b_{\epsilon}}{\epsilon} U_{s \wedge \tau_{\epsilon}}^{2}+\frac{c}{\epsilon}
\end{aligned}
$$

We now argue by comparison in order to show that $\sup _{s \leq T} U_{s \wedge \tau_{\epsilon}}^{2}$ is integrable. Since $\Psi$ is convex and $\Psi(0) \leq 0$, the map $u \mapsto \frac{\Psi(u)}{u}$ is nondecreasing and when $u \geq \epsilon$, we have $\Psi(u) \geq \frac{\Psi(\epsilon)}{\epsilon} u \geq-\gamma_{\epsilon} u$ for some $\gamma_{\epsilon}>0$. Recall that $\Psi$ is locally Lipschitz on $(0, \infty)$. Applying the results of [RY99, Section 3, Chapter IX], one can then construct, with the same Brownian motion $\left(B_{t}, t \geq 0\right)$, the process $\left(U_{t}, t \geq 0\right)$ as a strong solution to (5.1) with 0 exit and the process $\left(V_{t}, t \geq 0\right)$ as a strong solution to

$$
\mathrm{d} V_{t}=\sqrt{c V_{t}} \mathrm{~d} B_{t}+\gamma_{\epsilon} V_{t} \mathrm{~d} t, \quad V_{0}=x .
$$

Both processes are adapted to the natural filtration of $\left(B_{t}, t \geq 0\right)$. Applying the comparison theorem [RY99, Theorem IX.3.7] up to the stopping time $\tau_{\epsilon}$, one has that almost surely for any $0 \leq s \leq \tau_{\epsilon}, U_{s} \leq V_{s}$. Note that $\left(V_{t}, t \geq 0\right)$ is a supercritical Feller diffusion with branching mechanism $\Phi(u)=\frac{c}{2} u^{2}+\gamma_{\epsilon} u$. It is easily checked that for any $t \geq 0$, $V_{t}$ has a second moment. Moreover, the process $\left(V_{s}, s \geq 0\right)$ is a submartingale and by Doob's inequality

$$
\mathbb{E}_{x}\left[\sup _{s \leq T} V_{s}^{2}\right] \leq 4 \mathbb{E}_{x}\left[V_{T}^{2}\right]<\infty .
$$

Since for any $\epsilon>0, \sup _{s \leq T \wedge \tau_{\epsilon}} U_{s}^{2} \leq \sup _{s \leq T} V_{s}^{2}$, the proof is complete.

Let $\left(P_{t}^{\min }, t \geq 0\right)$ be the semigroup of $\left(Z_{t}^{\min }, t \geq 0\right)$. Lemma 4.5 ensures that when $\mathcal{E}=\infty, \infty$ is inaccessible. To see that $\infty$ is an entrance boundary, we show in the following lemmas how to define a Feller semigroup coinciding with $\left(P_{t}^{\min }, t \geq 0\right)$ over $[0, \infty)$, with an entrance law from $\infty$.

Lemma 6.2. For any $t>0, x \mapsto \mathbb{P}_{x}\left(U_{t}=0\right)$ is the Laplace transform of a certain probability measure $\eta_{t}$ over $[0, \infty)$. Moreover $\eta_{t} \rightarrow \eta_{0}:=\delta_{\infty}$ weakly as $t \rightarrow 0$.

Proof. By taking limits as $z \rightarrow \infty$ in the duality formula in Lemma 6.1, one has:

$$
\lim _{z \rightarrow \infty} \mathbb{E}_{z}\left(e^{-x Z_{t}^{\min }}\right)=\lim _{z \rightarrow \infty} \mathbb{E}_{x}\left(e^{-z U_{t}}\right)=\mathbb{P}_{x}\left(U_{t}=0\right) .
$$

Since 0 is an exit thanks to the assumption $\mathcal{E}=\infty, \mathbb{P}_{x}\left(U_{t}=0\right)=\mathbb{P}_{x}\left(\tau_{0} \leq t\right)>0$. Moreover, $\lim _{x \rightarrow 0} \mathbb{P}_{x}\left(\tau_{0} \leq t\right)=\mathbb{P}_{0+}\left(\tau_{0} \leq t\right)=1$. Therefore, by Lévy's continuity theorem, $x \mapsto \mathbb{P}_{x}\left(\tau_{0} \leq t\right)$ is the Laplace transform of a certain probability measure $\eta_{t}$ on $[0, \infty)$. This measure $\eta_{t}$ is the weak limit of the law of $Z_{t}^{\min }$ under $\mathbb{P}_{z}$ as $z \rightarrow \infty$. By continuity of the paths of $\left(U_{t}, t \geq 0\right)$, if $x>0$, then $\lim _{t \rightarrow 0} \mathbb{P}_{x}\left(U_{t}=0\right)=0$, and if $x=0$ then $\lim _{t \rightarrow 0} \mathbb{P}_{x}\left(U_{t}=0\right)=1$. This entails that $\eta_{t} \rightarrow \delta_{\infty}$ weakly as $t \rightarrow 0$.

From now on, we will work with the following definition of $e_{x}$ over $[0, \infty]$. For any $x>0, e_{x}(z)=e^{-x z}$ for all $z \in[0, \infty]$ and $e_{0}(z)=1$ for all $z \in[0, \infty]$. Note that $e_{0+}(z):=\lim _{x \rightarrow 0 x>0} e_{x}(z)=\mathbb{1}_{\{z<\infty\}}$. 
Lemma 6.3. For any function $f \in C_{b}([0, \infty])$ and any $t \geq 0$, set $P_{t} f(z):=P_{t}^{\min } f(z)$ for any $z \in[0, \infty)$ and $P_{t} f(\infty):=\int_{0}^{\infty} f(u) \eta_{t}(\mathrm{~d} u)$. This defines a Feller semigroup $\left(P_{t}, t \geq 0\right)$ over $[0, \infty]$. Furthermore, if $\left(Z_{t}, t \geq 0\right)$ is a càdlàg Markov process with semigroup $\left(P_{t}, t \geq 0\right)$, and $T:=\inf \left\{t>0 ; Z_{t}<\infty\right\}$, then $\mathbb{P}_{\infty}(T=0)=1$.

Proof. The subalgebra generated by the maps $\left\{e_{x}(\cdot), x \geq 0\right\}$ is separating $[0, \infty]$ (recall that $e_{0}(z)=1$ for any $\left.z \in[0, \infty]\right)$ and therefore by the Stone-Weierstrass theorem, is dense in $C_{b}([0, \infty])$ for the supremum norm. By Lemma 6.1, for any $x \geq 0, P_{t} e_{x}(z)=\mathbb{E}_{x}\left[e^{-z U_{t}}\right]$ when $z \in[0, \infty)$. The map $z \mapsto P_{t} e_{x}(z)$ is therefore continuous on $[0, \infty)$. The continuity at $z=\infty$ holds since according to Lemma 6.2, $P_{t} e_{x}(\infty)=\lim _{z \rightarrow \infty} P_{t} e_{x}(z)$. By StoneWeierstrass theorem, $z \mapsto P_{t} f(z)$ is continuous on $[0, \infty]$ with any $f \in C_{b}([0, \infty])$. Hence $P_{t} C_{b}([0, \infty]) \subset C_{b}([0, \infty])$. We show now that $\left(P_{t}, t \geq 0\right)$ is a semigroup. Since it coincides with the semigroup $\left(P_{t}^{\min }, t \geq 0\right)$ on $[0, \infty)$, we have that for any $s, t \geq 0$, any function $f \in C_{b}([0, \infty])$ and any $z \in[0, \infty) P_{t+s} f(z)=P_{t} P_{s} f(z)$. For $z=\infty$, we have

$$
P_{t+s} f(\infty)=\lim _{z \rightarrow \infty} P_{t+s} f(z)=\lim _{z \rightarrow \infty} P_{t} P_{s} f(z)=\int P_{s} f(y) \eta_{t}(\mathrm{~d} y) .
$$

The last equality above holds since $P_{s} f \in C_{b}([0, \infty])$. This provides $P_{t+s} f(\infty)=P_{t} P_{s} f(\infty)$. It remains to justify the continuity of $\left(P_{t}, t \geq 0\right)$ at 0 . That is to say $P_{t} f(z) \underset{t \rightarrow 0}{\longrightarrow} f(z)$ for any $z \in[0, \infty]$ and any $f \in C_{b}([0, \infty])$. Since $\left(U_{t}, t \geq 0\right)$ is a diffusion (with continuous paths), the map $t \mapsto P_{t} e_{x}(z)=\mathbb{E}_{x}\left(e^{-z U_{t}}\right)$ is continuous, in particular continuous at 0 . Hence, $t \mapsto P_{t} f(z)$ is continuous at zero for any $z \in[0, \infty)$. For $z=\infty$, since $\eta_{t} \underset{t \rightarrow 0}{\longrightarrow} \delta_{\infty}$ weakly, $P_{t} f(\infty) \underset{t \rightarrow 0}{\longrightarrow} f(\infty)$. This entails the Feller property of $\left(P_{t}, t \geq 0\right)$ (see e.g. [RY99, Section 2, Chapter III]), which ensures the existence of a Markov process $\left(Z_{t}, t \geq 0\right)$ with semigroup $\left(P_{t}, t \geq 0\right)$ and càdlàg paths. We show now that $\infty$ is instantaneous. According to Lemma 6.2 for every $t>0, \eta_{t}$ is a probability measure over $\mathbb{R}_{+}$. Thus $\mathbb{P}_{\infty}(T<t)=\mathbb{P}_{\infty}\left(Z_{t}<\infty\right)=\eta_{t}\left(\mathbb{R}_{+}\right)=1$. Letting $t$ to 0 provides $\mathbb{P}_{\infty}(T=0)=1$.

The proof of Theorem 3.3 is obtained by combining Lemma 6.1 and Lemma 6.3.

We give in the next lemma an alternative proof for the property of entrance at $\infty$, based on arguments that do not involve duality.

Lemma 6.4. Define $\zeta_{a}:=\inf \left\{t \geq 0 ; Z_{t}^{\min } \leq a\right\}$ for any $a \geq 0$. For any large enough positive $a$, one has $\sup _{z \geq a} \mathbb{E}_{z}\left(\zeta_{a}\right) \leq \frac{4}{c a}$.

Proof. Recall $\mathscr{G}$ the generator of a CSBP with mechanism $\Psi$. Let $h(z)=\frac{1}{z}$. One has

$$
\begin{aligned}
\mathscr{G} h(z) & =\frac{\sigma^{2}}{z^{2}}+\frac{\gamma}{z}+\int_{0}^{\infty} z\left(\frac{1}{z+h}-\frac{1}{z}+\mathbb{1}_{\{h \leq 1\}} h \frac{1}{z^{2}}\right) \pi(\mathrm{d} h) \\
& =\frac{\sigma^{2}}{z^{2}}+\frac{\gamma}{z}-\int_{1}^{\infty} \frac{h}{z+h} \pi(\mathrm{d} h)+\int_{0}^{1} h\left(\frac{1}{z}-\frac{1}{z+h}\right) \pi(\mathrm{d} h) .
\end{aligned}
$$

By Lebesgue's theorem, $\mathscr{G} h(z) \underset{z \rightarrow \infty}{\longrightarrow} 0$. Since $\mathscr{L} h(z)=\mathscr{G} h(z)+\frac{c}{2}$, there exists $a>0$ such that for all $z \geq a, \mathscr{L} h(z) \geq \frac{c}{4}$. Since by assumption, $\mathcal{E}=\infty$, the process $\left(Z_{t}^{\min }, t \geq 0\right)$ does not explode and there exists a localizing sequence of stopping times $\left(T_{m}, m \geq 1\right)$ such that $T_{m} \underset{m \rightarrow \infty}{\longrightarrow} \infty$ almost surely and $\left(M_{t \wedge T_{m}}, t \geq 0\right)$ is a bounded martingale, where

$$
M_{t}=h\left(Z_{t}^{\min }\right)-\int_{0}^{t} \mathscr{L} h\left(Z_{s}^{\min }\right) \mathrm{d} s
$$


By the optional stopping theorem, $\mathbb{E}_{z}\left[M_{\zeta_{a} \wedge T_{m}}\right]=h(z)$ and we obtain, letting $m$ to $\infty$

$$
\mathbb{E}_{z}\left[h\left(Z_{\zeta_{a}}^{\min }\right)\right]-h(z)=\frac{1}{a}-\frac{1}{z}=\mathbb{E}_{z}\left[\int_{0}^{\zeta_{a}} \mathscr{L} h\left(Z_{s}^{\mathrm{min}}\right) \mathrm{d} s\right] \geq \frac{c}{4} \mathbb{E}_{z}\left(\zeta_{a}\right) .
$$

We conclude that $\mathbb{E}_{z}\left(\zeta_{a}\right) \leq \frac{4}{c}\left(\frac{1}{a}-\frac{1}{z}\right)$ for any $z \geq a$. The entrance property can be deduced by following the proof of Kallenberg [Kal02, Theorem 23.13].

\subsection{Longterm behavior and stationarity}

We now show Corollary 3.6, Theorem 3.7 and Theorem 3.9 in the case $\mathcal{E}=\infty$.

Lemma 6.5 (Corollary 3.6: 0 as exit or natural boundary). Let $\zeta_{0}:=\inf \left\{t>0 ; Z_{t}=0\right\}$. If $\int^{\infty} \frac{\mathrm{d} u}{\Psi(u)}<\infty$ then for any $z \geq 0, \mathbb{P}_{z}\left(\zeta_{0}<\infty\right)>0$ and 0 is an exit. If $\int^{\infty} \frac{\mathrm{d} u}{\Psi(u)}=\infty$ then for any $z>0, \mathbb{P}_{z}\left(\zeta_{0}=\infty\right)=1$ and 0 is natural.

Proof. By Lemma 6.1 and Lemma 6.3, for all $z \in[0, \infty]$ and $x \in(0, \infty)$,

$$
\mathbb{E}_{z}\left[e^{-x Z_{t}}\right]=\mathbb{E}_{x}\left[e^{-z U_{t}}\right] .
$$

According to Lemma 5.2-2), for any $t \geq 0, U_{t}<\infty, \mathbb{P}_{x}$-almost surely. Therefore

$$
\lim _{z \rightarrow 0+} \mathbb{E}_{z}\left[e^{-x Z_{t}}\right]=\mathbb{P}_{x}\left(U_{t}<\infty\right)=1
$$

and 0 is either an exit or a natural boundary. For any $z>0$,

$$
\mathbb{P}_{z}\left(\zeta_{0} \leq t\right)=\lim _{x \rightarrow \infty} \mathbb{E}_{z}\left[e^{-x Z_{t}}\right]=\lim _{x \rightarrow \infty} \mathbb{E}_{x}\left[e^{-z U_{t}}\right]=\mathbb{E}_{\infty}\left[e^{-z U_{t}}\right] .
$$

Therefore $\mathbb{P}_{z}\left(\zeta_{0} \leq t\right)>0$ if and only if $\mathbb{E}_{\infty}\left[e^{-z U_{t}}\right]>0$. By Lemma 5.2-2), $\infty$ is an entrance boundary of the diffusion $\left(U_{t}, t \geq 0\right)$ if and only if $\int^{\infty} \frac{\mathrm{d} u}{\Psi(u)}<\infty$.

Lemma 6.6 (Theorem 3.7: stationarity). If the assumption (A) (with $\lambda=0$ ) is not satisfied then for all $x \geq 0$

$$
\mathbb{E}_{z}\left(e^{-x Z_{t}}\right) \underset{t \rightarrow \infty}{\longrightarrow} L(x):=\frac{\int_{x}^{\infty} \exp \left(\int_{\theta}^{y} \frac{2 \Psi(z)}{c z} \mathrm{~d} z\right) \mathrm{d} y}{\int_{0}^{\infty} \exp \left(\int_{\theta}^{y} \frac{2 \Psi(z)}{c z} \mathrm{~d} z\right) \mathrm{d} y} .
$$

Moreover $L$ is the Laplace transform of a probability measure supported on $\left(\frac{2 \delta}{c}, \infty\right)$, where $\delta=-\lim _{u \rightarrow \infty} \frac{\Psi(u)}{u}$. If the assumption (A) is satisfied then for all $x \geq 0$,

$$
\mathbb{E}_{z}\left(e^{-x Z_{t}}\right) \underset{t \rightarrow \infty}{\longrightarrow} 1 .
$$

Proof. According to Lemma 5.3, $\left(U_{t}, t \geq 0\right)$ converges almost surely towards either 0 or $\infty$ as $t$ goes to $\infty$. Thus, for any $z \in(0, \infty]$ and $x \geq 0$,

$$
\mathbb{E}_{z}\left(e^{-x Z_{t}}\right)=\mathbb{E}_{x}\left(e^{-z U_{t}} \mathbb{1}_{\left\{\lim _{t \rightarrow \infty} U_{t}=0\right\}}+e^{-z U_{t}} \mathbb{1}_{\left\{\lim _{t \rightarrow \infty} U_{t}=\infty\right\}}\right) \underset{t \rightarrow \infty}{\longrightarrow} \mathbb{P}_{x}\left(\lim _{t \rightarrow \infty} U_{t}=0\right) .
$$

A direct application of Lemma 5.3 provides the two stated convergences. The map $L$ is clearly continuous at 0 and by Lévy's continuity theorem, $L$ is the Laplace transform of a certain probability measure. The support of the stationary measure is $\left(\frac{2 \delta}{c}, \infty\right)$ since $\left(Z_{t}^{\min }, t \geq 0\right)$ is irreducible in $\left(\frac{2 \delta}{c}, \infty\right)$, see the Remark below Lemma 4.6.

The next Lemma establishes part 1) of Theorem 3.9 under the additional condition $\mathcal{E}=\infty$. We get the same part proved under $\mathcal{E}<\infty$ in the next section. 
Lemma 6.7. Assume $\Psi(z) \geq 0$ for some $z>0$ then

1) If $\int^{\infty} \frac{\mathrm{d} u}{\Psi(u)}=\infty$, then $Z_{t}>0$ for any $t \geq 0$ a.s. and $Z_{t} \underset{t \rightarrow \infty}{\longrightarrow} 0$ a.s.

2) If $\int^{\infty} \frac{\mathrm{d} u}{\Psi(u)}<\infty$, then $\left(Z_{t}, t \geq 0\right)$ gets absorbed at 0 in finite time almost surely.

Proof. Note first that Lemma 4.3 ensures that $Z_{t} \underset{t \rightarrow \infty}{\longrightarrow} 0$ a.s. If $\int^{\infty} \frac{\mathrm{d} z}{\Psi(z)}=\infty$, we have seen in Lemma 6.5 that 0 is inaccessible. Assume now $\int^{\infty} \frac{\mathrm{d} z}{\Psi(z)}<\infty$, then by Lemma 5.3-1) and (6.1), we have $\mathbb{P}_{z}\left(\zeta_{0} \leq t\right)=\mathbb{E}_{\infty}\left(e^{-z U_{t}}\right) \underset{t \rightarrow \infty}{\longrightarrow} 1$, thus $\mathbb{P}_{z}\left(\zeta_{0}<\infty\right)=1$.

\section{Infinity as regular reflecting or exit boundary}

In this section, we assume $\mathcal{E}<\infty$. Recall that we have seen in Lemma 5.2 that if $0 \leq 2 \lambda / c<1$ then 0 is a regular boundary of the diffusion (5.1) and if $2 \lambda / c \geq 1$, then 0 is an entrance boundary. We will prove Theorems 3.4 and 3.5. The proof of Theorem 3.4 is obtained by combining Lemmas 7.2, 7.4, 7.5. Theorem 3.5 follows from Lemmas 7.3, 7.4 and 7.6.

Recall $\Psi_{k}$ the branching mechanism associated to the triplet $\left(\sigma, \gamma, \pi_{k}\right)$ with $\pi_{k}(\mathrm{~d} u)=$ $\pi_{\mid(0, k)}(\mathrm{d} u)+(\bar{\pi}(k)+\lambda) \delta_{k}$, that is

$$
\Psi_{k}(x)=\frac{\sigma^{2}}{2} x^{2}-\gamma x+\int_{(0, k)}\left(e^{-x u}-1+x u 1_{u \in(0,1)}\right) \pi(\mathrm{d} u)+\left(e^{-x k}-1\right)(\bar{\pi}(k)+\lambda) .
$$

Note that for all $k \geq 0,\left|\Psi_{k}^{\prime}(0+)\right|<\infty$ and for all $x>0, \Psi_{k}(x) \underset{k \rightarrow \infty}{\longrightarrow} \Psi(x)$. The main idea is to study the logistic $\operatorname{CSBP}\left(Z_{t}^{(k)}, t \geq 0\right)$ with mechanism $\Psi_{k}$ through its dual diffusion, say $\left(U_{t}^{(k)}, t \geq 0\right)$, by invoking Lemma 6.1 . We start by studying the sequence of diffusion processes $\left(U_{t}^{(k)}, t \geq 0\right)$ as $k$ goes to $\infty$. Recall that when $0 \leq 2 \lambda / c<1$, we denote by $\left(U_{t}^{0}, t \geq 0\right)$ the diffusion (5.1) with 0 regular absorbing.

Lemma 7.1. For any $k \geq 0$, denote $\left(U_{t}^{(k)}, t \geq 0\right)$ the unique solution to

$$
\mathrm{d} U_{t}^{(k)}=\sqrt{c U_{t}^{(k)}} \mathrm{d} B_{t}-\Psi_{k}\left(U_{t}^{(k)}\right) \mathrm{d} t .
$$

There exists a probability space on which, with probability 1:

$$
U_{t}^{(k)} \leq U_{t}^{(k+1)}, \text { for all } k \geq 1 \text { and } t \geq 0 .
$$

1) If $0 \leq \frac{2 \lambda}{c}<1$, then as $k$ goes to $\infty$, the sequence of processes $\left(U_{t}^{(k)}, t \geq 0\right)$ converges pointwise almost surely towards a process $\left(U_{t}^{(\infty)}, t \geq 0\right)$ with the same law as $\left(U_{t}^{0}, t \geq 0\right)$ the solution to (5.1) absorbed at 0.

2) If $\frac{2 \lambda}{c} \geq 1$, then as $k$ goes to $\infty$, the sequence of processes $\left(U_{t}^{(k)}, t \geq 0\right)$ converges almost surely towards a process $\left(U_{t}^{(\infty)}, t \geq 0\right)$ with the same law as $\left(U_{t}, t \geq 0\right)$ the solution to (5.1) with 0 entrance.

Proof. One first checks that for any $k \geq 0$ and $x \geq 0, \Psi_{k}(x) \geq \Psi_{k+1}(x)$. Plainly

$$
\begin{aligned}
& \Psi_{k+1}(x)-\Psi_{k}(x) \\
& =\int_{[k, k+1)}\left(e^{-x u}-1\right) \pi(\mathrm{d} u)+\left(e^{-x(k+1)}-1\right)(\bar{\pi}(k+1)+\lambda)-\left(e^{-x k}-1\right)(\bar{\pi}(k)+\lambda) \\
& \leq\left(e^{-x k}-1\right)(\bar{\pi}(k)-\bar{\pi}(k+1))+\left(e^{-x(k+1)}-1\right)(\bar{\pi}(k+1)+\lambda)-\left(e^{-x k}-1\right)(\bar{\pi}(k)+\lambda) \\
& =\left(e^{-x(k+1)}-e^{-x k}\right)(\bar{\pi}(k+1)+\lambda) \leq 0 .
\end{aligned}
$$


Since $\left|\Psi_{k}^{\prime}(0+)\right|<\infty$ for any $k \geq 0$, the branching mechanisms $\Psi_{k}$ are locally Lipschitz on $[0, \infty)$ and therefore by applying the comparison theorem for SDEs ([RY99, Theorem IX.3.7]), one has on some probability space

$$
\mathbb{P}_{x}\left(U_{t}^{(k+1)} \geq U_{t}^{(k)} \text { for all } t \geq 0 \text { and all } k \geq 0\right)=1 .
$$

The existence of the limiting process $\left(U_{t}^{(\infty)}, t \geq 0\right.$ ) (in a pointwise sense) is ensured by monotonicity. We now establish 1) and 2). Set $\tau^{k}:=\inf \left\{t \geq 0, U_{t}^{(k)} \notin(0, \infty)\right\}$ and $\tau^{\infty}:=\inf \left\{t \geq 0, U_{t}^{(\infty)} \notin(0, \infty)\right\}$. Let $\mathscr{A}^{(k)}$ be the generator of $\left(U_{t}^{(k)}, t \geq 0\right)$ and recall $\mathscr{A}$ defined in (5.3). The diffusive part in $\mathscr{A}^{(k)}$ and $\mathscr{A}$ are the same, therefore for any $g \in C_{c}^{2}((0, \infty))$ :

$$
\begin{aligned}
\left\|\mathscr{A}^{(k)} g-\mathscr{A} g\right\|_{\infty} & =\sup _{x \in[0, \infty)}\left|\left(\Psi(x)-\Psi_{k}(x)\right) g^{\prime}(x)\right| \\
& =\sup _{x \in[0, \infty)}\left|\left(-\lambda+\int_{[k, \infty]}\left(e^{-x u}-1\right) \pi(\mathrm{d} u)+\left(1-e^{-x k}\right)(\bar{\pi}(k)+\lambda)\right) g^{\prime}(x)\right| \\
& \leq 2 \sup _{x \in[0, \infty)}\left|e^{-x k} \bar{\pi}(k) g^{\prime}(x)\right|+\lambda \sup _{x \in[0, \infty)}\left|e^{-x k} g^{\prime}(x)\right| .
\end{aligned}
$$

Note that $g^{\prime}$ has compact support in $(0, \infty)$. Since for all $k \geq 1, \bar{\pi}(k) \leq \bar{\pi}(1)$ and for any $x>0, \lim _{k \rightarrow \infty} e^{-x k}=0$, we have that $\left\|\mathscr{A}^{(k)} g-\mathscr{A} g\right\|_{\infty} \underset{k \rightarrow \infty}{\longrightarrow} 0$. Hence, for large enough $k \geq 1,\left\|\mathscr{A}^{(k)} g\right\|_{\infty} \leq 1+\|\mathscr{A} g\|_{\infty}$ and since $U_{s}^{(k)} \underset{k \rightarrow \infty}{\longrightarrow} U_{s}^{(\infty)}$ a.s. for any $s \geq 0$, $\mathscr{A}^{(k)} g\left(U_{s}^{(k)}\right) \underset{k \rightarrow \infty}{\longrightarrow} \mathscr{A} g\left(U_{s}^{(\infty)}\right)$ a.s. for any $s \geq 0$. Let $0 \leq t_{1} \leq t_{2} \leq \cdots \leq t_{n} \leq s<t$ and $f_{1}, \cdots, f_{n}$ some bounded and continuous functions. Since $\left(U_{t}^{(k)}, t \geq 0\right)$ solves (7.1), then the process

$$
t \in\left[0, \tau^{k}\right) \mapsto g\left(U_{t}^{(k)}\right)-\int_{0}^{t} \mathscr{A}^{(k)} g\left(U_{s}^{(k)}\right) \mathrm{d} s
$$

is a martingale. By applying Lebesgue's theorem, we have that

$$
\begin{aligned}
& \mathbb{E}_{z}\left[\left(g\left(U_{t}^{(\infty)}\right)-g\left(U_{s}^{(\infty)}\right)-\int_{s}^{t} \mathscr{A} g\left(U_{r}^{(\infty)}\right) \mathrm{d} r\right) \prod_{i=1}^{n} f_{i}\left(U_{t_{i}}^{(\infty)}\right)\right] \\
& =\lim _{k \rightarrow \infty} \mathbb{E}_{z}\left[\left(g\left(U_{t}^{(k)}\right)-g\left(U_{s}^{(k)}\right)-\int_{s}^{t} \mathscr{A}^{(k)} g\left(U_{r}^{(k)}\right) \mathrm{d} r\right) \prod_{i=1}^{n} f_{i}\left(U_{t_{i}}^{(k)}\right)\right] \\
& =0 .
\end{aligned}
$$

This shows that for any function $g \in \mathcal{C}_{c}^{2}((0, \infty))$, the process

$$
t \in\left[0, \tau^{\infty}\right) \mapsto g\left(U_{t}^{(\infty)}\right)-\int_{0}^{t} \mathscr{A} g\left(U_{s}^{(\infty)}\right) \mathrm{d} s
$$

is a martingale. The process $\left(U_{t}^{(\infty)}, t<\tau^{\infty}\right)$ solves the same martingale problem as $\left(U_{t}, t<\tau\right)$. The latter problem being well-posed (see for instance [Dur96, Section 6.1, Theorem 1.6]), $\left(U_{t}^{(\infty)}, t<\tau^{\infty}\right)$ and $\left(U_{t}, t<\tau\right)$ have the same law.

It remains only to identify the behavior of the process $\left(U_{t}^{(\infty)}, t \geq 0\right)$ after $\tau^{\infty}$. Recall that by Lemma 5.2, $\infty$ is inaccessible, so that $\tau^{\infty}=\inf \left\{t \geq 0, U_{t}^{(\infty)}=0\right\}$ a.s. If $\frac{2 \lambda}{c} \geq 1$ then 0 is an entrance, $\tau^{\infty}=\infty$ a.s. and $\left(U_{t}^{(\infty)}, t \geq 0\right)$ has the same law as $\left(U_{t}, t \geq 0\right)$. If $0 \leq \frac{2 \lambda}{c}<1$, then 0 is regular or exit and $\tau^{\infty}<\infty$ with positive probability. Let $t \geq 0$. On the event $\left\{\tau^{\infty}<\infty\right\}$, by pointwise almost sure convergence, $U_{t+\tau^{\infty}}^{(\infty)}=\lim _{k \rightarrow \infty} U_{t+\tau^{\infty}}^{(k)}$. By 
monotonicity $\tau^{\infty} \geq \tau^{k}$ a.s. for all $k \geq 1$, moreover 0 is an exit for $\left(U_{t}^{(k)}, t \geq 0\right)$ therefore $U_{t+\tau^{\infty}}^{(k)}=U_{t+\tau^{k}}^{(k)}=0$ and $U_{t+\tau^{\infty}}^{(\infty)}=0$ for any $t \geq 0$. We conclude that $\left(U_{t}^{(\infty)}, t \geq 0\right)$ is the diffusion whose generator is $\mathscr{A}$ with 0 absorbing.

The next lemmas will provide proofs of Theorem 3.4 and Theorem 3.5. Recall that for any $k \geq 1,\left(Z_{t}^{(k)}, t \geq 0\right)$ denotes a logistic CSBP with mechanisms $\Psi_{k}$. These processes satisfy $\mathcal{E}_{k}:=\int_{0}^{\theta} \frac{1}{x} \exp \left(\frac{2}{c} \int_{x}^{\theta} \frac{\Psi_{k}(u)}{u} \mathrm{~d} u\right) \mathrm{d} x=\infty$ (since $\left|\Psi_{k}^{\prime}(0+)\right|<\infty$ ) and all results of Section 6 can be applied. According to Lemma 6.3, the processes $\left(Z_{t}^{(k)}, t \geq 0\right)$ have $\infty$ as entrance boundary and are Feller. In the sequel we work with their càdlàg versions.

Lemma 7.2. Assume $\mathcal{E}<\infty$ and $0 \leq \frac{2 \lambda}{c}<1$, the sequence $\left(\left(Z_{t}^{(k)}\right)_{t \geq 0}, k \geq 1\right)$ converges weakly towards a càdlàg Feller process $\left(Z_{t}, t \geq 0\right)$ valued in $[0, \infty]$ such that for all $z \in[0, \infty]$, all $t \geq 0$, and all $x \in[0, \infty)$

$$
\mathbb{E}_{z}\left[e^{-x Z_{t}}\right]=\mathbb{E}_{x}\left[e^{-z U_{t}^{0}}\right]
$$

where $\left(U_{t}^{0}, t \geq 0\right)$ is the $\Psi$-generalized Feller diffusion satisfying (5.1) with 0 regular absorbing.

Proof. Denote by $\left(P_{t}^{(k)}, t \geq 0\right)$ the semi-group of $\left(Z_{t}^{(k)}, t \geq 0\right)$ and $\left(p_{t}^{(k)}(z, \cdot), z \in[0, \infty], t \geq\right.$ 0 ) its transition kernel. Let $t \geq 0$ and $z \in[0, \infty]$ be fixed. For any $k \geq 1$, by Lemma 6.1 , one has for all $x \geq 0, P_{t}^{(k)} e_{x}(z):=\mathbb{E}_{z}\left(e^{-x Z_{t}^{(k)}}\right)=\mathbb{E}_{x}\left(e^{-z U_{t}^{(k)}}\right)$ where $\left(U_{t}^{(k)}, t \geq 0\right)$ is defined in Lemma 7.1. By Lemma 7.1-1), for any $t \geq 0, U_{t}^{(k)}$ converges almost surely towards $U_{t}^{(\infty)}$ as $k$ goes to infinity, and $\left(U_{t}^{(\infty)}, k \geq 1\right)$ has the same law as $\left(U_{t}^{0}, t \geq 0\right)$. Therefore $\lim _{k \rightarrow \infty} \mathbb{E}_{z}\left(e^{-x Z_{t}^{(k)}}\right)=\mathbb{E}_{x}\left(e^{-z U_{t}^{(\infty)}}\right)=\mathbb{E}_{x}\left(e^{-z U_{t}^{0}}\right)$. Therefore $p_{t}^{(k)}(z, \cdot)$ converges weakly as $k$ goes to $\infty$ towards some probability $p_{t}(z, \cdot)$ over $[0, \infty]$ satisfying

$$
\begin{aligned}
P_{t} e_{x}(z) & :=\int_{[0, \infty]} e^{-x y} p_{t}(z, \mathrm{~d} y)=\mathbb{E}_{x}\left[e^{-z U_{t}^{0}}\right] \text { for any } z \in[0, \infty), \\
P_{t} e_{x}(\infty) & :=\int_{[[0, \infty]} e^{-x y} p_{t}(\infty, \mathrm{d} y)=\mathbb{P}_{x}\left(U_{t}^{0}=0\right) .
\end{aligned}
$$

Since $z \in[0, \infty] \mapsto P_{t} e_{x}(z)$ is continuous, then by Stone-Weierstrass theorem, $P_{t} f$ is continuous for any function $f \in C_{b}([0, \infty])$. We stress that at this stage, we do not know that $\left(P_{t}, t \geq 0\right)$ forms a semigroup.

We establish now that for any $x \geq 0,\left(P_{t}^{(k)} e_{x}, k \geq 1\right)$ converges uniformly towards $P_{t} e_{x}$. For any $x \geq 0$, define $\phi_{k}(x, t):=\left\|P_{t}^{(k)} e_{x}-P_{t} e_{x}\right\|_{\infty}$. Recall that $U_{t}^{(k)} \leq U_{t}^{(\infty)}$ for any $t \geq 0, \mathbb{P}_{x}$-almost surely. Therefore, for any $z \in[0, \infty]$ and $t \geq 0$,

$$
\begin{aligned}
& \mathbb{E}_{x}\left[e^{-z U_{t}^{(k)}}-e^{-z U_{t}^{(\infty)}}\right] \\
& =\mathbb{E}_{x}\left[\left(e^{-z U_{t}^{(k)}}-e^{-z U_{t}^{(\infty)}}\right) \mathbb{1}_{\left\{U_{t}^{(k)}<U_{t}^{(\infty)}\right\}}\right] \\
& =\mathbb{E}_{x}\left[\left(e^{-z U_{t}^{(k)}}-e^{-z U_{t}^{(\infty)}}\right) \mathbb{1}_{\left\{0<U_{t}^{(k)}<U_{t}^{(\infty)}\right\}}\right]+\mathbb{E}_{x}\left[\left(e^{-z U_{t}^{(k)}}-e^{-z U_{t}^{(\infty)}}\right) \mathbb{1}_{\left\{U_{t}^{(k)}=0, U_{t}^{(\infty)}>0\right\}}\right] \\
& \leq \mathbb{E}_{x}\left[\left(e^{-z U_{t}^{(k)}}-e^{-z U_{t}^{(\infty)}}\right) \mathbb{1}_{\left\{0<U_{t}^{(k)}<U_{t}^{(\infty)}\right\}}\right]+\mathbb{P}_{x}\left(\tau^{k} \leq t<\tau^{\infty}\right) .
\end{aligned}
$$

Since $\tau^{k} \underset{k \rightarrow \infty}{\longrightarrow} \tau^{\infty}$ a.s. then $\mathbb{P}_{x}\left(\tau^{k} \leq t<\tau^{\infty}\right) \underset{k \rightarrow \infty}{\longrightarrow} 0$. Now, for any $x \geq 0$,

$$
\begin{aligned}
\sup _{z \in[0, \infty]} \mathbb{E}_{x} & {\left[\left(e^{-z U_{t}^{(k)}}-e^{-z U_{t}^{(\infty)}}\right) \mathbb{1}_{\left\{0<U_{t}^{(k)}<U_{t}^{(\infty)}\right\}}\right] } \\
\leq & \mathbb{E}_{x}\left[\sup _{z \in[0, \infty]}\left(e^{-z U_{t}^{(k)}}-e^{-z U_{t}^{(\infty)}}\right) \mathbb{1}_{\left\{0<U_{t}^{(k)}<U_{t}^{(\infty)}\right\}}\right] .
\end{aligned}
$$


The function $z \mapsto e^{-z U_{t}^{(k)}}-e^{-z U_{t}^{(\infty)}}$ reaches its maximum at $z_{k}=\frac{\log U_{t}^{(\infty)}-\log U_{t}^{(k)}}{U_{t}^{(\infty)}-U_{t}^{(k)}}$. Moreover, on the event $\left\{0<U_{t}^{(k)}<U_{t}^{(\infty)}\right\}, z_{k} U_{t}^{(\infty)} \underset{k \rightarrow \infty}{\longrightarrow} 1$ and $z_{k} U_{t}^{(k)} \underset{k \rightarrow \infty}{\longrightarrow} 1$ almost surely, thus by Lebesgue's theorem

$$
\mathbb{E}_{x}\left[\left(e^{-z_{k} U_{t}^{(k)}}-e^{-z_{k} U_{t}^{(\infty)}}\right) \mathbb{1}_{\left\{0<U_{t}^{(k)}<U_{t}^{(\infty)}\right\}}\right] \underset{k \rightarrow \infty}{\longrightarrow} 0 .
$$

Hence, $\phi_{k}(x, t) \underset{k \rightarrow \infty}{\longrightarrow} 0$ and by Stone-Weierstrass theorem, for any $f \in C_{b}([0, \infty]) \| P_{t}^{(k)} f-$ $P_{t} f \|_{\infty} \underset{k \rightarrow \infty}{\longrightarrow} 0$.

As a first consequence of the uniform convergence, we show now that $\left(P_{t}, t \geq 0\right)$ is a semigroup. Let $s, t \geq 0$. Let $g \in C_{b}([0, \infty])$ and $\left(g_{k}, k \geq 1\right)$ such that $g_{k} \underset{k \rightarrow \infty}{\longrightarrow} g$ uniformly. Then,

$$
\begin{aligned}
\left\|P_{t}^{(k)} g_{k}-P_{t} g\right\|_{\infty} & \leq\left\|P_{t}^{(k)} g_{k}-P_{t}^{(k)} g\right\|_{\infty}+\left\|P_{t}^{(k)} g-P_{t} g\right\|_{\infty} \\
& \leq\left\|g_{k}-g\right\|_{\infty}+\left\|P_{t}^{(k)} g-P_{t} g\right\|_{\infty},
\end{aligned}
$$

where we have used in the second inequality that $P_{t}^{(k)}$ is a contraction. The upper bound goes to 0 as $k$ goes to $\infty$ by the uniform convergence. Let $f \in C_{b}([0, \infty])$ and apply the last convergence to $g_{k}:=P_{s}^{(k)} f$ and $g=P_{s} f$, one has, by the semigroup property of $\left(P_{t}^{(k)}, t \geq 0\right)$.

$$
P_{t+s} f=\lim _{k \rightarrow \infty} P_{t+s}^{(k)} f=\lim _{k \rightarrow \infty} P_{t}^{(k)} g_{k}=P_{t} P_{s} f .
$$

Therefore $\left(P_{t}, t \geq 0\right)$ is a semigroup on $C_{b}([0, \infty])$. As in Lemma 6.3 , we see that it is continuous at 0 .

Lastly, since the convergence of semigroups is uniform in $C_{b}([0, \infty])$, one invokes Theorem 2.5 page 167 in [EK86] to claim that the sequence of processes $\left(Z_{t}^{(k)}, t \geq 0\right)$ converges weakly (in the Skorokhod topology) towards a càdlàg Markov process $\left(Z_{t}, t \geq\right.$ $0)$ with semigroup $\left(P_{t}, t \geq 0\right)$.

Lemma 7.3. Assume $\frac{2 \lambda}{c} \geq 1$, the sequence $\left(\left(Z_{t}^{(k)}\right)_{t \geq 0}, k \geq 1\right)$ converges weakly towards a càdlàg Feller process $\left(Z_{t}, t \geq 0\right)$ valued in $[0, \infty]$ such that for all $z \in[0, \infty]$, all $t \geq 0$, and all $x \in(0, \infty)$

$$
\mathbb{E}_{z}\left[e^{-x Z_{t}}\right]=\mathbb{E}_{x}\left[e^{-z U_{t}}\right],
$$

where $\left(U_{t}, t \geq 0\right)$ is the $\Psi$-generalized Feller diffusion (5.1) with 0 entrance.

Proof. The only difference with the proof of Lemma 7.2 lies in the fact that we have to separate the cases $x>0$ and $x=0$, since 0 is an entrance boundary. By Lemma 7.1-2), $\left(U_{t}^{(k)}, t \geq 0\right)$ converges towards $\left(U_{t}^{(\infty)}, t \geq 0\right)$ in a pointwise sense $\mathbb{P}_{x}$-almost surely, for any $x>0$ and $\left(U_{t}^{(\infty)}, t \geq 0\right)$ has the same law as the diffusion $\left(U_{t}, t \geq 0\right)$ solving (5.1) with 0 entrance. The limiting semigroup is then given by $P_{t} e_{x}(z)=\mathbb{E}_{x}\left[e^{-z U_{t}}\right]$ for any $z \in[0, \infty]$ with $x>0$. The case $x=0$ is trivial since for any $z \in[0, \infty]$ and any $k \geq 1$, one has $\mathbb{E}_{z}\left[e^{-0 . Z_{t}^{(k)}}\right]=\mathbb{E}_{0}\left[e^{-z U_{t}^{(k)}}\right]=1$ and so $P_{t} e_{0}(z)=1$ for any $z \in[0, \infty]$. The rest of the proof is similar to the one above. By replacing $\tau^{\infty}$ by $\infty$, since 0 is inaccessible, and using the fact that $\mathbb{P}_{x}\left(\tau^{k} \leq t\right) \underset{k \rightarrow \infty}{\longrightarrow} 0$ for any fixed $t$, we see that $\phi_{k}(x, t) \underset{k \rightarrow \infty}{\longrightarrow} 0$.

The next lemma ensures that both processes $\left(Z_{t}, t \geq 0\right)$ defined above in Lemma 7.2 and Lemma 7.3 are extensions of the minimal logistic CSBP.

Lemma 7.4 (Extension). The limiting processes $\left(Z_{t}, t \geq 0\right)$ in Lemma 7.2 and Lemma 7.3 stopped at $\zeta_{\infty}$ have the same law as $\left(Z_{t}^{\min }, t \geq 0\right)$. 
Proof. By definition, on the event $\left\{\zeta_{\infty}<\infty\right\}$, the process $\left(Z_{t \wedge \zeta_{\infty}}, t \geq 0\right)$ remains at $\infty$ after time $\zeta_{\infty}$. Similarly, we see from the semigroup representation obtained in Lemma 7.2 and Lemma 7.3, that if 0 is reached, then the process $\left(Z_{t}, t \geq 0\right)$ remains at

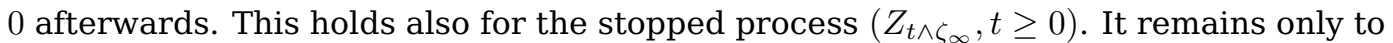
see if $\left(Z_{t \wedge \zeta_{\infty}}, t \geq 0\right)$ satisfies the martingale problem (MP). If this holds true, Lemma 4.2 will ensure that $\left(Z_{t \wedge \zeta_{\infty}}, t \geq 0\right)$ has the same law as $\left(Z_{t}^{\min }, t \geq 0\right)$. Denote by $\mathscr{L}^{(k)}$ the generator of $\left(Z_{t}^{(k)}, t \geq 0\right)$ as defined in Equation (2.5). For any function $f \in C_{c}^{2}((0, \infty))$, one has

$$
\begin{aligned}
\left\|\mathscr{L}^{(k)} f-\mathscr{L} f\right\|_{\infty} & =\sup _{z \in[0, \infty)}\left|f(z+k)(\bar{\pi}(k)+\lambda)-\int_{k}^{\infty} f(z+u) \pi(\mathrm{d} u)\right| \\
& \leq 2(\bar{\pi}(k)+\lambda) \sup _{z \in[k, \infty)}|f(z)| \underset{k \rightarrow \infty}{\longrightarrow} 0 .
\end{aligned}
$$

For any $z \in(0, \infty)$ and any $k \geq 1$, under $\mathbb{P}_{z}$, the process

$$
\left(f\left(Z_{t}^{(k)}\right)-\int_{0}^{t} \mathscr{L}^{(k)} f\left(Z_{s}^{(k)}\right) \mathrm{d} s, t \geq 0\right)
$$

is a martingale. The convergence above being uniform, Lemma 5.1 page 196 in [EK86] entails that the process

$$
\left(f\left(Z_{t}\right)-\int_{0}^{t} \mathscr{L} f\left(Z_{s}\right) \mathrm{d} s, t \geq 0\right)
$$

is a martingale. By [RY99, Corollary III.3.6], the latter process stopped at time $\zeta_{\infty}$,

$$
\left(f\left(Z_{t \wedge \zeta_{\infty}}\right)-\int_{0}^{t \wedge \zeta_{\infty}} \mathscr{L} f\left(Z_{s}\right) \mathrm{d} s, t \geq 0\right)
$$

is a martingale. Now, since $f$ has compact support, for any $s \geq \zeta_{\infty}, \mathcal{L} f\left(Z_{s \wedge \zeta_{\infty}}\right)=0$ and we have that for any $t \geq 0$,

$$
\int_{0}^{t} \mathscr{L} f\left(Z_{s \wedge \zeta_{\infty}}\right) \mathrm{d} s=\int_{0}^{t \wedge \zeta_{\infty}} \mathscr{L} f\left(Z_{s \wedge \zeta_{\infty}}\right) \mathrm{d} s+\int_{t \wedge \zeta_{\infty}}^{t} \mathscr{L} f\left(Z_{s \wedge \zeta_{\infty}}\right) \mathrm{d} s=\int_{0}^{t \wedge \zeta_{\infty}} \mathscr{L} f\left(Z_{s \wedge \zeta_{\infty}}\right) \mathrm{d} s .
$$

We deduce that for any $f \in C_{c}^{2}((0, \infty))$, the process

$$
\left(f\left(Z_{t \wedge \zeta_{\infty}}\right)-\int_{0}^{t} \mathscr{L} f\left(Z_{s \wedge \zeta_{\infty}}\right) \mathrm{d} s, t \geq 0\right)
$$

is a martingale. Therefore the process $\left(Z_{t \wedge \zeta_{\infty}}, t \geq 0\right)$ has the same law as $\left(Z_{t}^{\min }, t \geq 0\right)$. Note that by Lemma 4.5 , since $\mathcal{E}<\infty,\left(Z_{t}^{\min }, t \geq 0\right)$ explodes with a positive probability. This ensures that $\infty$ is accessible for the process $\left(Z_{t}, t \geq 0\right)$.

Lemma 7.5 (Reflecting boundary). Assume $\mathcal{E}<\infty$ and $0 \leq \frac{2 \lambda}{c}<1$. The boundary $\infty$ of the Feller process $\left(Z_{t}, t \geq 0\right)$ is instantaneous regular reflecting. Moreover, $\mathbb{P}_{\infty}$-almost surely, $\left(Z_{t}, t \geq 0\right)$ enters $(0, \infty)$ continuously.

Proof. Recall that we assume $\mathcal{E}<\infty$ and $\frac{2 \lambda}{c}<1$. According to Lemma 7.4 and Lemma $4.5, \infty$ is accessible. Moreover, by applying Lemma 7.2 and Lemma 5.2, one has since $\frac{2 \lambda}{c}<1$, for every $t \geq 0$,

$$
\mathbb{E}_{\infty}\left[e^{-x Z_{t}}\right]=\mathbb{P}_{x}\left(U_{t}^{0}=0\right)=\mathbb{P}_{x}\left(\tau_{0} \leq t\right)>0 .
$$

Thus, $\infty$ is a regular boundary. 
We verify now that $\infty$ is reflecting. Since $\left(U_{t}^{0}, t \geq 0\right)$ has 0 regular absorbing,

$$
\mathbb{P}_{z}\left(Z_{t}<\infty\right)=\lim _{x \rightarrow 0 x>0} \mathbb{E}_{z}\left(e^{-x Z_{t}}\right)=\lim _{x \rightarrow 0 x>0} \mathbb{E}_{x}\left(e^{-z U_{t}^{0}}\right)=\mathbb{E}_{0+}\left(e^{-z U_{t}^{0}}\right)=1 .
$$

This ensures that the Lebesgue measure of the set of times at which the process is at $\infty$ is zero. In other words, $\infty$ is reflecting.

We show now that $\infty$ is instantaneous. Let $T=\inf \left\{t \geq 0, Z_{t}<\infty\right\}$. For any $t>0$, $\mathbb{P}_{\infty}(T \leq t) \geq \mathbb{P}_{\infty}\left(Z_{t}<\infty\right)=1$, then by letting $t$ to 0 , one has $\mathbb{P}_{\infty}(T=0)=1$. By right-continuity of $\left(Z_{t}, t \geq 0\right)$, we have $\mathbb{P}_{\infty}\left(\lim _{t \rightarrow 0} Z_{t}=\infty\right)=1$.

Lemma 7.6 (Exit boundary). Assume $\frac{2 \lambda}{c} \geq 1$. The boundary $\infty$ of the Feller process $\left(Z_{t}, t \geq 0\right)$ is an exit.

Proof. Firstly, by Lemma 7.4 and Lemma 4.5, we know that the process $\left(Z_{t}, t \geq 0\right)$ explodes with positive probability (note that $\lambda>0$, so that by Lemma 4.8, on the event of explosion, it will explode by a jump). Moreover, by Lemma 7.3, letting $z \rightarrow \infty$, we obtain $\mathbb{E}_{\infty}\left[e^{-x Z_{t}}\right]=\mathbb{P}_{x}\left(U_{t}=0\right)=0$ since 0 is an entrance boundary of $U$. Therefore, $\left(Z_{t}, t \geq 0\right)$ gets absorbed at $\infty$.

The proof of Theorem 3.4 now follows by combining Lemmas 7.2, 7.4, 7.5. Theorem 3.5 follows from Lemmas 7.3, 7.4 and 7.6.

In order to understand the long-term behavior of the extended process $\left(Z_{t}, t \geq 0\right)$, we establish now Corollary 3.6, Theorem 3.7 and Theorem 3.9 in the case $\mathcal{E}<\infty$. The arguments for Corollary 3.6 and Theorem 3.7 are exactly the same as those in Lemma 6.5 and Lemma 6.6 for the case $\mathcal{E}=\infty$ (but with $\lambda \geq 0$ ). Indeed, according to Lemma 5.3, the dual diffusion $\left(U_{t}^{0}, t \geq 0\right)$ can leave the interval $(0, \infty)$ by $\infty$ only when the condition (A) is not satisfied. We show Theorem 3.9 in the case $\mathcal{E}<\infty$. The next lemma shows Theorem 3.9-1).

Lemma 7.7. Assume $0 \leq \frac{2 \lambda}{c}<1, \mathcal{E}<\infty$ and $\Psi(z) \geq 0$ for some $z>0$, then $Z_{t} \underset{t \rightarrow \infty}{\longrightarrow} 0$ a.s. If $\int^{\infty} \frac{\mathrm{d} z}{\Psi(z)}=\infty$ then $Z_{t}>0$ for all $t \geq 0$ a.s (the boundary 0 is attracting). If $\int^{\infty} \frac{\mathrm{d} z}{\Psi(z)}<\infty$, then for any $z \in[0, \infty], \mathbb{P}_{z}\left(\zeta_{0}<\infty\right)=1$.

Proof. Let $a>0$. Define $\zeta_{\infty}^{(0)}:=0, \zeta_{a}^{(n)}:=\inf \left\{t>\zeta_{\infty}^{(n-1)} ; Z_{t} \leq a\right\}$ and $\zeta_{\infty}^{(n)}:=\inf \{t>$ $\left.\zeta_{a}^{(n)} ; Z_{t}=\infty\right\}$. By Lemma 5.3-1), since $-\Psi$ is not the Laplace exponent of a subordinator, $U_{t}^{0} \underset{t \rightarrow \infty}{\longrightarrow} 0$ and by Lemma 7.2, $\mathbb{E}_{z}\left[e^{-x Z_{t}}\right] \underset{t \rightarrow \infty}{\longrightarrow} 1$. Therefore $Z_{t} \underset{t \rightarrow \infty}{\longrightarrow} 0$ in probability. Since $\left\{Z_{t} \leq a\right\} \subset\left\{\zeta_{a}^{(1)} \leq t\right\}$ and $\mathbb{P}_{z}\left(Z_{t} \leq a\right) \underset{t \rightarrow \infty}{\longrightarrow} 1$, we have that $\zeta_{a}^{(1)}<\infty$ a.s. Ap-

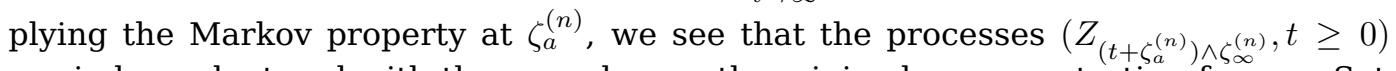
are independent and with the same law as the minimal process starting from $a$. Set

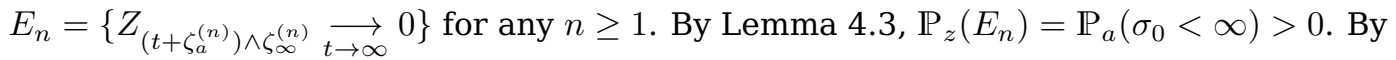
independence, $\mathbb{P}_{z}\left(\bigcap_{n=1}^{\infty} E_{n}^{c}\right)=0$ and therefore $Z_{t} \underset{t \rightarrow \infty}{\longrightarrow} 0$ a.s.

Assume $\int^{\infty} \frac{\mathrm{d} z}{\Psi(z)}=\infty$, since $\infty$ is a natural boundary of $\left(U_{t}^{0}, t \geq 0\right)$ therefore by Lemma 7.2, $\mathbb{P}_{z}\left(\zeta_{0} \leq t\right)=\mathbb{E}_{\infty}\left(e^{-z U_{t}^{0}}\right)=0$ for any $t \geq 0$. Thus $Z_{t}>0$ for all $t \geq 0$ a.s. Now if $\int^{\infty} \frac{\mathrm{d} z}{\Psi(z)}<\infty, \infty$ is an entrance of $\left(U_{t}^{0}, t \geq 0\right)$ and by Lemma 7.2, $\mathbb{P}_{z}\left(\zeta_{0} \leq t\right)=$ $\mathbb{E}_{\infty}\left(e^{-z U_{t}^{0}}\right)>0$. By Lemma 5.3-1), $U_{t}^{0} \underset{t \rightarrow \infty}{\longrightarrow} 0$ a.s and thus $\mathbb{P}_{z}\left(\zeta_{0} \leq t\right) \underset{t \rightarrow \infty}{\longrightarrow} \mathbb{P}_{z}\left(\zeta_{0}<\infty\right)=1$. This proves Theorem 3.9-1).

It remains to study the process when $\frac{2 \lambda}{c} \geq 1$. The next lemma gives part 2) and part 3) of Theorem 3.9.

Lemma 7.8. Assume $\frac{2 \lambda}{c} \geq 1$. 
1) If $-\Psi$ is the Laplace exponent of a subordinator then the process is absorbed at $\infty$ almost surely.

2) If $-\Psi$ is not the Laplace exponent of a subordinator then the process tends to 0 with probability given by (4.4) and on the event of extinction, the process gets absorbed at 0 if and only if $\int^{\infty} \frac{\mathrm{d} u}{\Psi(u)}<\infty$.

Proof. By Lemma 7.6, the process $\left(Z_{t}, t \geq 0\right)$ has 0 and $\infty$ as absorbing boundary. By Lemma 7.4, it satisfies (MP), therefore $\left(Z_{t}, t \geq 0\right)$ has the same law as $\left(Z_{t}^{\min }, t \geq 0\right)$.

Proof of 1). Assume that $-\Psi$ is the Laplace exponent of a subordinator, then by Lemma 4.6, explosion is almost sure. 4.3

Proof of 2). If now $-\Psi$ is not the Laplace exponent of a subordinator, then by Lemma

$$
\mathbb{P}_{z}\left(Z_{t} \underset{t \rightarrow \infty}{\longrightarrow} 0\right)=\mathbb{P}_{z}\left(\zeta_{\infty}=\infty\right)=\mathbb{P}_{z}\left(\sigma_{0}<\infty\right)>0
$$

Similarly as in the proof of Lemma 6.5, Lemma 7.3 entails that $\mathbb{P}_{z}\left(\zeta_{0} \leq t\right)=\mathbb{E}_{\infty}\left(e^{-x U_{t}}\right)$. The latter is positive if and only if $\infty$ is an entrance boundary for $\left(U_{t}, t \geq 0\right)$. Lemma 5.22 ) ensures that this is the case if and only if $\int^{\infty} \frac{\mathrm{d} z}{\Psi(z)}<\infty$. Note that when $\int^{\infty} \frac{\mathrm{d} z}{\Psi(z)}<\infty$ then $\left\{\zeta_{0}<\infty\right\}=\left\{\zeta_{\infty}=\infty\right\}=\left\{\zeta_{0}<\zeta_{\infty}\right\}$.

As mentioned in the introduction, our extension of the minimal process has been done without using excursion theory. In particular, we have not discussed the existence of a local time at $\infty$. We end this article by showing that when $\infty$ is regular reflecting, the process immediately returns to $\infty$ after leaving it. Such a boundary is said to be regular for itself and standard theory, see for instance Bertoin [Ber96, Chapter IV], would then ensure the existence of a local time.

Proposition 7.9. Assume $\mathcal{E}<\infty$ and $0 \leq \frac{2 \lambda}{c}<1$. Set $S_{\infty}:=\inf \left\{t>0 ; Z_{t}=\infty\right\}$, then $\mathbb{P}_{\infty}\left(S_{\infty}=0\right)=1$.

Proof. Assume $\mathcal{E}<\infty$. Under $\mathbb{P}_{z}$ with $z \in(0, \infty), S_{\infty}$ and $\zeta_{\infty}$ have the same law. We prove that $\mathbb{E}_{z}\left[S_{\infty}, S_{\infty}<\infty\right] \underset{z \rightarrow \infty}{\longrightarrow} 0$. Recall that by Lemma 7.4, under $\mathbb{P}_{z}$, the process $\left(Z_{t \wedge S_{\infty}}, t \geq 0\right)$ has the same law as $\left(Z_{t}^{\min }, t \geq 0\right)$ which, according to Section 4 , can be seen as a time-changed Ornstein-Uhlenbeck-type process starting from $z,\left(R_{s}^{(z)}, s \geq 0\right)$ as given in (4.2). We have that

$$
\mathbb{E}_{z}\left[S_{\infty}, S_{\infty}<\infty\right]=\mathbb{E}\left[\int_{0}^{\infty} \frac{\mathrm{d} s}{R_{s}^{(z)}}, \sigma_{0}=\infty\right]
$$

Since $\mathcal{E}<\infty, R_{s}^{(z)} \underset{s \rightarrow \infty}{\longrightarrow} \infty$ and $\inf _{s \geq 0} R_{s}^{(z)}>0$ a.s. on the event $\left\{\sigma_{0}=\infty\right\}$. From (4.2) and a direct comparison argument, we see that $\inf _{s \geq 0} R_{s}^{(z)} \geq \inf _{s \geq 0} R_{s}^{\left(z^{\prime}\right)}$ almost surely for any $z \geq z^{\prime}$.

Assume first that $-\Psi$ is not the Laplace exponent of a subordinator. Then letting $\mu$ to 0 and then $z$ to $\infty$ in (4.5) provides

$$
\mathbb{P}_{z}\left(\sigma_{a}<\infty\right)=\mathbb{P}\left(\inf _{s \geq 0} R_{s}^{(z)} \leq a\right) \underset{z \rightarrow \infty}{\longrightarrow} 0 .
$$

Therefore $\inf _{s \geq 0} R_{s}^{(z)} \underset{z \rightarrow \infty}{\longrightarrow} \infty$ almost surely. From the inequality (4.7), we see by applying Lebesgue's theorem that for any $b>0$,

$$
\mathbb{E}\left[\int_{0}^{\infty} \frac{1-e^{-b R_{s}^{(z)}}}{R_{s}^{(z)}} \mathrm{d} s, \sigma_{0}=\infty\right] \underset{z \rightarrow \infty}{\longrightarrow} 0 .
$$


Since

$$
\mathbb{E}\left[\int_{0}^{\infty} \frac{1-e^{-b R_{s}^{(z)}}}{R_{s}^{(z)}} \mathrm{d} s, \sigma_{0}=\infty\right] \geq \mathbb{E}\left[\left(1-e^{-b \inf _{s \geq 0}^{(z)} R_{s}^{(z)}}\right) \int_{0}^{\infty} \frac{\mathrm{d} s}{R_{s}^{(z)}}, \sigma_{0}=\infty\right]
$$

and $\inf _{s \geq 0} R_{s}^{(z)} \underset{z \rightarrow \infty}{\longrightarrow} \infty$ almost surely, by applying Lebesgue's theorem, we deduce that

$$
\mathbb{E}\left[\int_{0}^{\infty} \frac{\mathrm{d} s}{R_{s}^{(z)}}, \sigma_{0}=\infty\right] \underset{z \rightarrow \infty}{\longrightarrow} 0
$$

By the Markov inequality, $\mathbb{P}_{z}\left(S_{\infty}>t, S_{\infty}<\infty\right) \leq \frac{1}{t} \mathbb{E}_{z}\left[S_{\infty}, S_{\infty}<\infty\right]$ and thus

$$
\mathbb{P}_{z}\left(S_{\infty}>t, S_{\infty}<\infty\right) \underset{z \rightarrow \infty}{\longrightarrow} 0
$$

Let $s>0$. By the Markov property at time $s$,

$$
\begin{aligned}
\mathbb{E}_{\infty}\left[\mathbb{1}_{\left\{S_{\infty}>t+s\right\}} \mathbb{1}_{\left\{S_{\infty}<\infty\right\}}\right] & =\mathbb{E}_{\infty}\left[\mathbb{E}\left[\mathbb{1}_{\left\{S_{\infty}>t+s\right\}} \mathbb{1}_{\left\{S_{\infty}<\infty\right\}} \mid \mathcal{F}_{s}\right]\right] \\
& \leq \mathbb{E}_{\infty}\left[\mathbb{E}_{Z_{s}}\left[\mathbb{1}_{\left\{S_{\infty}>t\right\}} \mathbb{1}_{\left\{S_{\infty}<\infty\right\}}\right]\right]
\end{aligned}
$$

By right continuity, $Z_{s} \underset{s \rightarrow 0+}{\longrightarrow} \infty$ a.s under $\mathbb{P}_{\infty}$, and by (7.2),

$$
\mathbb{E}_{Z_{s}}\left[\mathbb{1}_{\left\{S_{\infty}>t\right\}} \mathbb{1}_{\left\{S_{\infty}<\infty\right\}}\right] \underset{s \rightarrow 0+}{\longrightarrow} 0
$$

a.s under $\mathbb{P}_{\infty}$. By Lebesgue's theorem, we conclude that $\mathbb{P}_{\infty}\left(S_{\infty}>t, S_{\infty}<\infty\right)=0$ for any $t>0$.

We now verify that $\mathbb{P}_{\infty}\left(S_{\infty}=\infty\right)=0$. One has $\left\{S_{\infty}=\infty\right\}=\left\{\sigma_{0}<\infty\right\}$ under $\mathbb{P}_{z}$ for any $z \in[0, \infty)$. By the Markov property,

$$
\mathbb{P}_{\infty}\left(S_{\infty}=\infty\right)=\mathbb{E}_{\infty}\left(\mathbb{E}\left(\mathbb{1}_{\left\{S_{\infty}=\infty\right\}} \mid \mathcal{F}_{s}\right)\right)=\mathbb{E}_{\infty}\left(\mathbb{P}_{Z_{s}}\left(S_{\infty}=\infty\right)\right)
$$

We see in Lemma 4.3 that $\mathbb{P}_{z}\left(\sigma_{0}<\infty\right) \underset{z \rightarrow \infty}{\longrightarrow} 0$. Since $Z_{s} \underset{s \rightarrow 0}{\longrightarrow} \infty \mathbb{P}_{\infty}$-a.s, we have that $\mathbb{P}_{\infty}\left(S_{\infty}=\infty\right)=0$. This entails that for any $t>0$,

$$
\mathbb{P}_{\infty}\left(S_{\infty}>t, S_{\infty}<\infty\right)=\mathbb{P}_{\infty}\left(S_{\infty}>t\right)=0
$$

and thus $\mathbb{P}_{\infty}\left(S_{\infty}=0\right)=1$.

Assume now that $-\Psi$ is the Laplace exponent of a subordinator and $\delta \geq 0$ is its drift. One may argue by comparison as follows. Let $b>\delta$ and $\tilde{\Psi}(q):=\Psi(q)+b q$. Set $\left(\tilde{R}_{s}^{(z)}, s \geq 0\right)$ the Ornstein-Uhlenbeck-type process associated with the Lévy process $\tilde{Y}_{t}=Y_{t}-b t$. The function $-\tilde{\Psi}$ is not the Laplace exponent of a subordinator and one has almost surely for any $s$ and any $z, R_{s}^{(z)} \geq \tilde{R}_{s}^{(z)}$. Applying the previous arguments to $\left(\tilde{R}_{s}^{(z)}, s \geq 0\right)$ will provide $\mathbb{P}_{\infty}\left(S_{\infty}=0\right)=1$.

The last proposition leads to the natural question of characterizing the inverse local time. A related question is to see if one can continue Table 1 to the case where the boundary $\infty$ of $Z$ is regular absorbing. This requires us to work with the reflected diffusion and seems to demand a deeper analysis of the duality at the level of generators. 


\section{Appendix}

Proof of Proposition 3.13. Recall

$$
\mathcal{E}=\int_{0}^{\theta} \frac{1}{x} \exp \left(\frac{2}{c} \int_{x}^{\theta} \frac{\Psi(u)}{u} \mathrm{~d} u\right) \mathrm{d} x \text { and } \mathcal{E}^{\prime}=\int_{0}^{\theta} \frac{1}{x} \exp \left(-\frac{2}{c} \int_{1}^{\infty} e^{-x v} \frac{\bar{\pi}(v)}{v} \mathrm{~d} v\right) \mathrm{d} x .
$$

From the Lévy-Khintchine form (2.3) of $\Psi$, one has for any $u>0$,

$$
\frac{\Psi(u)}{u}=-\int_{0}^{\infty} e^{-u v} \bar{\pi}(v \vee 1) \mathrm{d} v+\gamma+\frac{\sigma^{2}}{2} u+\int_{0}^{1} \pi((v, 1))\left(1-e^{-u v}\right) \mathrm{d} v
$$

where $v \vee 1=\max (v, 1)$. For any $z>0$, set $H(z):=-\int_{z}^{\theta} \frac{\Psi(u)}{u} \mathrm{~d} u$. One can readily check from the above expression that as $z$ goes to 0 ,

$$
H(z)=\int_{1}^{\infty} e^{-z v} \frac{\bar{\pi}(v)}{v} \mathrm{~d} v+\epsilon_{\theta}(z)+c_{\theta}
$$

for some constant $c_{\theta}$ and some function $\epsilon_{\theta}$ such that $\epsilon_{\theta}(z) \underset{z \rightarrow 0}{\longrightarrow} 0$. Observe first that $\int_{0} \frac{[\Psi(u) \mid}{u} \mathrm{~d} u<\infty$ if and only if $\int_{1}^{\infty} \frac{\bar{\pi}(v)}{v} \mathrm{~d} v<\infty$. Tonelli's theorem ensures that it is equivalent to $\int^{\infty} \log (v) \pi(\mathrm{d} v)<\infty$. Since the term $\epsilon_{\theta}(z)+c_{\theta}$ will not play any role in the convergence of $\mathcal{E}, \mathcal{E}$ and $\mathcal{E}^{\prime}$ have the same nature. Without loss of generality, we assume $\pi((0,1))=0, \sigma=0$ and $\gamma=0$. Define $\Phi$ such that $\Phi(z) / z=H(z)$. The function $\Phi$ is the Laplace exponent of a driftless subordinator with Lévy measure $\nu$ whose tail is $\bar{\nu}(v)=\frac{\bar{\pi}(v)}{v}$ for all $v \geq 1$. Recall from [Ber96, Chapter III, Proposition 1] that there exists a universal constant $\kappa>0$ such that

$$
\frac{1}{\kappa} I(1 / z) \leq \Phi(z) / z \leq \kappa I(1 / z), \forall z>0, \text { where } I(z):=\int_{1}^{z} \bar{\nu}(r) \mathrm{d} r .
$$

Thus, we have

$$
\frac{1}{\kappa} \int_{1}^{1 / z} \frac{\bar{\pi}(u)}{u} \mathrm{~d} u \leq H(z) \leq \kappa \int_{1}^{1 / z} \frac{\bar{\pi}(u)}{u} \mathrm{~d} u
$$

Therefore

$$
\int_{0}^{\theta} \frac{1}{x} \exp \left(-\frac{2 \kappa}{c} \int_{1}^{1 / x} \frac{\bar{\pi}(u)}{u} \mathrm{~d} u\right) \mathrm{d} x \leq \mathcal{E} \leq \int_{0}^{\theta} \frac{1}{x} \exp \left(-\frac{2}{c \kappa} \int_{1}^{1 / x} \frac{\bar{\pi}(u)}{u} \mathrm{~d} u\right) \mathrm{d} x .
$$

We now study the boundaries of $\left(U_{t}, t<\tau\right)$ solution to (5.1) and establish the results stated in Section 5. This is a regular ${ }^{3}$ diffusion in $(0, \infty)$. We apply Feller's tests for which we refer to Durrett's book [Dur96, Chapter 6, Section 6.2 and 6.5]. Denote by $s$ the scale function of $\left(U_{t}, t<\tau\right)$ and by $m$ the density of its speed measure. Fix $\theta \in(0, \infty)$. For any $x \in(0, \infty)$,

$$
s(x)=\int_{\theta}^{x} \exp \left(\int_{\theta}^{y} \frac{2 \Psi(u)}{c u} \mathrm{~d} u\right) \mathrm{d} y, \quad m(x)=\frac{2}{c x} \exp \left(-\int_{\theta}^{x} \frac{2 \Psi(u)}{c u} \mathrm{~d} u\right) .
$$

Denote by $M$ the antiderivative of $m, M(z)=\int_{\theta}^{z} m(x) \mathrm{d} x$. We establish Lemma 5.2 and Lemma 5.3. Recall the basic analytical properties of the function $\Psi$ mentioned in Section 2.

\footnotetext{
${ }^{3}$ in the sense irreducible
} 
Proof of Lemma 5.2-2. Feller's tests imply that $\infty$ is inaccessible if and only if

$$
I_{\infty}=\int_{\theta}^{\infty}(s(\infty)-s(x)) m(x) \mathrm{d} x=\int_{\theta}^{\infty} \frac{2 \mathrm{~d} x}{c x} \int_{x}^{\infty} \exp \left(\int_{x}^{y} \frac{2 \Psi(u)}{c u} \mathrm{~d} u\right) \mathrm{d} y=\infty
$$

and an entrance boundary if and only if $I_{\infty}=\infty$ and

$$
J_{\infty}=\int_{\theta}^{\infty}(M(\infty)-M(z)) s^{\prime}(z) \mathrm{d} z=\int_{\theta}^{\infty} \mathrm{d} z \int_{z}^{\infty} \frac{2}{c x} \exp \left(-\int_{z}^{x} \frac{2 \Psi(u)}{c u} \mathrm{~d} u\right) \mathrm{d} x<\infty .
$$

If $-\Psi$ is not the Laplace exponent of a subordinator then there exists $z_{0} \geq 0$, such that for all $u \geq z_{0}, \Psi(u) \geq 0$ and then $I_{\infty}=\infty$. If $-\Psi$ is the Laplace exponent of a subordinator then $\lim _{z \rightarrow \infty} \Psi(z) / z=: \mathbf{d}<0$. Therefore, for any $\epsilon>0$, there exists $z_{0}$ such that if $u \geq z_{0}$

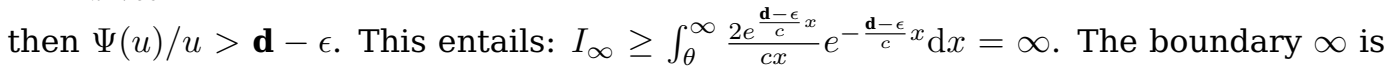
therefore always inaccessible.

We show now that $J_{\infty}<\infty$ if and only if $\int^{\infty} \frac{\mathrm{d} u}{|\Psi(u)|}<\infty$. Define $Q(x):=\int_{\theta}^{x} \frac{2 \Psi(u)}{c u} \mathrm{~d} u$, one has

$$
J_{\infty}=\int_{\theta}^{\infty} e^{Q(z)} \int_{z}^{\infty} \frac{2 e^{-Q(x)}}{c x} \mathrm{~d} x \mathrm{~d} z .
$$

Plainly if $-\Psi$ is the Laplace exponent of a subordinator, $Q(x) \leq 0$ for all $x$ and $J_{\infty}=\infty$. We focus on the non-subordinator case, in which $\Psi(z)>0$ for large enough $z$. Assume $\int^{\infty} \frac{\mathrm{d} z}{\Psi(z)}<\infty$. Note that $\Psi$ is non-decreasing over $(\rho, \infty)$ where $\rho$ is the largest zero of $\Psi$. Moreover, since $\int^{\infty} \frac{\mathrm{d} z}{\Psi(z)}<\infty$, there exists $v$ such that $\frac{\Psi(v)}{v} \geq 1$. Since any mechanism $\Psi$ is convex and $\Psi(0) \leq 0, z \mapsto \Psi(z) / z$ is non-decreasing, therefore for all $z \geq v, \frac{\Psi(z)}{z} \geq 1$ and $Q(\infty)=\infty$. For all $z \geq \theta>\rho$, by the mean value theorem there exists $\xi_{z} \geq z$ such that

$$
\int_{z}^{\infty} \frac{2 \Psi(x)}{c x} e^{-Q(x)} \frac{\mathrm{d} x}{\Psi(x)}=\frac{1}{\Psi\left(\xi_{z}\right)}\left[-e^{-Q(x)}\right]_{x=z}^{x=\infty}=\frac{1}{\Psi\left(\xi_{z}\right)} e^{-Q(z)} .
$$

Thus

$$
J_{\infty}=\int_{\theta}^{\infty} e^{Q(z)} \int_{z}^{\infty} \frac{2}{c x} e^{-Q(x)} \mathrm{d} x \mathrm{~d} z \leq \int_{\theta}^{\infty} \frac{\mathrm{d} z}{\Psi(z)}<\infty .
$$

Conversely, assume $J_{\infty}<\infty$. Let $\varphi(z)=M(\infty)-M(z)=\int_{z}^{\infty} \frac{2}{c x} e^{-Q(x)} \mathrm{d} x$, one has $J_{\infty}=\lim _{b \rightarrow \infty} \int_{\theta}^{b} \varphi(z) e^{Q(z)} \mathrm{d} z$. By integration by parts:

$$
\int_{\theta}^{b} \varphi(z) e^{Q(z)} \mathrm{d} z=\int_{\theta}^{b} \frac{\varphi(z)}{Q^{\prime}(z)} e^{Q(z)} Q^{\prime}(z) \mathrm{d} z=\left[\frac{\varphi(z)}{Q^{\prime}(z)} e^{Q(z)}\right]_{\theta}^{b}-\int_{\theta}^{b} e^{Q(z)}\left(\frac{\varphi(z)}{Q^{\prime}(z)}\right)^{\prime} \mathrm{d} z .
$$

Moreover,

$$
\begin{aligned}
\left(\frac{\varphi(z)}{Q^{\prime}(z)}\right)^{\prime}=\frac{\varphi^{\prime}(z) Q^{\prime}(z)-\varphi(z) Q^{\prime \prime}(z)}{Q^{\prime}(z)^{2}} & =\frac{-\frac{2}{c z} e^{-Q(z)} \frac{2 \Psi(z)}{c z}-\varphi(z)\left(\frac{2 \Psi(z)}{c z}\right)^{\prime}}{\left(\frac{2 \Psi(z)}{c z}\right)^{2}} \\
& =-\frac{e^{-Q(z)}}{\Psi(z)}-\frac{\varphi(z)\left(\frac{2 \Psi(z)}{c z}\right)^{\prime}}{\left(\frac{2 \Psi(z)}{c z}\right)^{2}} \leq-\frac{e^{-Q(z)}}{\Psi(z)} .
\end{aligned}
$$

The last inequality holds since $\left(\frac{\Psi(z)}{z}\right)^{\prime} \geq 0$ and entails

$$
\int_{\theta}^{b} \varphi(z) e^{Q(z)} \mathrm{d} z \geq\left[\frac{\varphi(z)}{Q^{\prime}(z)} e^{Q(z)}\right]_{\theta}^{b}+\int_{\theta}^{b} \frac{\mathrm{d} z}{\Psi(z)} .
$$


We show now that $\varphi(z) e^{Q(z)} \underset{z \rightarrow \infty}{\longrightarrow} 0$. Let $\epsilon>0$ and choose $a$ large enough such that $\Psi(u) \geq \Psi(a) \geq \frac{1}{\epsilon}$ for any $u \geq z \geq a$. One has $-\int_{z}^{x} \frac{2}{c} \frac{\Psi(u)}{u} \mathrm{~d} u \leq-2 \frac{\Psi(a)}{c} \ln (x / z)$ and thus

$$
\begin{aligned}
\varphi(z) e^{Q(z)} & =\int_{z}^{\infty} \frac{2}{c x} e^{-\int_{z}^{x} \frac{2 \Psi(u)}{c u} \mathrm{~d} u} \mathrm{~d} x \\
& \leq \frac{2}{c} z^{\frac{2 \Psi(a)}{c}} \int_{z}^{\infty} x^{-2 \Psi(a) / c-1} \mathrm{~d} x \\
& =\frac{1}{\Psi(a)} \leq \epsilon .
\end{aligned}
$$

Provided that $\lim _{z \rightarrow \infty} Q^{\prime}(z) \neq 0$, (7.5) gives

$$
\int_{\theta}^{\infty} \frac{\mathrm{d} z}{\Psi(z)} \leq J_{\infty}+\frac{\varphi(\theta)}{Q^{\prime}(\theta)} e^{Q(\theta)}<\infty
$$

and the proof is complete. We show now that if $J_{\infty}<\infty$ then $\lim _{z \rightarrow \infty} Q^{\prime}(z) \neq 0$. Assume by contradiction that for any $\epsilon>0$, there exists $z_{0}$ such that $Q^{\prime}(z)=\frac{2 \Psi(z)}{c z} \leq \epsilon$ for all $z \geq z_{0}$. Recall $J_{\infty}=\int_{\theta}^{\infty} \mathrm{d} z \int_{z}^{\infty} \frac{2}{c x} \exp \left(-\int_{z}^{x} \frac{2 \Psi(u)}{c u} \mathrm{~d} u\right) \mathrm{d} x$, we get

$$
\begin{aligned}
J_{\infty} & \geq \frac{2}{c} \int_{z_{0}}^{\infty} \mathrm{d} z \int_{z}^{\infty} \frac{1}{x} e^{-\int_{z}^{x} \epsilon \mathrm{d} u} \mathrm{~d} x=\frac{2}{c} \int_{z_{0}}^{\infty} e^{\epsilon z} \mathrm{~d} z \int_{z}^{\infty} \frac{1}{x} e^{-\epsilon x} \mathrm{~d} x \\
& =\frac{2}{c} \int_{z_{0}}^{\infty} \mathrm{d} x\left(\int_{z_{0}}^{x} \mathrm{~d} z e^{\epsilon z}\right) \frac{e^{-\epsilon x}}{x}=\frac{2}{c} \int_{z_{0}}^{\infty} \frac{e^{\epsilon x}-e^{\epsilon z_{0}}}{\epsilon} \frac{e^{-\epsilon x}}{x} \mathrm{~d} x \\
& =\frac{2}{c} \int_{z_{0}}^{\infty} \frac{1-e^{\epsilon\left(z_{0}-x\right)}}{\epsilon x} \mathrm{~d} x=\infty .
\end{aligned}
$$

Proof of Lemma 5.2-1. As in the previous Lemma, we apply Feller's tests to study the nature of the boundary 0 . The boundary 0 is accessible if and only if

$$
I_{0}:=\int_{0}^{\theta}(s(x)-s(0)) m(x) \mathrm{d} x<\infty
$$

and the process can get out from the boundary 0 if and only if

$$
J_{0}:=\int_{0}(M(z)-M(0)) s^{\prime}(z) \mathrm{d} z<\infty
$$

where $M(z)=\int^{z} m(x) \mathrm{d} x$. The boundary 0 is thus an exit boundary (respectively a regular boundary) if $I_{0}<\infty$ and $J_{0}=\infty$ (respectively $J_{0}<\infty$ ). If $I_{0}=\infty$ and $J_{0}<\infty$ then 0 is an entrance boundary. We first show that $I_{0}<\infty$ if and only if $0 \leq \frac{2 \lambda}{c}<1$. This will provide that 0 is accessible when $0 \leq \frac{2 \lambda}{c}<1$. Since $\Psi(0)=-\lambda$ and $\Psi$ is continuous at $0+,-\frac{2}{c} \Psi(z) \underset{z \rightarrow 0+}{\longrightarrow} \frac{2 \lambda}{c}$. Assume $\frac{2 \lambda}{c}<1$, and let $\epsilon$ such that $\frac{2 \lambda}{c}+\epsilon<1$, there exists $\theta>0$ such that $-\frac{2}{c} \Psi(z) \leq \frac{2 \lambda}{c}+\epsilon<1$ for all $z \leq \theta$. Therefore,

$$
\begin{aligned}
|s(0)| & =\int_{0}^{\theta} \exp \left(-\int_{y}^{\theta} \frac{2 \Psi(z)}{c z} \mathrm{~d} z\right) \mathrm{d} y \\
& \leq \int_{0}^{\theta} \exp \left(\int_{y}^{\theta} \frac{\frac{2 \lambda}{c}+\epsilon}{z} \mathrm{~d} z\right) \mathrm{d} y \\
& =c_{\theta} \int_{0}^{\theta} \frac{\mathrm{d} y}{y^{\frac{2 \lambda}{c}+\epsilon}}<\infty .
\end{aligned}
$$


We have

$$
\begin{aligned}
I_{0} & =\frac{2}{c} \int_{0}^{\theta} \frac{\mathrm{d} x}{x} \int_{0}^{x} \exp \left(-\int_{y}^{x} \frac{2 \Psi(z)}{c z} \mathrm{~d} z\right) \mathrm{d} y \\
& \leq \frac{2}{c} \int_{0}^{\theta} \frac{\mathrm{d} x}{x} \int_{0}^{x} \exp \left(\int_{y}^{x} \frac{2 \lambda / c+\epsilon}{z} \mathrm{~d} z\right) \mathrm{d} y \\
& =\frac{1}{c} \int_{0}^{\theta} \frac{\mathrm{d} x}{x} \int_{0}^{x} \exp [(2 \lambda / c+\epsilon)(\ln (x)-\ln (y))] \mathrm{d} y \\
& =\frac{2}{c} \int_{0}^{\theta} x^{\frac{2 \lambda}{c}+\epsilon-1} \mathrm{~d} x \int_{0}^{x} \frac{1}{y^{\frac{2 \lambda}{c}+\epsilon}} \mathrm{d} y=\frac{2 \theta}{c(1-(2 \lambda / c+\epsilon))}<\infty .
\end{aligned}
$$

Therefore when $0 \leq \frac{2 \lambda}{c}<1$ for all $y \in \mathbb{R}_{+}$s.t. $y \neq x, \mathbb{P}_{x}\left(\tau_{0}<\tau_{y}\right)>0$, where $\tau_{y}$ is the first hitting time of $y$ by the diffusion $\left(U_{t}, t \geq 0\right)$. Assume $\frac{2 \lambda}{c} \geq 1$ and define $\tilde{\Psi}(z)=\lambda+\Psi(z)$. Note that $\tilde{\Psi}$ is the mechanism of a CSBP with no killing term. One has

$$
|s(0)|=\int_{0}^{\theta} \frac{1}{y^{2 \lambda / c}} \exp \left(-\int_{y}^{\theta} \frac{2 \tilde{\Psi}(z)}{c z} \mathrm{~d} z\right) \mathrm{d} y .
$$

If $\tilde{\Psi}(z) \geq 0$ for all $z \geq 0$, then $\lim _{z \rightarrow 0} \frac{\tilde{\Psi}(z)}{z}=\tilde{\Psi}^{\prime}(0) \geq 0$ and $\int_{y}^{\theta} \frac{2 \tilde{\Psi}(z)}{c z} \mathrm{~d} z \underset{y \rightarrow 0}{\longrightarrow} \int_{0}^{\theta} \frac{2 \tilde{\Psi}(z)}{c z} \mathrm{~d} z<\infty$ thus $|s(0)|=\infty$. If $\tilde{\Psi}$ is non-positive in a neighbourhood of 0 then $|s(0)| \geq \int_{0}^{\theta} \frac{\mathrm{d} y}{y^{2 \lambda / c}}=\infty$, which implies that 0 is inaccessible.

We study now $J_{0}$. When $0 \leq \frac{2 \lambda}{c}<1,|s(0)|<\infty$ and $J_{0}$ has the same nature as $|M(0)|=\frac{c}{2} \mathcal{E}$. We deduce that 0 is an exit if $\mathcal{E}=\infty$, regular if $0 \leq \frac{2 \lambda}{c}<1$ and $\mathcal{E}<\infty$. Assume $\frac{2 \lambda}{c} \geq 1$. One has

$$
\begin{aligned}
J_{0} & =\int_{0}^{\theta} \mathrm{d} z \int_{0}^{z} \frac{2}{c x} \exp \left(\int_{x}^{z} \frac{2 \Psi(u)}{c u} \mathrm{~d} u\right) \mathrm{d} x=\frac{2}{c} \int_{0}^{\theta} \mathrm{d} z \int_{0}^{z} \frac{1}{x} e^{-\frac{2 \lambda}{c} \int_{x}^{z} \frac{d u}{u}} e^{\int_{x}^{z} \frac{2 \tilde{\Psi}(u)}{c u} \mathrm{~d} u} \mathrm{~d} x \\
& =\frac{2}{c} \int_{0}^{\theta} \frac{\mathrm{d} z}{z^{2 \lambda / c}} \int_{0}^{z} x^{2 \lambda / c-1} e^{\int_{x}^{z} \frac{2 \tilde{\Psi}(u)}{c u} \mathrm{~d} u} \mathrm{~d} x
\end{aligned}
$$

If $\tilde{\Psi}$ is non-positive in a neighbourhood of 0 , then

$$
J_{0} \leq \frac{2}{c} \int_{0}^{\theta} \frac{\mathrm{d} z}{z^{2 \lambda / c}} \int_{0}^{z} x^{2 \lambda / c-1} \mathrm{~d} x=\frac{\theta}{\lambda}<\infty .
$$

If $\tilde{\Psi}(z) \geq 0$ for all $z \geq 0$, then $\lim _{z \rightarrow 0} \tilde{\Psi}(z) / z \geq 0$ and then $0 \leq \int_{x}^{z} \frac{2 \tilde{\Psi}(u)}{c u} \mathrm{~d} u \leq \int_{0}^{\theta} \frac{2 \tilde{\Psi}(u)}{c u} \mathrm{~d} u<\infty$, which provides $J_{0}<\infty$. We deduce that if $\frac{2 \lambda}{c} \geq 1$, then 0 is an entrance.

Recall that $\left(U_{t}^{0}, t \geq 0\right)$ denotes the $\Psi$-generalized Feller diffusion with 0 as an exit (if $\mathcal{E}=\infty$ ) or a regular absorbing boundary (if $\mathcal{E}<\infty$ and $\frac{2 \lambda}{c}<1$ ).

Proof of Lemma 5.3-1. By see [Dur96, Theorem 3.3 page 220], if $s(\infty)=\infty$ then $\tau_{\infty}=\infty$ a.s. By assumption $-\Psi$ is not the Laplace exponent of a subordinator, we see from (7.3) that this entails $\mathbf{d}=\liminf _{z \rightarrow \infty} \frac{\Psi(z)}{z}>0$. One has

$$
s(\infty)=\int^{\infty} \exp \left(\int_{\theta}^{y} \frac{2 \Psi(z)}{c z} \mathrm{~d} z\right) \mathrm{d} y \geq \int^{\infty} \exp \left(\frac{2 \mathbf{d}}{c} y\right) \mathrm{d} y=\infty .
$$

Thus, $\mathbb{P}_{x}\left(\tau_{0}<\tau_{\infty}\right)=1$ for all $x \geq 0$. 
Recall that if $\Psi(z) \leq 0$ for all $z \geq 0$, then $\Psi$ is of the form (7):

$$
\Psi(v)=-\lambda-\delta v-\int_{0}^{\infty}\left(1-e^{-v u}\right) \pi(\mathrm{d} u)
$$

with $\delta \geq 0$ and $\int_{0}^{\infty}(1 \wedge u) \pi(\mathrm{d} u)<\infty$. In this case, $-\Psi$ is the Laplace exponent of a subordinator. Recall the condition $(\mathbf{A})$ :

$$
\delta=0 \text { and } \bar{\pi}(0)+\lambda \leq c / 2 .
$$

Proof of Lemma 5.3-2). We show i) and ii) simultaneously. We have seen in (7.6) that $|s(0)|<\infty$. If $s(\infty)<\infty$, then [Dur96, Theorem 3.3 page 220] ensures

$$
\mathbb{P}_{x}\left(U_{t}^{0} \underset{t \rightarrow+\infty}{\longrightarrow} 0\right)=\frac{s(\infty)-s(x)}{s(\infty)-s(0)} .
$$

Moreover if $s(\infty)=\infty$ then $\mathbb{P}_{x}\left(U_{t}^{0} \underset{t \rightarrow+\infty}{\longrightarrow} 0\right)=1$. We show that $s(\infty)=\infty$ if and only if $(A)$ is satisfied. One has $s(\infty)=\int_{\theta}^{\infty} \exp \left(\int_{\theta}^{y} \frac{2 \Psi(z)}{c z} \mathrm{~d} z\right) \mathrm{d} y$. Note that $\frac{\Psi(z)}{z}=$ $-\frac{\lambda}{z}-\delta-\int_{0}^{\infty} e^{-z x} \bar{\pi}(x) \mathrm{d} x$. We adapt the proof of Lambert [Lam05, Section 4.1]. We first discuss the case of (A) not satisfied. This corresponds to assume that at least one of the following conditions is satisfied:

$$
\delta>0 \text { or } \delta=0, \bar{\pi}(0)<+\infty \text { and } \bar{\pi}(0)+\lambda>c / 2 \text { or } \delta=0 \text { and } \bar{\pi}(0)=+\infty .
$$

- Assume $\delta>0$. Since $\frac{\Psi(z)}{z} \underset{z \rightarrow+\infty}{\longrightarrow}-\delta$, there exists $y_{0}$ such that for all $z \geq y_{0}$, $\frac{2 \Psi(z)}{c z} \leq-\frac{2 \delta}{c}+\frac{\delta}{c}=-\frac{\delta}{c}<0$. Therefore

$$
\begin{aligned}
s(\infty)=\int_{\theta}^{\infty} \exp \left(\int_{\theta}^{y_{0}} \frac{2 \Psi(z)}{c z} \mathrm{~d} z+\int_{y_{0}}^{y} \frac{2 \Psi(z)}{c z} \mathrm{~d} z\right) & \leq c_{y_{0}} \int_{\theta}^{\infty} \exp \left(-\int_{y_{0}}^{y} \frac{\delta}{c} \mathrm{~d} z\right) \mathrm{d} y \\
& =c_{y_{0}} e^{\frac{\delta}{c} y_{0}} \int_{\theta}^{\infty} e^{-\frac{\delta}{c} y} \mathrm{~d} y<\infty
\end{aligned}
$$

where $c_{y_{0}}=\exp \left(\int_{\theta}^{y_{0}} \frac{2 \Psi(z)}{c z} \mathrm{~d} z\right)$.

- Assume $\delta=0, \bar{\pi}(0)<+\infty$ and $\bar{\pi}(0)+\lambda>c / 2$. By Tonelli, one has

$$
\begin{aligned}
-\int_{\theta}^{y} \frac{2 \Psi(z)}{c z} \mathrm{~d} z & =\frac{2 \lambda}{c} \ln \left(\frac{y}{\theta}\right)+\frac{2}{c} \int_{\theta}^{y} \mathrm{~d} z\left(\int_{0}^{\infty} e^{-z x} \bar{\pi}(x) \mathrm{d} x\right) \\
& =\frac{2 \lambda}{c} \ln \left(\frac{y}{\theta}\right)+\frac{2}{c} \int_{0}^{\infty} \frac{e^{-\theta x}-e^{-y x}}{x} \bar{\pi}(x) \mathrm{d} x .
\end{aligned}
$$

We will show that under the assumption $\bar{\pi}(0)<\infty$,

$$
k_{\theta}(x):=\lim _{y \rightarrow \infty}\left[\int_{0}^{\infty} \frac{e^{-\theta z}-e^{-y z}}{z} \bar{\pi}(z) \mathrm{d} z-\int_{x / y}^{x} e^{-\theta z} \frac{\bar{\pi}(z)}{z} \mathrm{~d} z\right] \text { exists and is finite. }
$$

Assume for now that (7.10) is proven. Since $\int_{x / y}^{x} e^{-\theta z} \frac{\bar{\pi}(z)}{z} \mathrm{~d} z \geq \bar{\pi}(x) e^{-\theta x} \ln (y)$, there exists $y_{0}$ such that if $y \geq y_{0}$, then

$$
\begin{aligned}
-\frac{2}{c} \int_{\theta}^{y} \frac{\Psi(z)}{z} \mathrm{~d} z & \geq \frac{2}{c}\left(k_{\theta}(x)-1-\lambda \ln (\theta)+\lambda \ln (y)+\int_{x / y}^{x} e^{-\theta z} \frac{\bar{\pi}(z)}{z} \mathrm{~d} z\right) \\
& \geq c_{\theta}(x)+\frac{2}{c}\left(\lambda+\bar{\pi}(x) e^{-\theta x}\right) \ln y .
\end{aligned}
$$


Choose $x$ close enough to 0 such that $\lambda+\bar{\pi}(x) e^{-\theta x}>c / 2$. Therefore

$$
\begin{aligned}
s(\infty) & =\int_{\theta}^{\infty} \exp \left(\int_{\theta}^{y} \frac{2 \Psi(z)}{c z} \mathrm{~d} z\right) \mathrm{d} y \\
& \leq K_{\theta, y_{0}}+\int_{y_{0}}^{\infty} \exp \left(c_{\theta}(x)-\frac{2}{c}\left(\lambda+\bar{\pi}(x) e^{-\theta x}\right) \ln y\right) \mathrm{d} y \\
& =K_{\theta, y_{0}}+K_{\theta, x} \int_{\theta}^{\infty} y^{-\frac{2}{c}\left(\lambda+\bar{\pi}(x) e^{-\theta x}\right)} \mathrm{d} y<\infty
\end{aligned}
$$

where $K_{\theta, y_{0}}$ and $K_{\theta, x}$ are some constants.

- Assume that $\bar{\pi}(0)=+\infty$ and define $\pi_{b}(\mathrm{~d} x)$ by $\bar{\pi}_{b}(x)=\bar{\pi}(x \vee b)$. For all $b>0$ and all $x \geq 0, \bar{\pi}_{b}(x) \leq \bar{\pi}(x)$. The measure $\pi_{b}$ is finite and one can choose $b$ such that $\bar{\pi}_{b}(0)>c$. One has for $y$ large enough:

$$
\int_{0}^{\infty} \frac{e^{-\theta x}-e^{-y x}}{x} \bar{\pi}(x) \mathrm{d} x \geq \int_{0}^{\infty} \frac{e^{-\theta x}-e^{-y x}}{x} \bar{\pi}_{b}(x) \mathrm{d} x
$$

Applying the same argument as above with $\lambda=0$ and $\pi_{b}$ instead of $\pi$, one obtains $s(\infty)<\infty$.

Assume now that condition (A) holds. Namely, $\delta=0, \bar{\pi}(0)<+\infty$ and $\bar{\pi}(0)+\lambda \leq c / 2$. By using (7.10), there exists $y_{0}$ such that for $y \geq y_{0}$,

$$
\begin{aligned}
-\int_{\theta}^{y} \frac{2 \Psi(z)}{c z} \mathrm{~d} z & \leq k_{\theta}(x)+1+\frac{2 \lambda}{c} \ln \left(\frac{y}{\theta}\right)+\frac{2 \lambda}{c} \int_{x / y}^{x} e^{-\theta z} \frac{\bar{\pi}(z)}{z} \mathrm{~d} z \\
& \leq k_{\theta}(x)+1-\frac{2 \lambda}{c} \ln (\theta)+\frac{2}{c}(\lambda+\bar{\pi}(0)) \ln (y) .
\end{aligned}
$$

Since $\bar{\pi}(0)+\lambda \leq c / 2$,

$$
s(\infty) \geq K_{\theta, y_{0}}+K_{\theta, x} \int_{\theta}^{\infty} y^{-2(\bar{\pi}(0)+\lambda) / c} \mathrm{~d} y=\infty .
$$

We show now (7.10):

$$
\begin{aligned}
& \int_{0}^{\infty}\left(e^{-\theta z}-e^{-y z}\right) \frac{\bar{\pi}(z)}{z} \mathrm{~d} z-\int_{x / y}^{x} e^{-\theta z} \frac{\bar{\pi}(z)}{z} \mathrm{~d} z \\
& =\int_{0}^{x / y}\left(e^{-\theta z}-e^{-y z}\right) \frac{\bar{\pi}(z)}{z} \mathrm{~d} z-\int_{x / y}^{x} e^{-y z} \frac{\bar{\pi}(z)}{z} \mathrm{~d} z+\int_{x}^{\infty}\left(e^{-\theta z}-e^{-y z}\right) \frac{\bar{\pi}(z)}{z} \mathrm{~d} z \\
& =\underbrace{\int_{0}^{x / y}\left(e^{-\theta z}-e^{-y z}\right) \frac{\bar{\pi}(z)}{z} \mathrm{~d} z}_{I_{1}(x, y)}-\underbrace{\int_{x}^{x y} e^{-u} \frac{\bar{\pi}(u / y)}{u} \mathrm{~d} u}_{I_{2}(x, y)}+\underbrace{\int_{x}^{\infty}\left(e^{-\theta z}-e^{-y z}\right) \frac{\bar{\pi}(z)}{z} \mathrm{~d} z}_{I_{3}(x, y)} .
\end{aligned}
$$

By changing variable, one has $I_{1}(x, y)=\int_{0}^{x}\left(e^{-\theta \frac{u}{y}}-e^{-u}\right) \frac{\bar{\pi}(u / y)}{u} \mathrm{~d} u$. Since $y \mapsto \bar{\pi}(u / y)$ is non-decreasing and converges to $\bar{\pi}(0)$ as $y$ goes to $\infty$, one can apply the monotone convergence theorem, this provides

$$
I_{1}(x, y) \underset{y \rightarrow+\infty}{\longrightarrow} \bar{\pi}(0) \int_{0}^{x}\left(1-e^{-u}\right) \frac{\mathrm{d} u}{u}<\infty .
$$

The monotone convergence theorem readily applies to $I_{2}(x, y)$ :

$$
I_{2}(x, y) \underset{y \rightarrow+\infty}{\longrightarrow} \bar{\pi}(0) \int_{x}^{\infty} \frac{e^{-u}}{u} \mathrm{~d} u<\infty .
$$


By Lebesgue's theorem, $I_{3}(x, y)=\int_{x}^{\infty}\left(e^{-\theta z}-e^{-y z}\right) \frac{\bar{\pi}(z)}{z} \mathrm{~d} z \underset{y \rightarrow \infty}{\longrightarrow} \int_{x}^{\infty} \frac{e^{-\theta z} \bar{\pi}(z)}{z} \mathrm{~d} z<\infty$. Finally,

$$
k_{\theta}(x)=\bar{\pi}(0)\left(\int_{0}^{x} \frac{1-e^{-u}}{u} \mathrm{~d} u+\int_{x}^{\infty} \frac{e^{-u}}{u} \mathrm{~d} u\right)+\int_{x}^{\infty} e^{-\theta z} \frac{\bar{\pi}(z)}{z} \mathrm{~d} z<\infty .
$$

As formula (4.5) for the Laplace transform of the first entrance times of OrnsteinUhlenbeck-type processes with positive jumps has played an important role in our study, we end this appendix by establishing it. We refer the reader to Patie [Pat05] in the context of Ornstein-Uhlenbeck process with negative jumps. Recall $\left(R_{t}, t \geq 0\right)$ the Ornstein-Uhlenbeck type process with parameters $\Psi$ and $c / 2$ as introduced in Section 4 . For any $a \geq 0$, recall $\sigma_{a}:=\inf \left\{t \geq 0, R_{t} \leq a\right\}$.

Proof of formula (4.5). For any $\mu \geq 0$, recall $g_{\mu}(x)=x^{\frac{2 \mu}{c}-1} e^{-\int_{\theta}^{x} \frac{x \Psi(y)}{c y} \mathrm{~d} y}$. The function $g_{\mu}$ solves the following equation

$$
\left(\Psi(x)-\mu+\frac{c}{2}\right) g_{\mu}(x)+\frac{c}{2} x g_{\mu}^{\prime}(x)=0 \text { for all } x \geq 0 .
$$

We have seen in the proof of Lemma 4.3 that $\int_{b}^{\infty} g_{\mu}(x) \mathrm{d} x<\infty$ for any $b>0$ and any $\mu \geq 0$. We check now that if $\mu>0$ then $\int_{0}^{b} g_{\mu}(x) \mathrm{d} x<\infty$. Note that either $\int_{0}^{\theta} \frac{\Psi(u)}{u} \mathrm{~d} u=-\infty$ or $\int_{0}^{\theta} \frac{\Psi(u)}{u} \mathrm{~d} u \in(-\infty, \infty)$. In both cases, for any $b>0$ there is a constant $C>0$ such that $\int_{0}^{b} g_{\mu}(x) \mathrm{d} x \leq C \int_{0}^{b} x^{\frac{2 \mu}{c}-1} \mathrm{~d} x<\infty$ since $\mu>0$. Thus, we have $\int_{0}^{\infty} g_{\mu}(x) \mathrm{d} x<\infty$ for any $\mu>0$.

Set $f_{\mu}(z):=\int_{0}^{\infty} e^{-x z} g_{\mu}(x) \mathrm{d} x$ for any $z \geq 0$. This is a $C_{0}^{2}$ decreasing function. For any $z \in(0, \infty)$ and $x \in(0, \infty), \mathcal{L}^{R} e_{x}(z)=\Psi(x) e_{x}(z)+\frac{c}{2} x z e_{x}(z)$. We now verify that $f_{\mu}$ is an eigenfunction of the generator $\mathcal{L}^{R}$ :

$$
\begin{aligned}
& \mathcal{L}^{R} f_{\mu}(z)-\mu f_{\mu}(z) \\
& =\int_{0}^{\infty} g_{\mu}(x)\left(\mathcal{L}^{R} e_{x}(z)-\mu e_{x}(z)\right) \mathrm{d} x \\
& =\int_{0}^{\infty}(\Psi(x)-\mu) e^{-x z} g_{\mu}(x) \mathrm{d} x+\int_{0}^{\infty} \frac{c}{2} z e^{-x z} x g_{\mu}(x) \mathrm{d} x \\
& =\int_{0}^{\infty}(\Psi(x)-\mu) e^{-x z} g_{\mu}(x) \mathrm{d} x+\frac{c}{2}\left(\left[-e^{-x z} x g_{\mu}(x)\right]_{x=0}^{x=\infty}+\int_{0}^{\infty} e^{-x z}\left(g_{\mu}(x)+x g_{\mu}^{\prime}(x)\right) \mathrm{d} x\right) \\
& =\int_{0}^{\infty}\left(\left(\Psi(x)-\mu+\frac{c}{2}\right) g_{\mu}(x)+\frac{c}{2} x g_{\mu}^{\prime}(x)\right) e^{-x z} \mathrm{~d} x=0 .
\end{aligned}
$$

Itô's formula entails that the process $\left(e^{-\mu t} f_{\mu}\left(R_{t}\right), t \geq 0\right)$ is a local martingale. Since $\left(R_{t}, t \geq 0\right)$ has no negative jumps, and the function $f_{\mu}$ is decreasing, one has for any $t \leq \sigma_{a}, R_{t} \geq a$ and $f_{\mu}\left(R_{t}\right) \leq f_{\mu}(a) \mathbb{P}_{z}$-a.s, for all $z \geq a$. Therefore, the process $\left(e^{-\mu\left(t \wedge \sigma_{a}\right)} f_{\mu}\left(R_{t \wedge \sigma_{a}}\right), t \geq 0\right)$ is a bounded martingale, and by the optional stopping theorem, one gets

$$
\mathbb{E}_{z}\left[e^{-\mu \sigma_{a}}\right]=\frac{f_{\mu}(z)}{f_{\mu}(a)}
$$

Acknowledgments. I would like to thank Gerónimo Uribe-Bravo for a precious help in the proof of Lemma 6.1, Adrian Gonzales-Casanova for helpful discussions, as well as anonymous referees for thorough reports. This work is partially supported by the French National Research Agency (ANR): ANR GRAAL (ANR-14-CE25-0014) and by LABEX MME-DII (ANR11-LBX-0023-01). 


\section{References}

[BP15] Mamadou Ba and Etienne Pardoux, Branching processes with interaction and a generalized Ray-Knight theorem, Ann. Inst. H. Poincaré Probab. Statist. 51 (2015), no. 4, 1290-1313.

[Ber04] Julien Berestycki, Exchangeable fragmentation-coalescence processes and their equilibrium measures, Electron. J. Probab. 9 (2004), no. 25, 770-824. MR-2110018

[BFF17] J. Berestycki, M. C. Fittipaldi, and J. Fontbona, Ray-Knight representation of flows of branching processes with competition by pruning of Lévy trees, Probability Theory and Related Fields (2017).

[Ber96] Jean Bertoin, Lévy processes, Cambridge Tracts in Mathematics, vol. 121, Cambridge University Press, Cambridge, 1996. MR-1406564

[BK16] Jean Bertoin and Igor Kortchemski, Self-similar scaling limits of Markov chains on the positive integers, Ann. Appl. Probab. 26 (2016), no. 4, 2556-2595. MR-3543905

[CLUB09] Ma. Emilia Caballero, Amaury Lambert, and Gerónimo Uribe Bravo, Proof(s) of the Lamperti representation of continuous-state branching processes, Probab. Surv. 6 (2009), 62-89. MR-2592395

$\left[\mathrm{CCL}^{+}\right.$09] Patrick Cattiaux, Pierre Collet, Amaury Lambert, Servet Martinez, Sylvie Méléard, and Jaime San Martín, Quasi-stationary distributions and diffusion models in population dynamics, Ann. Probab. 37 (2009), no. 5, 1926-1969.

[CR84] J. Theodore Cox and Uwe Rösler, A duality relation for entrance and exit laws for Markov processes, Stochastic Process. Appl. 16 (1984), no. 2, 141-156. MR-724061

[Don91] Peter Donnelly, Weak convergence to a Markov chain with an entrance boundary: ancestral processes in population genetics, Ann. Probab. 19 (1991), no. 3, 1102-1117. MR-1112408

[Dur96] Richard Durrett, Stochastic calculus, Probability and Stochastics Series, CRC Press, Boca Raton, FL, 1996, A practical introduction. MR-1398879

[EK86] Stewart N. Ethier and Thomas G. Kurtz, Markov processes, Wiley Series in Probability and Mathematical Statistics: Probability and Mathematical Statistics, John Wiley \& Sons Inc., New York, 1986, Characterization and convergence. MR-838085

[Fel54] William Feller, Diffusion processes in one dimension, Trans. Amer. Math. Soc. 77 (1954), 1-31. MR-0063607

[Gre74] D. R. Grey, Asymptotic behaviour of continuous time, continuous state-space branching processes, J. Appl. Probability 11 (1974), 669-677. MR-0408016

[Gri74] Anders Grimvall, On the convergence of sequences of branching processes, Ann. Probability 2 (1974), 1027-1045. MR-0362529

[Had85] Dimitar I. Hadjiev, The first passage problem for generalized Ornstein-Uhlenbeck processes with nonpositive jumps, Séminaire de probabilités, XIX, 1983/84, Lecture Notes in Math., vol. 1123, Springer, Berlin, 1985, pp. 80-90. MR-889469

[HT04] Paul Horridge and Roger Tribe, On stationary distributions for the KPP equation with branching noise, Ann. Inst. H. Poincaré Probab. Statist. 40 (2004), no. 6, 759-770. MR-2096217

[HW07] M. Hutzenthaler and A. Wakolbinger, Ergodic behavior of locally regulated branching populations, Ann. Appl. Probab. 17 (2007), no. 2, 474-501. MR-2308333

[Jiř58] Miloslav Jiřina, Stochastic branching processes with continuous state space, Czechoslovak Math. J. 8 (83) (1958), 292-313. MR-0101554

[Kal02] Olav Kallenberg, Foundations of modern probability, second ed., Probability and its Applications (New York), Springer-Verlag, New York, 2002. MR-1876169

[Kyp14] Andreas E. Kyprianou, Fluctuations of Lévy processes with applications, second ed., Universitext, Springer, Heidelberg, 2014, Introductory lectures. MR-3155252

[KPRS17] Andreas E. Kyprianou, Steven W. Pagett, Tim Rogers, and Jason Schweinsberg, A phase transition in excursions from infinity of the fast fragmentation-coalescence process, Ann. Probab. 45 (2017), no. 6A, 3829-3849. 
Logistic CSBPs: duality and reflection at infinity

[Lam67a] John Lamperti, Continuous state branching processes, Bull. Amer. Math. Soc. 73 (1967), 382-386. MR-0208685

[Lam67b] John Lamperti, The limit of a sequence of branching processes, Z. Wahrscheinlichkeitstheorie und Verw. Gebiete 7 (1967), 271-288. MR-0217893

[Lam05] Amaury Lambert, The branching process with logistic growth, Ann. Appl. Probab. 15 (2005), no. 2, 1506-1535. MR-2134113

[LPW13] V. Le, E. Pardoux, and A. Wakolbinger, "Trees under attack": a Ray-Knight representation of Feller's branching diffusion with logistic growth, Probab. Theory Related Fields 155 (2013), no. 3-4, 583-619. MR-3034788

[Li18] Pei-Sen Li, A continuous-state polynomial branching process, Stochastic Processes and their Applications (2018).

[Lig05] Thomas M. Liggett, Interacting particle systems, Classics in Mathematics, SpringerVerlag, Berlin, 2005, Reprint of the 1985 original. MR-2108619

[Par16] Étienne Pardoux, Probabilistic models of population evolution, Mathematical Biosciences Institute Lecture Series. Stochastics in Biological Systems, vol. 1, Springer, [Cham]; MBI Mathematical Biosciences Institute, Ohio State University, Columbus, $\mathrm{OH}, 2016$, Scaling limits, genealogies and interactions. MR-3496029

[Pat05] Pierre Patie, On a martingale associated to generalized Ornstein-Uhlenbeck processes and an application to finance, Stochastic Process. Appl. 115 (2005), no. 4, 593-607. MR-2128632

[RY99] Daniel Revuz and Marc Yor, Continuous martingales and Brownian motion, third ed., Grundlehren der Mathematischen Wissenschaften [Fundamental Principles of Mathematical Sciences], vol. 293, Springer-Verlag, Berlin, 1999. MR-1725357

[Sat13] Ken-iti Sato, Lévy processes and infinitely divisible distributions, Cambridge Studies in Advanced Mathematics, vol. 68, Cambridge University Press, Cambridge, 2013, Translated from the 1990 Japanese original, Revised edition of the 1999 English translation. MR-3185174

[SY84] Ken-iti Sato and Makoto Yamazato, Operator-self-decomposable distributions as limit distributions of processes of Ornstein-Uhlenbeck type, Stochastic Process. Appl. 17 (1984), no. 1, 73-100. MR-738769

[Shi90] Tokuzo Shiga, A recurrence criterion for Markov processes of Ornstein-Uhlenbeck type, Probab. Theory Related Fields 85 (1990), no. 4, 425-447. MR-1061937

[Sil68] M. L. Silverstein, A new approach to local times, J. Math. Mech. 17 (1967/1968), 1023-1054. MR-0226734 